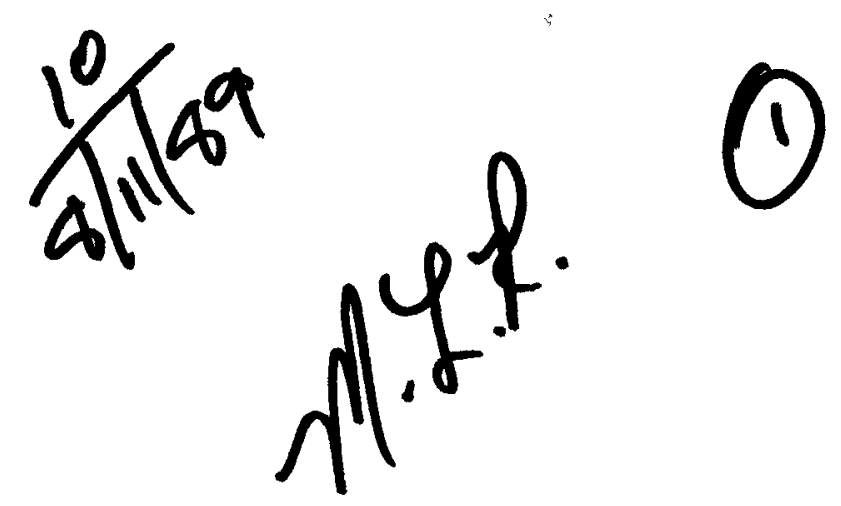

UCID-21720

\title{
The Underground Electromagnetic Pulse: Four Representative Models
}

\author{
L. F. Wouters
}

\section{June 1989}

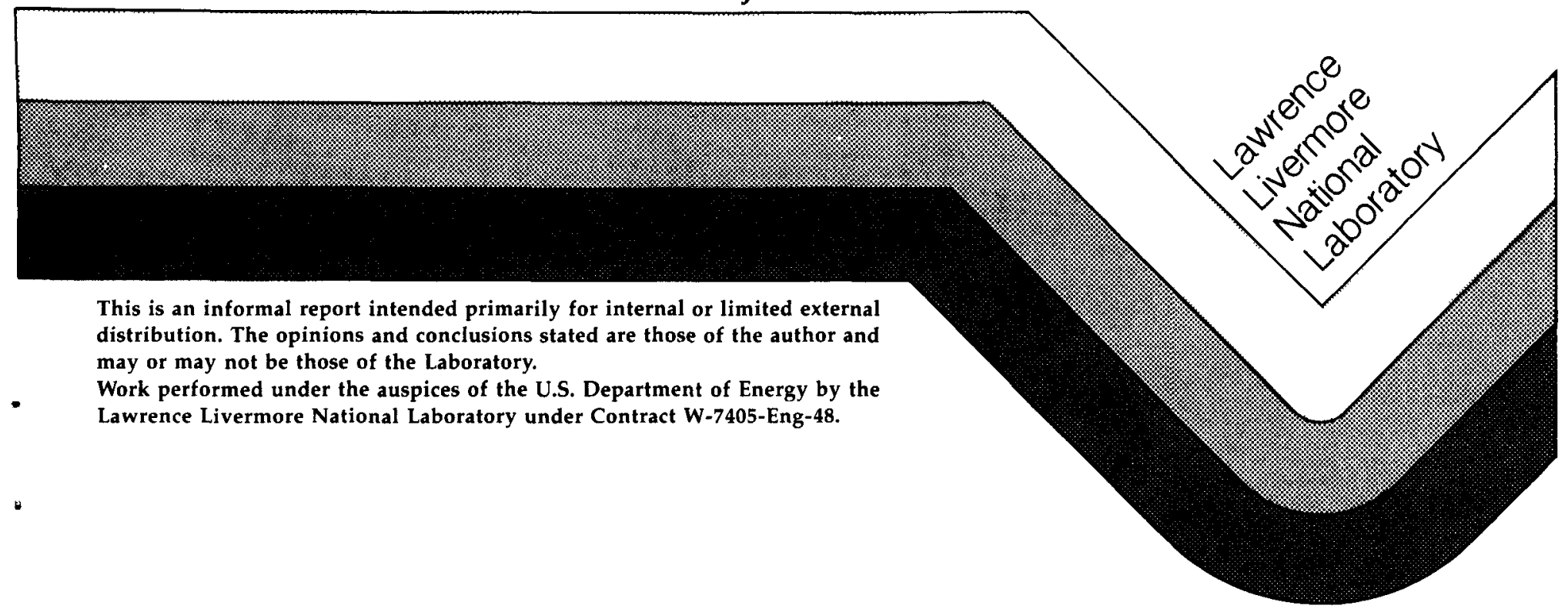

This is an informal report intended primarily for internal or limited external distribution. The opinions and conclusions stated are those of the author and may or may not be those of the Laboratory.

Work performed under the auspices of the U.S. Department of Energy by the

Lawrence Livermore National Laboratory under Contract W-7405-Eng-48. 


\section{DISCLAIMER}

This report was prepared as an account of work sponsored by an agency of the United States Government. Neither the United States Government nor any agency Thereof, nor any of their employees, makes any warranty, express or implied, or assumes any legal liability or responsibility for the accuracy, completeness, or usefulness of any information, apparatus, product, or process disclosed, or represents that its use would not infringe privately owned rights. Reference herein to any specific commercial product, process, or service by trade name, trademark, manufacturer, or otherwise does not necessarily constitute or imply its endorsement, recommendation, or favoring by the United States Government or any agency thereof. The views and opinions of authors expressed herein do not necessarily state or reflect those of the United States Government or any agency thereof. 


\section{DISCLAIMER}

Portions of this document may be illegible in electronic image products. Images are produced from the best available original document. 


\section{DISCLAIMER}

This document was prepared as an account of work sponsored by an agency of the United States Government. Neither the United States Government nor the University of California nor any of their employees, makes any warranty, express or implied, or assumes any legal liability or responsibility for the accuracy, completeness, or usefulness of any information, apparatus, product, or process disclosed, or represents that its use would not infringe privately owned rights. Reference herein to any specific commercial products, process, or service by trade name, trademark, manufacturer, or otherwise, does not necessarily constitute or imply its endorsement, recommendation, or favoring by the United States Government or the University of California. The views and opinions of authors expressed herein do not necessarily state or reflect those of the United States Government or the University of California, and shall not be used for advertising or product endorsement purposes.

\footnotetext{
This report has been reproduced directly from the best available copy.

Available to DOE and DOE contractors from the Office of Scientific and Technical Information P.O. Box 62, Oak Ridge, TN 37831

Prices available from (615) 576-8401, FTS 626-8401.

Available to the public from the National Technical Information Service

U.S. Department of Commerce 5285 Port Royal Rd., Springfield, VA 22161
}

Price

Code

A01

Papercopy Prices

A02

A03

A04

A05

A06

A07

A08

A09
Page

Range

Microfiche

001-050

051-100

101-200

201-300

301-400

401-500

501-600

601

\section{DO NOT MICRiFiLL" THIS PAUEE}




\section{Contents}

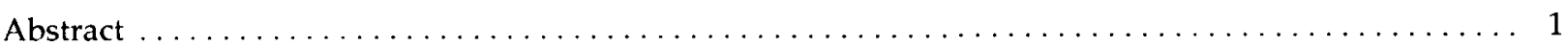

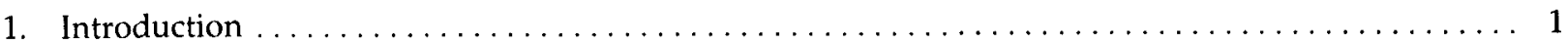

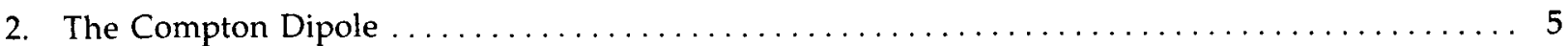

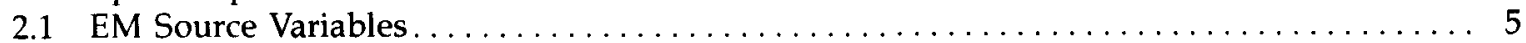

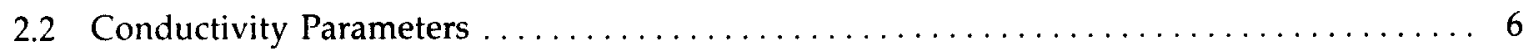

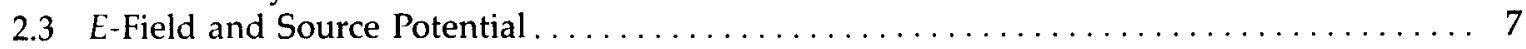

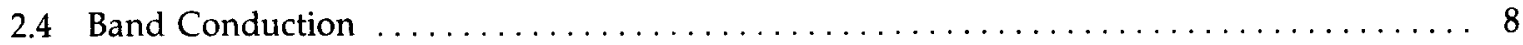

2.5 Compton-Current Dipole Moment $\ldots \ldots \ldots \ldots \ldots \ldots \ldots \ldots \ldots \ldots \ldots \ldots \ldots \ldots \ldots$

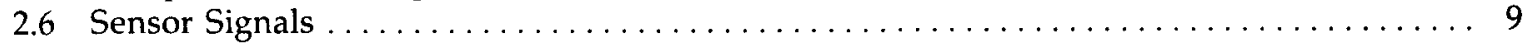

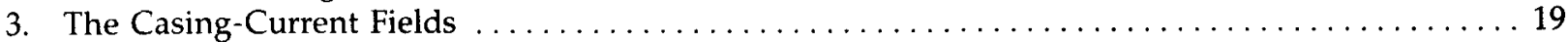

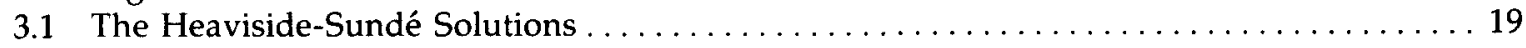

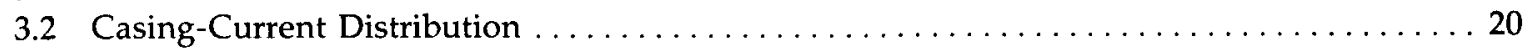

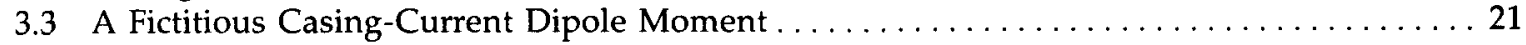

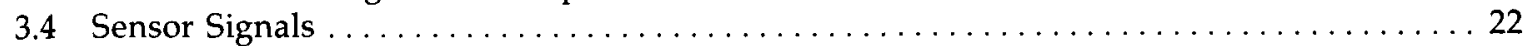

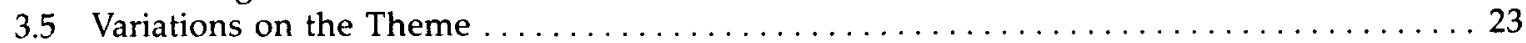

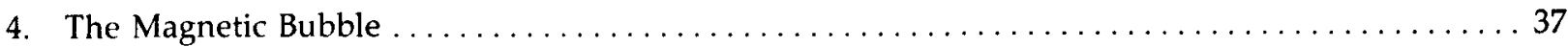

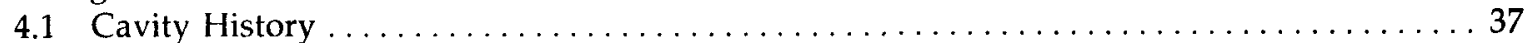

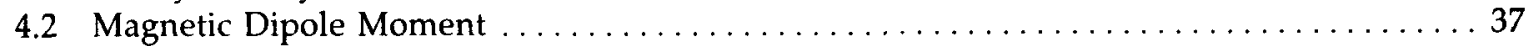

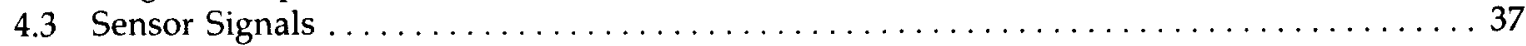

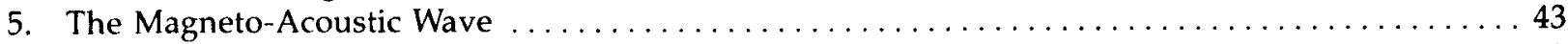

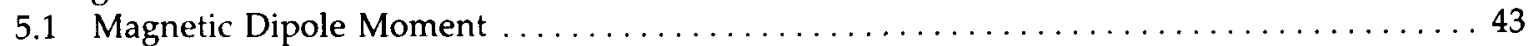

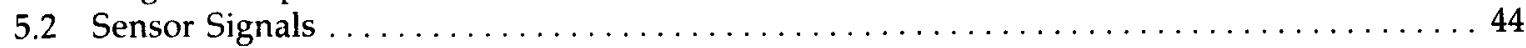

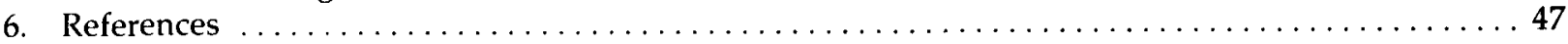

Appendix A-Compton-Dipole IFTs (Local Case Only) $\ldots \ldots \ldots \ldots \ldots \ldots \ldots \ldots \ldots \ldots \ldots \ldots 48$

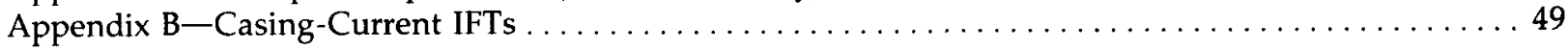

\section{DISCLAIMER}

This report was prepared as an account of work sponsored by an agency of the United States Government. Neither the United States Government nor any agency thereof, nor any of their employees, makes any warranty, express or implied, or assumes any legal liability or responsibility for the accuracy, completeness, or usefulness of any information, apparatus, product, or process disclosed, or represents that its use would not infringe privately owned rights. Reference herein to any specific commercial product, process, or service by trade name, trademark, manufacturer, or otherwise does not necessarily constitute or imply its endorsement, recommendation, or favoring by the United States Government or any agency thereof. The views and opinions of authors expressed herein do not necessarily state or reflect those of the United States Government or any agency thereof. 


\title{
The Underground Electromagnetic Pulse: Four Representative Models
}

\begin{abstract}
I describe four phenomenological models by which an underground nuclear explosion may generate electromagnetic pulses:

- Compton current asymmetry (or "Compton dipole").

- Uphole conductor currents (or "casing currents").

- Diamagnetic cavity plasma (or "magnetic bubble").

- Large-scale ground motion (or "magneto-acoustic wave").

I outline the corresponding analytic exercises and summarize the principal results of the computations. I used a 10-kt contained explosion as the fiducial case. Each analytic sequence developed an equivalent source dipole and calculated signal waveforms at representative ground-surface locations.

As a comparative summary, the Compton dipole generates a peak source current moment of about $12,000 \mathrm{~A} \cdot \mathrm{m}$ in the submicrosecond time domain. The casing-current source model obtains an equivalent peak moment of about $2 \times 10^{5} \mathrm{~A} \cdot \mathrm{m}$ in the 10 - to 30 $\mu \mathrm{s}$ domain. The magnetic bubble produces a magnetic dipole moment of about $7 \times 10^{6} \mathrm{~A} \cdot \mathrm{m}^{2}$, characterized by a $30-\mathrm{ms}$ time structure. Finally, the magneto-acoustic wave corresponds to a magnetic dipole moment of about $600 \mathrm{~A} \cdot \mathrm{m}^{2}$, with a waveform showing 0.5 -s periodicities.
\end{abstract}

\section{Introduction}

This report presents the results of certain calculations of the electromagnetic pulse (EMP) generated by a nominal-yield nuclear explosion in a contained underground test. It addresses four basic source mechanisms, as depicted in Fig. 1-1. They include two magneto-dynamic processes:

- Expansion of the conductive-cavity "bubble" in the earth's magnetic field.

- Faraday currents induced by the subsequent ground motion beyond cavity formation.

Also, two Compton-current-driven processes:

- Local current-dipole at the test canister itself.

- Surface currents subsequently induced on the uphole conductors.

The exemplary fiducial case for quantitatively rating these models is a fictitious $10-\mathrm{kt}$ test, as depicted and specified in Fig. 1-2. Its hydrodynamic features correspond to the MERLIN nuclear test, which was extensively instrumented and evaluated by a group with Sandia National Laboratories, Albuquerque (SNIA). ${ }^{1}$ Its prompt-nuclear features correspond to the generic model described by Glasstone. ${ }^{2}$

The calculations obtained the histories of the $E$ and $H$ fields for each model, at ground surface and at two representative ranges: "Local" $(r=d=200 \mathrm{~m})$ and "Distant" $(r=5 \mathrm{~km})$. As we will see, the predicted signals at $5 \mathrm{~km}$ are very small (undetectable, in some cases), such that there appears to be no purpose in considering much greater distances. A fundamental entity throughout these considerations is the Von Hippel (VH) frequency, expressed as $\omega_{\mathrm{c}}=\left(\mu_{0} \sigma_{\mathrm{g}} d^{2} / 2\right)^{-1}$. For the earth conductivities addressed here $\left(\sigma_{\mathrm{g}}\right)$, $\omega_{\mathrm{c}}$ has the values shown in Table 1-1.

In each model, the source-zone of interaction turns out to be small compared to its principal electromagnetic (EM) wavelength. That permitted a conventional two-staged calculational approach. For each case, I derived an equivalent small-dipole source representation, which I used as input to the well-known formalisms that describe the EM fields from such dipoles in conducting media. ${ }^{3,4}$ In all cases, the significant frequency regime is such that $\kappa \kappa_{0} \omega \ll \sigma_{\mathrm{g}}$ (the earth conductivity); hence, the radiative term is always small-to-vanishing. Its suppression permits considerable computational simplification. 
The frequency domain for the magneto-dynamic models is very low $(\omega<300 \mathrm{rad} / \mathrm{s})$. Hence, the EM attenuations and dispersions along the paths to the fiducial surface-sensor locations are very small; the time-history of an observed EM field closely reproduces that of its source dipole. In contrast, the frequencies for the Compton-driven models range upward to $\sim 100 \mathrm{MHz}$, so that the dispersions (or phase shifts) through the earth medium can be very large. For these, I adopted a Fourier formalism:

Current history $\rightarrow$ Frequency resolution $\rightarrow$ Path transmission $\rightarrow$ Time-domain reconstitution.

At the Local sensor range, both the direct earth path and the "up-and-over" path may contribute to the observed signal. At a Distant sensor, only the up-and-over path is significant; the observed EM fields derive from the current distribution induced just below ground surface around "surtace greund aro" (Sc;/), the top of the emplacement hole. The Compton-driven cases are cylindrically symmetric around the vertical emplacement-hole axis. In contrast, the magnetodynamic cases have a tilted axis of symmetry parallel to the Earth's magnetic field $\left(H_{\mathrm{e}}\right)$ so that the surface fields would exhibit orientational effects (N-S, E-W).

Table 1-1. Earth conductivities and corresponding Von Hippel frequencies.

\begin{tabular}{|cccc|}
\hline$\sigma_{\mathrm{g}}(\mathrm{mho} / \mathrm{m})$ & $0.0025^{*}$ & 0.005 & 0.01 \\
$\omega_{\mathrm{c}}(\mathrm{rad} / \mathrm{s})$ & 16,000 & 8000 & 4000
\end{tabular}

${ }^{*} \sigma_{\mathrm{B}}=0.0025$ was used as the fiducial example value. 


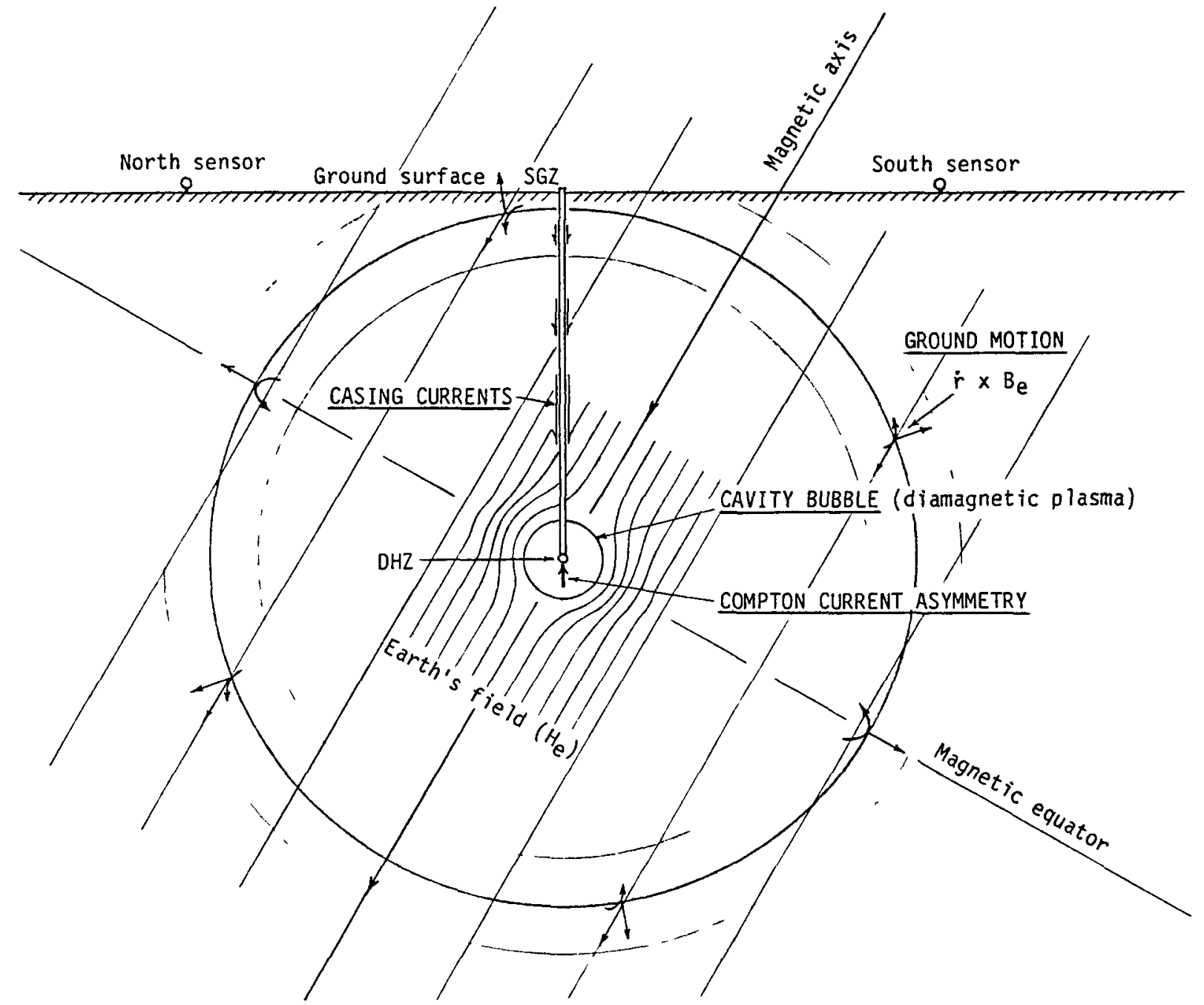

Figure 1-1. Four UG EMP source mechanisms and related features. 


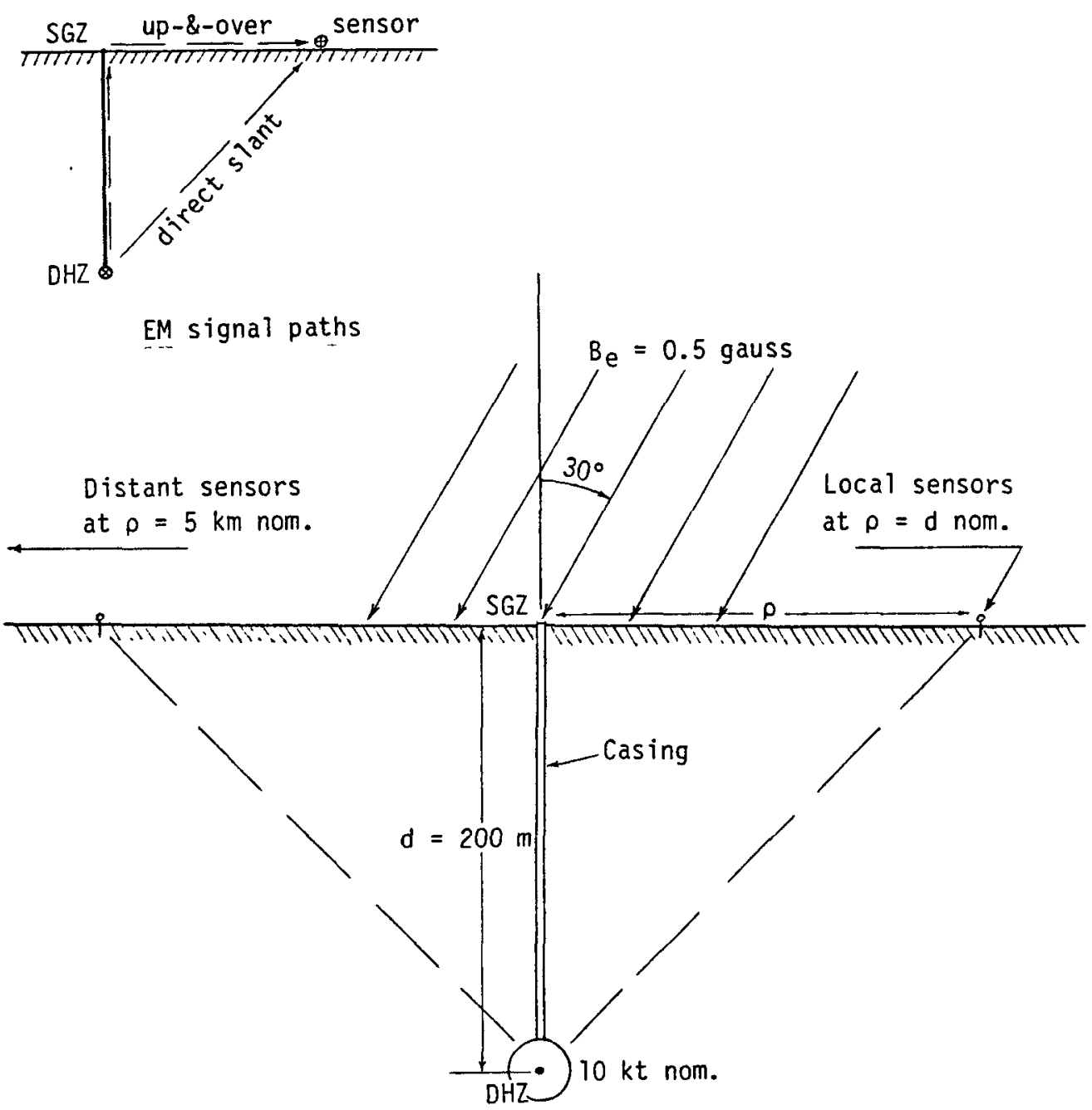

Figure 1-2. The fiducial nuclear test. 


\section{The Compton Dipole}

The appropriate driver for the Compton-current models is the radial potential induced by the gamma-generated Compton-electron flux through the earth medium immediately surrounding the test canister. I calculated it as the integral of the induced radial $E$-field; this determines the available source currents for both the Compton-dipole and casing-current mechanisms." The most critical feature is the specification of the induced earth conductivity due to Compton ionization. As in the classical case of atmospheric EMP, that leads to a conduction counter-current that severely limits ("saturates") the induced $E$-field.

\subsection{EM Source Variables}

For this fiducial exercise, I characterized the underground test geometry as a hollow sphere enclosing a centrally located point gamma source, as depicted in Fig. 2-1. (The asymmetry introduced by the uphole test structure will be subsequently addressed as a perturbation.) The Glasstone gamma-source history, shown in Fig. 2-2, was fitted analytically by $\Phi_{\gamma}=\Phi_{\mathrm{b}} f(t)$, where

$f(t)=\left\{\begin{array}{lll}e^{\alpha t} & \text { for } t<0 & \text { (alpha phase) } \\ 4 R\left(e^{-b t}-e^{-2 b t}\right)+1 & \text { for } 0<t<T_{\mathrm{d}} & \text { (peak phase) } \\ e^{-\gamma t^{\prime}} & \text { for } T_{d}<t & \text { (decay phase) }\end{array}\right\}$.

$\Phi_{\mathrm{b}}$ characterizes the gamma output at the alpha-to-peak transition (or "break-point") corresponding to $t=0$, as this time scale is defined. In the calculations, the fiducial case used these coefficient values:

$$
\begin{aligned}
& \alpha=10^{8} \mathrm{~s}^{-1} \\
& R=\Phi_{\mathrm{c}} / \Phi_{\mathrm{b}}=20 \\
& \gamma=2 \times 10^{7} \mathrm{~s}^{-1}
\end{aligned}
$$

$$
\begin{aligned}
& \Phi_{\mathrm{d}} \approx \Phi_{\mathrm{b}}=10^{29} \mathrm{\gamma MeV} / \mathrm{s} \\
& \Phi_{\mathrm{c}} \approx 2 \times 10^{30} \mathrm{MeV} / \mathrm{s} \\
& T_{\mathrm{d}}=4 \times 10^{-8} \mathrm{~s}
\end{aligned}
$$

These numbers (and Fig. 2-2) correspond to a direct scaling of the Glasstone gamma-source depiction (at $1 \mathrm{kt}$ ) to $10 \mathrm{kt}$ (see Ref. 2, §1 1.54 , pp. 16 and 17, and Fig. 8-14, p. 328). For the purpose of illustration, I also adjusted the breakpoint to be about equal to the peak-to-decay transition at $T_{\mathrm{d}}{ }^{\dagger}$. The character " $b$ " is not further defined, as it was intrinsically set by specifying $T_{\mathrm{d}}$ in the computations. Furthermore, these particular details of the peak phase turn out to be inconsequential, as one result of the extremely severe saturation-truncation of the E-field.

The local gamma flux at a distance $r$ (into the surrounding earth) is

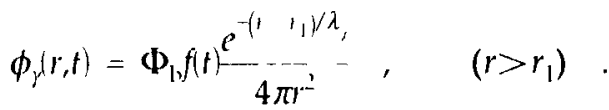

For typical alluvial test media, $\lambda_{\gamma}=15 \mathrm{~cm}$. ${ }^{\ddagger}$ The fiducial problem used $r_{1}=60 \mathrm{~cm}=4 \lambda_{\gamma}$ as the idealized test-cavity radius.

The Maxwell-significant part of the Compton flux produced in the earth is the "forward" radially projected current component. I expressed it as the current density: $J_{c}=j \phi_{\gamma}(t)$, where the conversion coefficient is $j=\left(e / \varepsilon_{\gamma}\right)\left(\lambda_{\beta} / \lambda_{\gamma}\right) \approx 10^{-21} \mathrm{mks}$. The conversion efficiency is $\lambda_{\beta} / \lambda_{\gamma}=0.02$; this number is relatively

\footnotetext{
* At first glance, the driver would appear to be the Compton current ejected from the canister into the earth, but the net current associated with the radial potential is about an order of magnitude greater.

' $t$ ' expresses a sutably shifted time base.

† The mean gamma-photon energy was taken as $\varepsilon_{\gamma}=3.2 \mathrm{MeV}$. The reason for such a high-value choice is that the principal $E_{r}$ contributions occur relatively far into the earth $(\sim 8 \mathrm{mfp})$, where the gamma flux has become fairly hard.
} 
insensitive to $Z$ and $\varepsilon_{\gamma}$ For orientation, consider the situation at the entrance radius $r_{1}$ when $t=0$ (break point): $\phi_{\gamma}=\phi_{\mathrm{b}}=2.2 \times 10^{28} \gamma \mathrm{MeV} / \mathrm{m}^{2} \mathrm{~s}, J_{\mathrm{c}}=2.2 \times 10^{7} \mathrm{~A} / \mathrm{m}^{2}$. It is already an intense situation; there's still $\times 20$ left to go. Obviously, the electromagnetic properties of the gamma-illuminated earth medium will have been altered well before this condition is reached.*

The spherical symmetry reduces the Maxwell equations to one expression:

$\kappa \kappa_{0} \partial E_{r} / \partial t+\left(U_{\mathrm{c}}+\sigma E_{r}\right)=0$.

$U_{c}=J_{c}(r, t), \sigma=\sigma(r, t)$, of course.] This was solved iteratively together with the coupled integral:

$E_{r}=\int\left(\partial E_{r} / \partial t\right) d t$

A third integration obtains the instantaneous radial potential:

$V_{r}(t)=\int_{r_{1}}^{r_{2}} E_{r}(t) d r$

where the outer radius $r_{2}$ is set by a low residual gamma flux threshold. ${ }^{+}$

This representation assumes that the asymmetries associated with the two extended-field mechanisms do not significantly degrade it. Putting it pragmatically, the Compton-dipole and casing-current elements present impedances (to $V_{r}$ ) that are high compared to the intrinsic source impedance of the $\phi_{\gamma}-E_{r}$ sphere.

For considerations of $\sigma$ at high $\phi_{\gamma}$ levels, the test medium is hardly an ideal substance; alluvium might best be described as globally amorphous, microcrystalline and partly electrolytic. Both kinetic and band conduction can contribute to $\sigma E$. (Ionic transport is too slow.) Above, the specification of the driving current $J_{\mathrm{c}}$ as a linear function of $\phi_{\gamma}$ was relatively straightforward. $\sigma$ does not yield so easily; it is radically nonlinear with respect to $\phi_{\gamma}$.

From velocity measurements, $v=c / \sqrt{\kappa}=10^{8} \mathrm{~m} / \mathrm{s}$ so that $\kappa=9 . \ddagger$

\subsection{Conductivity Parameters}

The conventional theory expresses mobile charge-carrier conductivity as $\sigma=N e \mu$. For the fiducial examples, I restricted the modeling of $\sigma$ to a "single mean" carrier species, consisting of quasi-free electrons. These can exist in two states: as epithermal particles transported kinetically through the atomic matrix by the induced $E$-field, and as conduction-band occupants. The carrier density $(\mathrm{Ne})$ is determined through a competition between production by Compton-electron ionization $(\dot{q})$ and a variety of capture processes that immobilize the carrier electrons. To first order, $\dot{q}$ is also proportional to $\phi_{\gamma}$, written as ${ }^{\S}$

$\dot{q}=\left(\frac{\phi_{\gamma}}{\varepsilon_{\gamma}}\right)\left(\frac{1 \partial \varepsilon}{\varepsilon_{\mathrm{p}} \partial x}\right)=\xi \phi_{\mathrm{b}} f(t)$

For the alluvial test medium, the ionization coefficient has the value: $\xi=1.4 \times 10^{5} \mathrm{~m}^{-1}$.

To account for carrier removal, I used the conventional formalism to determine the mobile-carrier density $\left(N_{\mathrm{e}}\right): \dot{N}_{\mathrm{e}}+\eta N_{\mathrm{e}}+v N_{\mathrm{e}}^{2}=\dot{q}$, where $\eta$ measures removal by neutral defect trapping (analogous to attachment), and $v$ measures removal by charge capture (analogous to recombination). As a first exercise, I assumed that $\eta$ and $v$ operate significantly in separably different carrier-density regimes, so that the EM

\footnotetext{
*Note that I dimension in MKS Thus, $1 \gamma \mathrm{MeV} / \mathrm{m}^{2} \mathrm{~s} \equiv 10^{4} \mathrm{Rhu}$

${ }^{\dagger}$ We may suppress relative time because the radial dimension of significant $E_{r}$ contributions is sufficiently small $\ln$ effect, $c \rightarrow \infty$ for this piece of the problem

† ' shake" tıme-scale obtains $v=1(\mathrm{~m} / \mathrm{sh})$, conveniently

${ }^{5}(\partial \varepsilon / \partial x)$ is the gamma energy deposition density $\varepsilon_{p}$, is the classical charge-pair ionization energy
} 
field problem could be addressed in four progressive gamma-dose regimes: ambient, attachment, recombination, and "breakdown." Without further examination here, the adopted coefficient values (for the kinetic conduction state) were $\eta=2 \times 10^{10} \mathrm{~s}^{-1}$ (attachment regime) and $v=10^{-12} \mathrm{~m}^{3} / \mathrm{s}$ (recombination regime). The conduction-band state becomes especially significant in the breakdown regime; it invokes a treatment which in effect, assigns very different values to $\eta$ and $v$. The carrier lifetime in this dense test medium turns out to be $10^{-10}$ to $10^{-12} \mathrm{~s}$, depending on regime; hence the integral of the rate equation will be dominated by the removal coefficients, rather than by the generation coefficient in $f(t)$ (such as " $\alpha$ ").

Likewise, the mobility $(\mu)$ is radically different for the two carrier states. Classically, $\mu$ is expressed as $\mu=(1.5 e / m)(\sigma v)^{-1}$, where $(\sigma v)$ is the carrier collision rate.* For the kinetic-state description, I chose $\mu=10^{-4} \mathrm{~m} / \mathrm{s}$ per $\mathrm{V} / \mathrm{m}$. These definitions are sufficient so that the conductivity, transitions between regimes, and subsequent $E$-field solutions are analytically defined for the first three regimes. The fourth regime ("breakdown") yields to a self-consistent analysis; it indicates $\mu=0.1 \mathrm{~m} / \mathrm{s}$ per V/m.

The ambient regime sets $\sigma=\sigma_{\mathrm{g}}$, and the $E$-field initially rises proportionately with $\phi_{\mathrm{g}}$, as $e^{\alpha t}$. The transition to attachment occurs as $\sigma$ becomes significant: $\sigma \approx \sigma_{\mathrm{g}}+\alpha \kappa \kappa_{0}$. Well into that regime, the ratio $J_{\mathrm{c}} / \sigma$ becomes constant, defining a saturation $E$-field at $\sim 10^{7} \mathrm{~V} / \mathrm{m}$. The transition to recombination occurs when $N_{\mathrm{e}} \approx \eta / v$; within this third regime, the $E$-field would rise as $\sqrt{\phi_{\gamma}}$. However, by the choice of coefficient values, it is a narrow regime; the ionization-track density quickly satisfies a breakdown criterion, and band-conduction takes over. The conductivity then rises dramatically, such that the $E$-field drops to a much lower saturation value $\left(<10^{6} \mathrm{~V} / \mathrm{m}\right)$.

\subsection{E-Field and Source Potential}

The calculated $E_{r}(r)$ profile at peak $\phi_{\gamma}$ appears in Fig. 2-3; this instantaneous "snapshot" shows that, as this peak is approached, the several regimes develop in radial sequence in the earth medium around the test canister. Each transition gamma-flux value corresponds to a (moving) transition radius. As there is little to distinguish the $\eta$ and $v$ regimes, I merged these into a single "saturation regime." Table 2-1 shows the values at the transition points.

The transition radii are $r_{\mathrm{a}}$, corresponding to $\phi_{\gamma_{\mathrm{a}}}$, and $r_{\mathrm{b}}$, corresponding to $\phi_{\gamma_{\mathrm{b}}}$. As the source intensity increases and decreases, the regime zones move outward and back in; this is depicted by Fig. 2-4. The two transition radii can be defined analytically as functions of time by a common recursive expression:

$r_{\ell}=\lambda_{n}\left[\ln \left(\Phi_{\mathrm{b}} / \lambda_{\gamma}^{2} \phi_{\gamma \ell}\right)-2 \ln \left(r_{\ell} / \lambda_{\gamma}\right)-\ln (4 \pi)+\ln f(t)\right]$,

where $\ell=\mathrm{a}$, b; and $f(t)$ still represents the $\phi_{\gamma}$ history.

Since the description of $E_{r}(r)$ is uncomplicated within each zone, the potential integral reduces to a simple algebraic expression; with sufficient accuracy, it is as follows:

$V_{r}(t)=E_{\mathrm{s}} \lambda_{\gamma}+E_{\mathrm{s}}\left(r_{\mathrm{a}}-r_{\mathrm{b}}\right)+E_{\mathrm{b}}\left(r_{\mathrm{b}}-r_{1}\right)$

under the condition: $r_{\mathrm{a}}(t)>r_{\mathrm{b}}(t)>r_{1}$. This potential history is illustrated in Fig. 2-5 as a linear graphic. The most striking feature is the prodigious compression of the $\phi_{\gamma}$ peak. All the models, all the matter parameters, and all the calculational methods that I tested displayed this same behavior. It is intrinsic to the geometry and the electron-density of matter. The $V_{r}$ magnitude stayed within the illustration scale, even for very radical alternatives. The principal effect of different assumptions or criteria, was to shift the transition $\phi_{\gamma}$ values, without significant impact on the central zone interval $\left(r_{\mathrm{a}}-r_{\mathrm{b}}\right)$. (It has the biggest "leverage.")

This, then, is the electromagnetic driver for the Compton-driven models: the potential transient that develops between the test canister space and "the Rest of the World." The essential source quantity turns

* This " $\sigma$ " is obviously a mean atomic collision cross section. 
out to be the charge $(Q)$ that's made available between the $V_{r}(t)$ shoulders. It is well approximated by a rectangular pulse about $250 \mathrm{~ns}$ wide, furnishing about 0.05 coulombs." So (as a preview), the effective impulse current is $2 \times 10^{5} \mathrm{~A}$, as this "pulsed battery" sees an impedance of about $50 \Omega$.

\subsection{Band Conduction}

In the original development, I introduced "breakdown" because, by several independent arguments, a catastrophic change should occur in the range 5 to $20 \mathrm{MV} / \mathrm{m}$. Since then, I developed a model for such a breakdown that entails two physical concepts, as depicted in Fig. 2-6:

- An assumption of band conduction around and along the Compton ionization tracks.

- A critical $\phi_{\gamma}$ level (transition) at which the track density becomes high enough for significant overlap or "connectivity."

In that idealized model of charged-particle ionization, there is a core of dense ionization surrounded by a cylindrical tube of excitation. In an outer layer, the excitation energy would be just sufficient to raise particular electrons into a conduction band. ${ }^{\dagger}$ The band mobility is generally so high that the required carrier population is greatly reduced-by $\sim 10^{3}$.

Next, consider the (idealized) collection of ionization tracks. At some high $\phi_{\gamma}$, the average track separation becomes small enough that band-conduction continuity is established. This occurs at $\phi_{\gamma} \approx 10^{28} \gamma \mathrm{MeV} / \mathrm{m}^{2} \mathrm{~s}$. (At the entrance radius $r_{1}$, this level is attained within the alpha phase, well below the break point.) The corresponding $E$-field is $E_{v} \approx 10^{-7} \sqrt{\phi_{\gamma}}=10^{7} \mathrm{~V} / \mathrm{m}$.

Hypothetically, these two mechanisms operate to greatly increase the conductivity and (once again) lock it to the instantaneous carrier production rate. Then, $E_{\mathrm{b}} \approx J_{\mathrm{c}} / \sigma_{\mathrm{b}}=$ constant. The choice for this saturation condition is not critical, as long as $E_{\mathrm{b}} \ll E_{\mathrm{s}}$, because its contribution to the $V_{r}$ integral is then small. (Several independent considerations suggested $E_{\mathrm{b}}$ values in the range from 1 to $2 \times 10^{6} \mathrm{~V} / \mathrm{m}$. $)^{\ddagger}$

\subsection{Compton-Current Dipole Moment}

The essential gamma-flux asymmetry for the Compton-driven models is furnished by the thick superstructure of the test canister immediately above the nuclear device. As a practical example, I assumed that it shadows one steradian of gamma flux, axially symmetric about the vertical centerline, as shown in Fig. 2-7. The Compton-current dipole moment belongs to the mirror-image net current in the downward steradian of earth medium immediately beneath the test canister. To that end, I used the current density derived directly from the radial- $E$ calculation, to define a current element distribution. This source is small enough that I could treat it as entirely coherent (i.e., single point in time). The subsequent analytic development included

- Time-dependent description of the current moment and corresponding Fourier transform.

- Specification of attenuated and phase-dispersed Fourier components at the sensor locations.

- Fourier inversion (to time domain) to construct sensor signal histories.

I used the net current density obtained directly from the radial- $E$ calculations to define the currentelement distribution within that "downward steradian." The effective dipole strength was then obtained as the following sum:

$p=\Sigma i \delta \ell=\sum_{\infty}^{R}\left(J_{\text {net }} / r^{2}\right) d r$.

\footnotetext{
*A reasonable case can be made for much later "tail" contributions by "slow" currents traceable to the decay of fission products and neutron induced radioactivity But that's another story

${ }^{\dagger}$ The existence of such a band (or bands) and the lifetıme of carriers therein are separate questions not addressed here

tOther mechanısms can be invoked for a 'breakdown" response at $\sim 10^{7} \mathrm{~V} / \mathrm{m}$, such as sparkıng along the electronically "dırty" surfaces of the $\mathrm{SiO}_{2}$ microcrystals that comprise the bulk of the alluvium
} 
However, $J_{\text {net }}=J_{\mathrm{c}}+\sigma E=(-) \kappa \kappa_{0}(\partial E / \partial t)$, so most of the current dipole contribution comes from the outermost unsaturated zone, where $(\partial E / \partial t)$ is still significant.* By calculational experience, $|\sigma E| \ll \mid J_{d}$ up to the last $\phi_{\gamma}$ $e$-fold before saturation; within that one generation, $\partial E / \partial t$ collapses to nearly zero. Hence, I set $J_{\text {net }} \equiv J_{c}$ and truncated the sum at the first transition radius, as $R=r_{\mathrm{a}} . J_{\text {net }}$ then reduces to an obvious analytic form: $J_{\text {net }}=\left(j \phi_{\mathrm{a}}\right)\left(x_{\mathrm{a}} / x\right)^{2} e^{-\left(1 \mathrm{l}_{\mathrm{a}}\right)}$, where $x=r / \lambda_{\gamma}$. Thus, for this EM source, the principal role of the $E$-field model is to describe the history of the outer transition radius, appearing here as $x_{\mathrm{a}}$.

The dipole-moment expression reduces at once to

$p=p_{\mathrm{a}} x_{\mathrm{a}}^{2} \int_{0}^{\infty} e^{-u} d u=p_{\mathrm{a}} x_{\mathrm{a}}^{2}(t)$

where $x_{\mathrm{a}}=r_{\mathrm{a}} / \lambda_{\gamma^{\prime}} p_{\mathrm{a}}=\rho \lambda_{\gamma}^{3} \phi_{\gamma}$. For our fiducial case, $p_{\mathrm{a}} \approx 85 \mathrm{~A} \cdot \mathrm{m}$. This is not a normalized expression; the maximum $r_{\mathrm{a}}$ penetration obtains $x_{\mathrm{a}} \approx 12$, whence $p_{\max } \approx 1.2 \times 10^{4} \mathrm{~A} \cdot \mathrm{m}$. Dipole histories are depicted in Fig. 2-8 for several $\sigma_{g}$ values; on the semilog scale, the best-fit analytic function is the sawtooth bounded by $t=0$ and the decaying exponential: $p=p^{*} e^{-a t}$, where $a=64000 \mathrm{~s}^{-1}$. ( $p^{*}$ is an empirically adjusted slope-intercept. $)^{\dagger}$

\subsection{Sensor Signals}

In setting up the calculation of the representative sensor signals for both Compton-driven models, I developed a convenient Fourier formalism to properly account for the strong dispersion of these sharply transient current-dipole fields. In both cases, the analytic representation of the dipole source is the exponential decay (as just above), for which the Fourier transform is

$F T_{\omega}=\frac{a-i \omega}{a^{2}+\omega^{2}}=|F| e_{\omega}^{i \phi}$

where $|F|=\left(a^{2}+\omega^{2}\right)^{-1 / 2}$, and $\phi_{\omega}=\arctan (\omega / a)$.

This is "propagated" to the sensors with appropriate geometric and attenuation factors; in collective form, the inverse Fourier transform is

$E(t), H(t)=\frac{\left[e_{0}, h_{0}\right]}{2 \pi} \int_{-\infty}^{\infty}\left\{\mathrm{FT}_{\omega} G_{\omega} \mathrm{AT}\right]_{\omega} e^{t \omega t} d \omega \rightarrow\left[E_{0}, H_{0}\right] S(t)$

where

$G_{\omega}$ is the source-observer geometric factor,

$\mathrm{AT}_{\omega}$ is the complex Von Hippel $(\mathrm{VH})$ attenuation,

$e_{0}, h_{0}$ are the dipole field coefficients $\left(e_{0}=p_{0} / 4 \pi \sigma_{g} d^{3}, h_{0}=p_{0} / 4 \pi d^{2}\right)$,

$E_{0}$ and $H_{0}$ collect a rabble of coefficients $\left[E_{0}=0.0020 \mathrm{~V} / \mathrm{m}, H_{0}=0.00070 \mathrm{At} / \mathrm{m}\right.$ (for $\left.\left.\sigma_{\mathrm{g}}=0.0025\right)\right]$.

The real and imaginary components can be factored into the following general form:

$\mathrm{IFT}=\frac{1}{2 \pi} \int_{0}^{\infty} \operatorname{Arg}_{(\nu} e^{i \phi_{t \prime \prime} e^{\prime(\omega t}} d \omega$

The real waveform becomes

$S(t)=\frac{1}{\pi} \int_{0}^{\infty} \operatorname{Arg}_{\omega} \cos \left(\omega t+\phi_{\omega}\right) d \omega$

\footnotetext{
*This essentral point also shows why the Compton current emitted from the canister wall is "invisible."

"This approximation suppresses the fast intial nse; by test, its Fourier sıgnal components vanish by much greater attenuation relative to the slower " $\mathrm{a}$ " components
} 
The normalization addresses the (real) coefficient $\operatorname{Arg}_{\omega}$ so that it converges to unity in the quasi-static limit.

The phase term $\left(\phi_{\omega}\right)$ is very frequency-sensitive; the Nyquist phase trace spins around rapidly as the frequency increases (in the incremented integration). This made the computations extremely sensitive to the precision of frequency incrementation. To eliminate the associated IFT scatter, I resorted to phase incrementation (as $\phi_{n+1}=\phi_{n}+\delta \phi$ ). The frequency variable $(\omega)$ is then defined (in non-regular increments) by inverting the $\phi_{\omega}$ (arctan) expression. The subsequent eigenfunction arithmetic is extensive and somewhat complicated." I used the "Flat Fourier," $\mathrm{FT}_{\omega}=1$, as the basic test problem, along with a normal Von Hippel function: $e^{-(1+j) r d}$. It defines the characteristic frequency: $\omega_{\mathrm{c}}=2 / \mu_{0} \sigma_{\mathrm{g}} d^{2}$. The phase initialization was set so that all $\omega$ phases converged to zero at the source for $t=0$.

This IFT arithmetic for the Compton dipole signals is considerably simpler (than for the Casingcurrent model, next) because all signal components originate from the same "downhole- $/$ ero" (DHZ) location. As finally expressed, the Local sensor signals for the fiducial case are $E_{\rho}(t)=E_{0} S_{\mathrm{v}}(t)$, $H_{\phi}(t)=H_{0} S_{\mathrm{h}}(t)$, and $B(t)=\mu_{0} H_{0}\left(\Delta S_{\mathrm{h}} / \Delta t\right) . S_{\mathrm{e}}$ and $S_{\mathrm{h}}$ are the IFT integrals, further expressed as

$S_{\mathrm{e}}=\sum_{m}\left\{\frac{1+\sqrt{2 x}}{e^{\vee 2 x}} \sqrt{\frac{1+2 x^{2}}{1+x^{4} / 16}}\right\}_{m} \Delta_{\mathrm{m}}^{\mathrm{e}} \cos \phi_{\mathrm{m}}^{\mathrm{e}}$,

and

$S_{\mathrm{h}}=\sum_{m}\left\{\frac{1}{e^{\sqrt{2 x}}} \sqrt{\frac{1+2 \sqrt{2} x+4 x^{2}}{1+x^{4} / 16}}\right\}_{m} \Delta_{\mathrm{m}}^{\mathrm{h}} \cos \phi_{\mathrm{m}}^{\mathrm{h}}$,

in which $x_{n t}^{2}=\omega_{m} / \omega_{c}, \Delta_{m}=x_{m+1 / 2}^{2}-x_{m-1 / 2}^{2}$.

The Eigenfunctions are summarily defined in Appendix A. $S_{\mathrm{e}}$ and $S_{\mathrm{h}}$ are not intrinsically normalized, so that $E_{0}$ and $H_{0}$ do not indicate the true peak amplitudes. Figures 2-9 and 2-10 illustrate these signal histories for various cases; peak values are indicated there and in the summary (Table 2-2). I show one polarity reversal in detail, to indicate its generally small size. ${ }^{+}$The magnetic amplitude $(H)$ is insensitive to $\sigma_{\mathrm{g}}$ (although $\dot{B}$ behaves rationally, along with $E$ ). $\bar{\omega}$ measures the principal frequency of the initial excursion, as $\bar{\omega}=(\dot{S} / S)_{\text {peak }}$.

In principle, these Local Compton-dipole signals can be classed as "visible"; however, in practice, they would be overlain by the casing-current fields, which turn out to be about two orders-of-magnitude larger $(\times 100)$. The amplitude coefficients for the Distant sensor signals have these tiny (invisible) values: $E_{0}=0.8 \times 10^{-9} \mathrm{~V} / \mathrm{m}, H_{0}=0.3 \times 10^{-9} \mathrm{At} / \mathrm{m}$. I did not calculate their waveforms.

\footnotetext{
${ }^{*}$ I note the results below (and in Section 3) as the signal time functions $S_{\mathrm{e}}(t)$ and $S_{\mathrm{h}}(t)$; these are further defined in brief appendices to the two Compton sections.

'Because of the excellent convergence of the IFTs over the entire $\omega$ range, I consider this reversal as "real"-not a calculational anomaly.
} 
Table 2-1. Transition values.

\begin{tabular}{llccc|} 
Regime transition & $\phi_{\gamma}\left(\gamma \mathrm{MeV} / \mathrm{m}^{2} \mathrm{~s}\right)$ & $J_{\mathrm{c}}\left(\mathrm{A} / \mathrm{m}^{2}\right)$ & $\sigma(\mathrm{mho} / \mathrm{m})$ \\
\hline Ambient-saturation & $\phi_{\gamma_{\mathrm{a}}}=1.4 \times 10^{25}$ & $1.4 \times 10^{4}$ & 0.003 \\
Saturation-breakdown & $\phi_{\gamma_{\mathrm{b}}}=$ & $10^{28}$ & $10^{7}$ & $\sim 1.0$ \\
\hline
\end{tabular}

Table 2-2. Compton-dipole Local-signal amplitudes.

\begin{tabular}{|cccccc|}
\hline$\sigma_{\mathrm{g}}$ & $\omega_{\mathrm{c}}$ & $\begin{array}{c}E_{\max } \\
(\mathrm{V} / \mathrm{m})\end{array}$ & $\begin{array}{c}H_{\max } \\
(\mathrm{At} / \mathrm{m})\end{array}$ & $\begin{array}{c}\dot{\beta}_{\max } \\
\left(\mathrm{Wb} / \mathrm{m}^{2} \mathrm{~s}\right)\end{array}$ & $\bar{\omega}$ \\
\hline 0.0025 & 16000 & $9 \times 10^{-3}$ & $1.5 \times 10^{-3}$ & $10^{-4}$ & $1.1 \times 10^{5}$ \\
0.005 & 8000 & $5 \times 10^{-3}$ & $1.5 \times 10^{-3}$ & & \\
0.01 & 4000 & $2.5 \times 10^{-3}$ & $1.5 \times 10^{-3}$ & $4 \times 10^{-5}$ & $4.4 \times 10^{4}$ \\
\hline
\end{tabular}




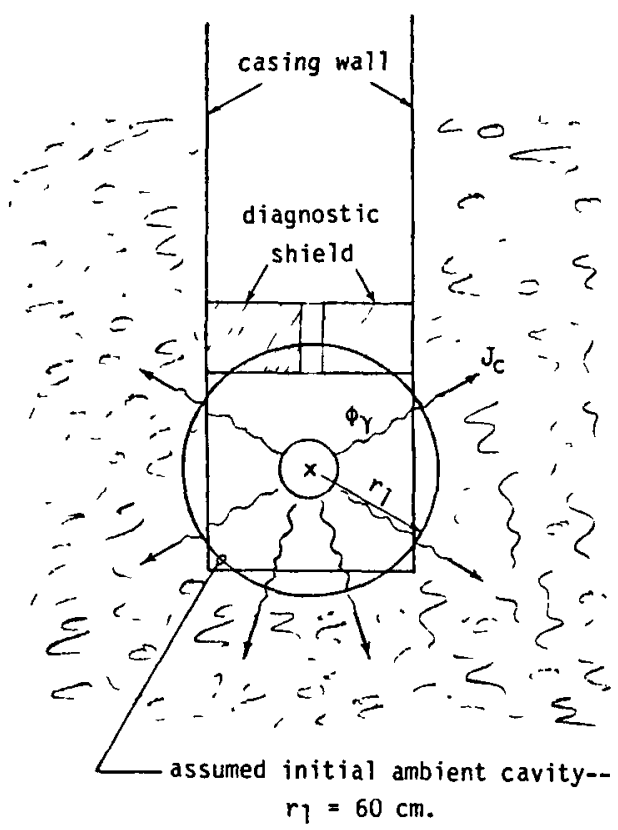

Figure 2-1. A spherical canister surrogate.

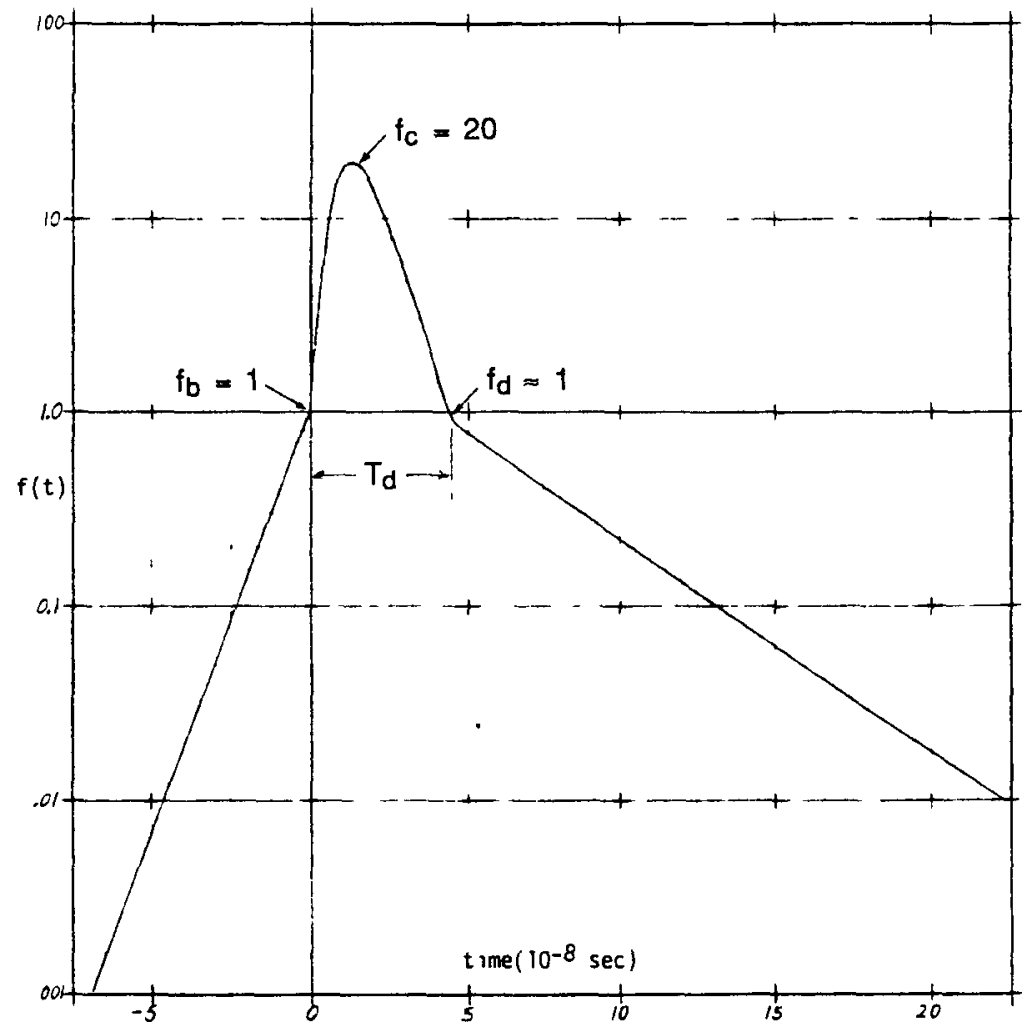

Figure 2-2. Assumed gamma source history: Normalized $\phi_{\gamma}$ as $f(t)$. 


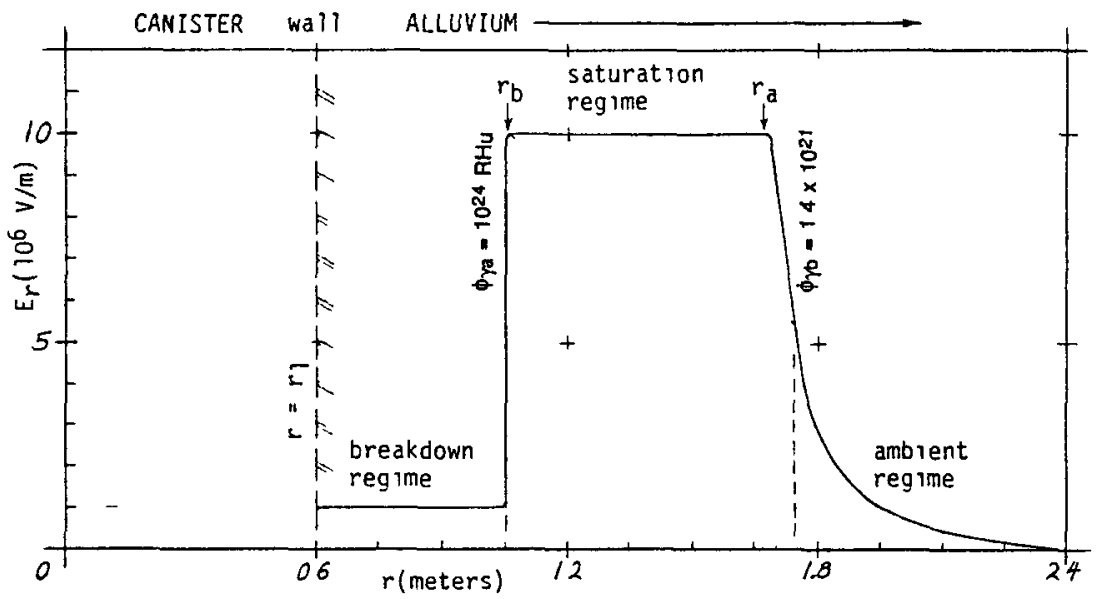

Figure 2-3. Calculated $E_{r}(r)$ at gamma peak.

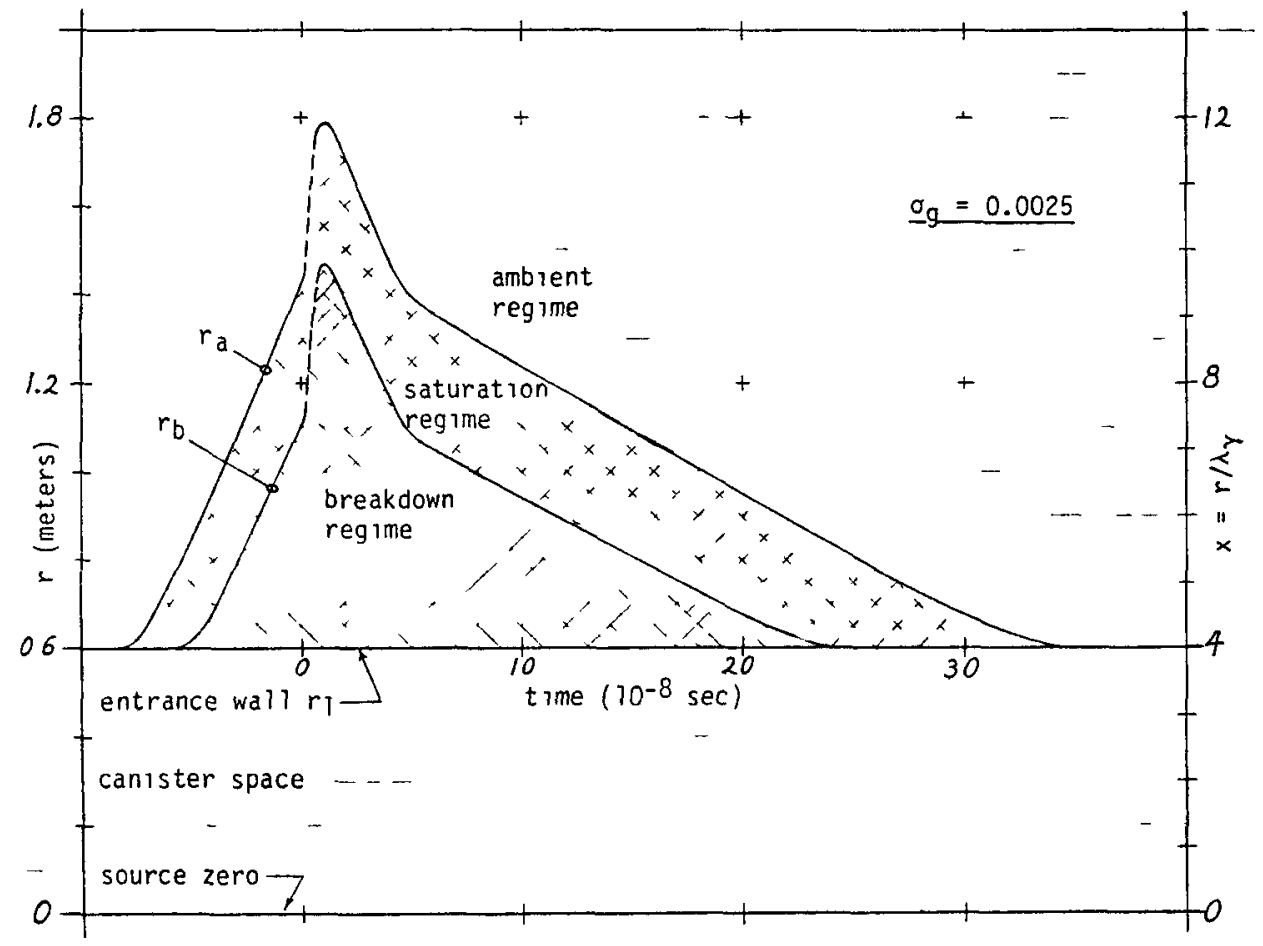

Figure 2-4. Typical transition-radius history. 


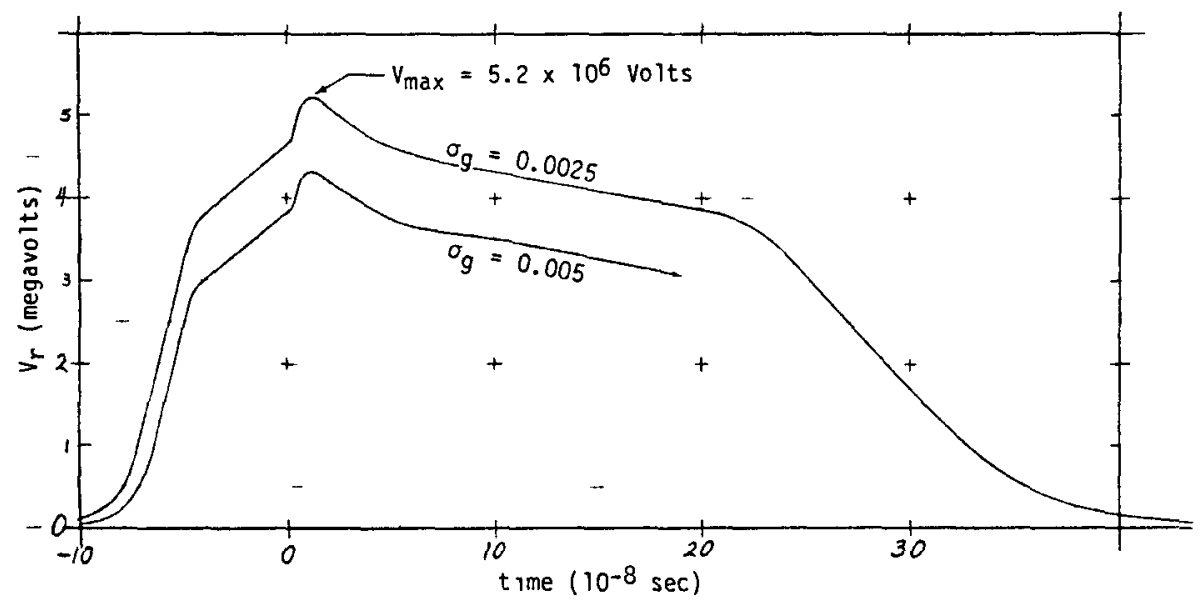

Figure 2-5. Typical source potential function $V_{r}(t)$.

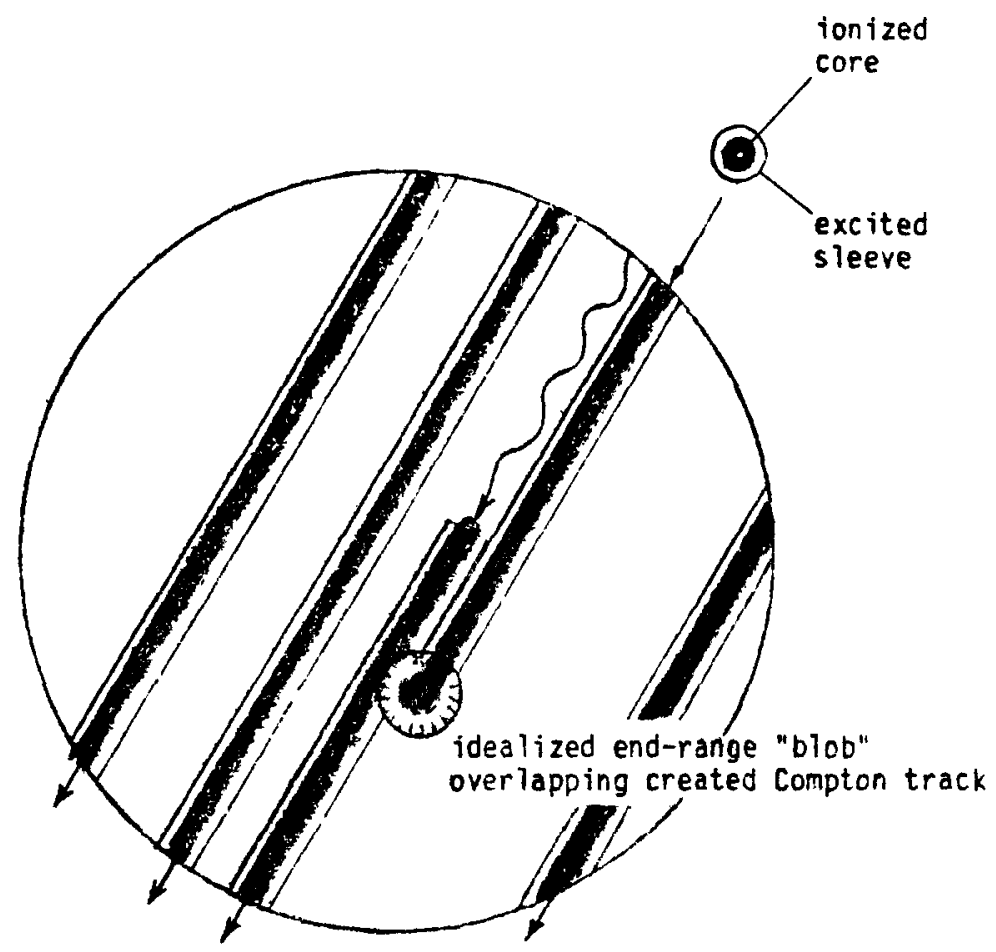

Figure 2-6. Idealized Compton-ionization tracks. 


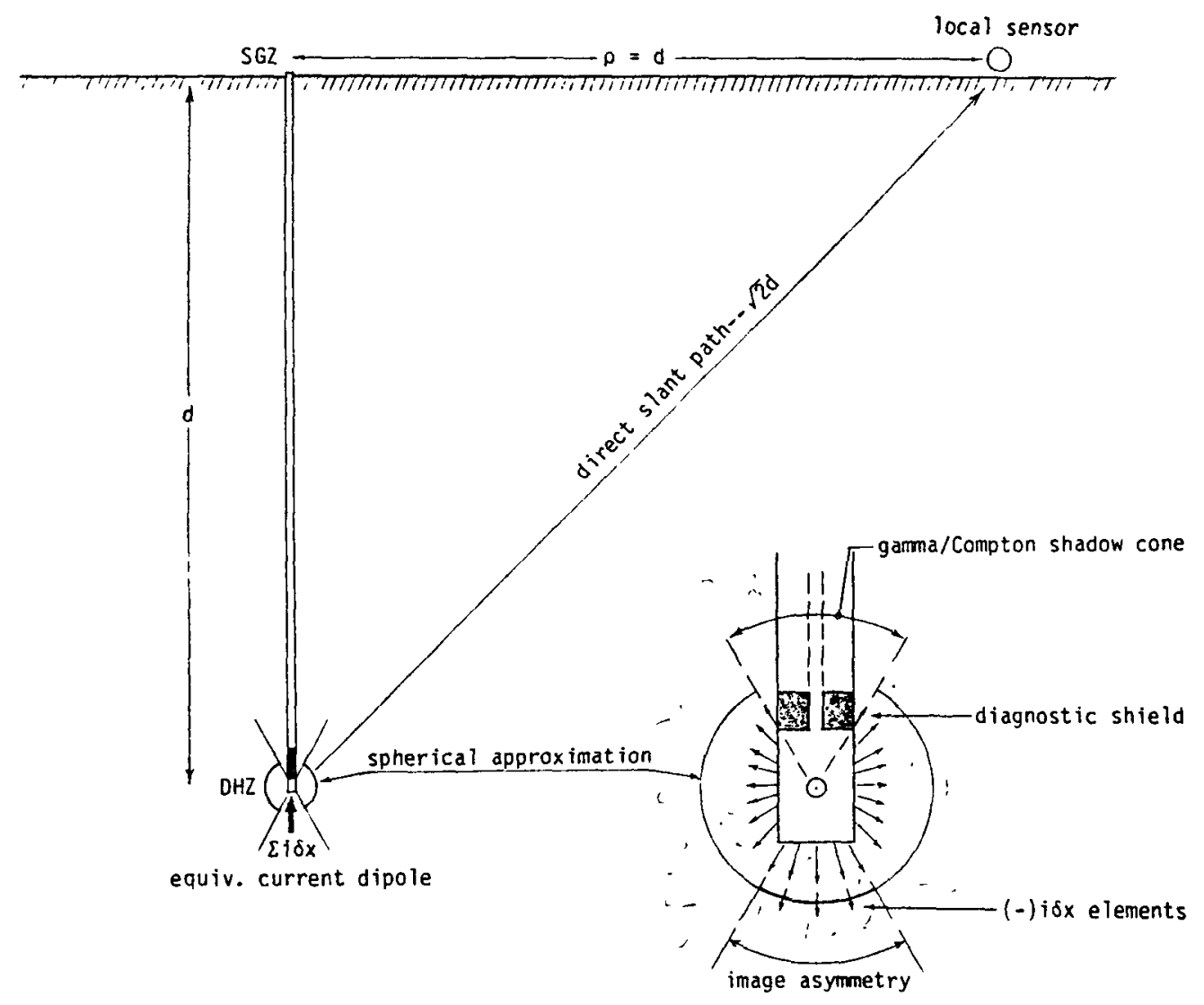

Figure 2-7. The Compton-current dipole. 


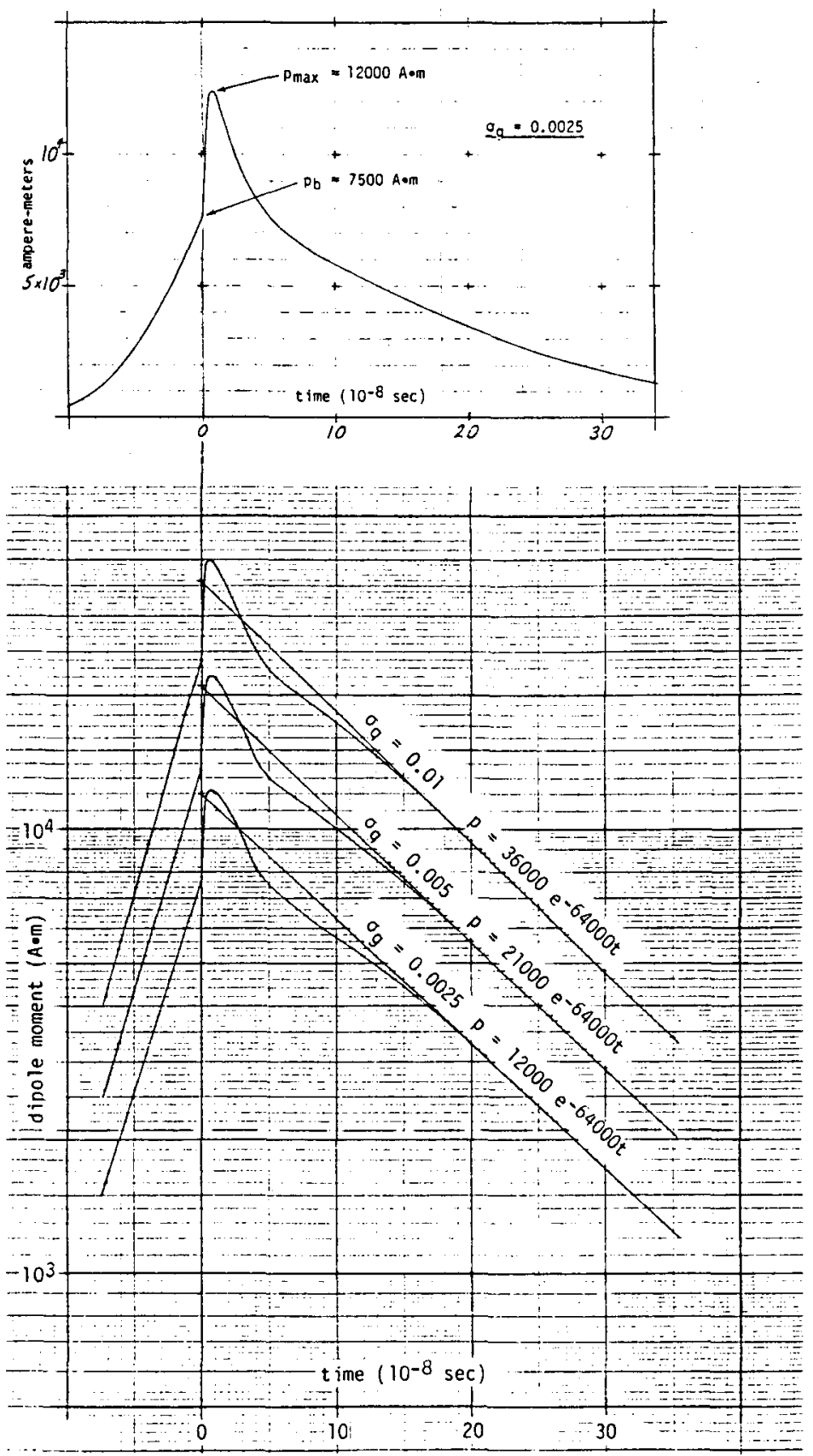

Figure 2-8. Compton dipole-moment histories. 

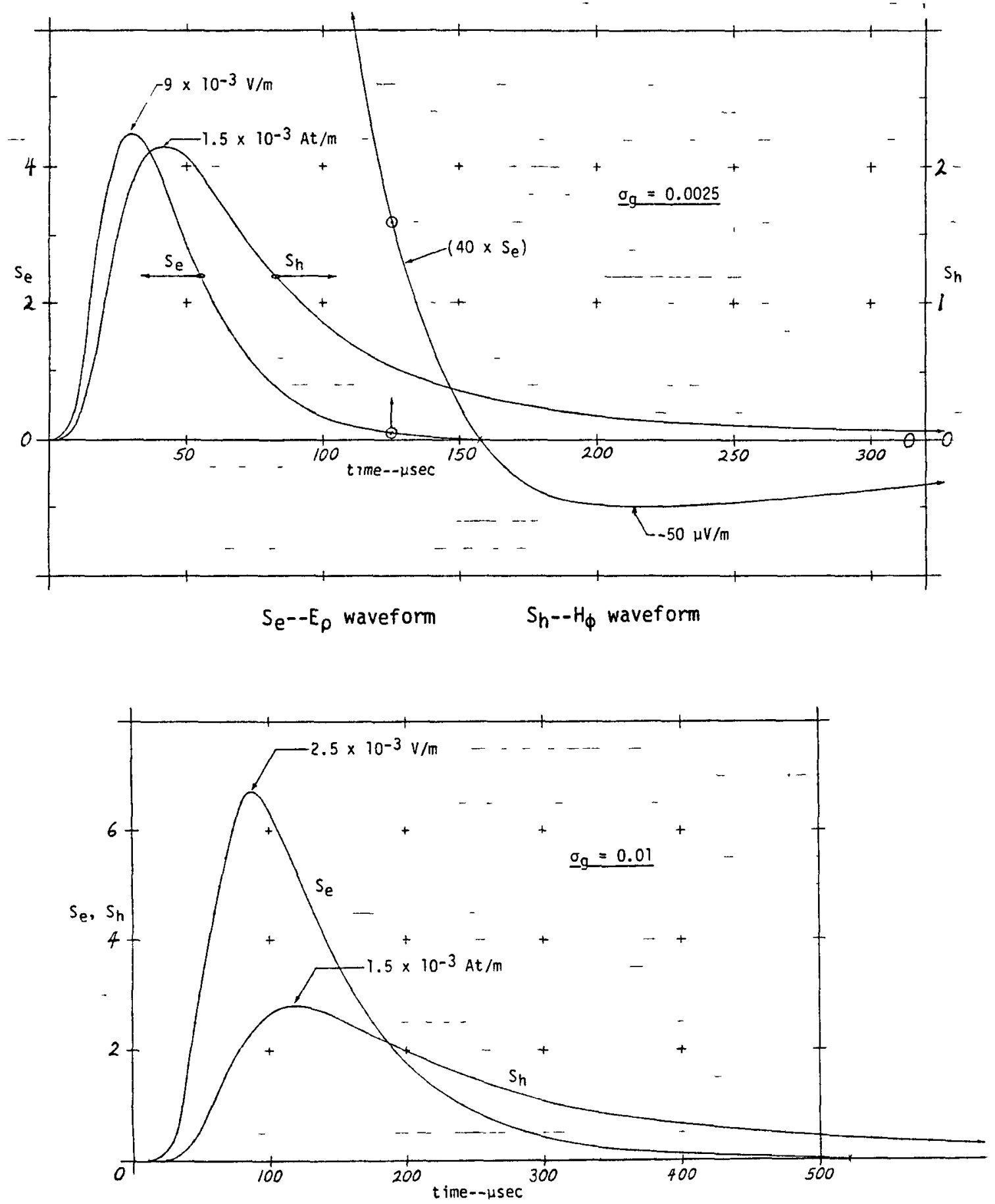

Figure 2-9. Local signal waveforms (IFT functions)-Compton dipole. 


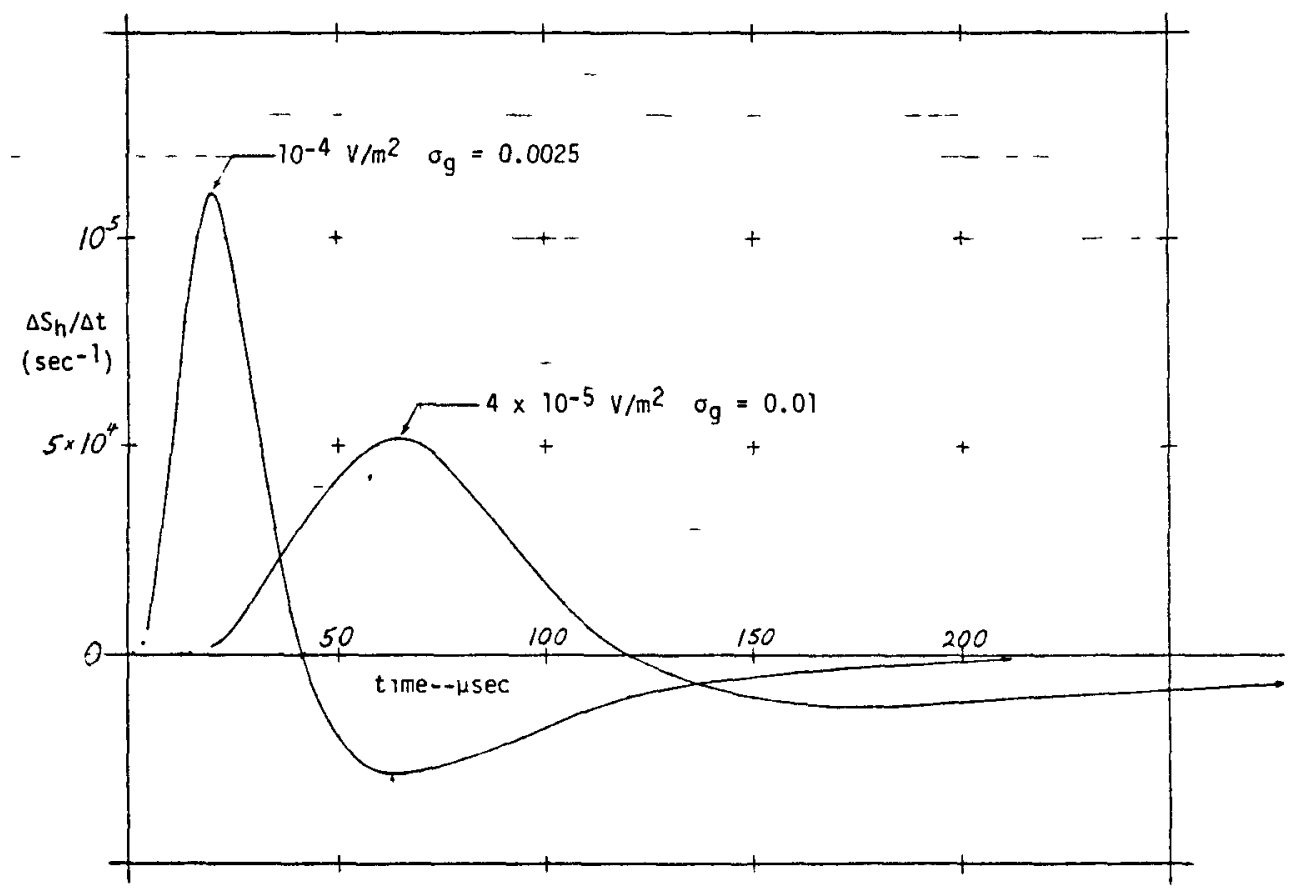

Figure 2-10. Local B́ waveforms-Compton dipole. 


\section{The Casing-Current Fields}

The calculation of the casing-current signals was considerably more complicated (as compared to the Compton dipole). At a given instant, a Local sensor sees EM field contributions from current elements distributed along the entire length of the vertical conductors in the test drill hole, as depicted in Fig. 3-1. The relative times of origin of those current elements and the relative propagation times to the sensor are significant here. Contributions along the "up-and-over" path must also be evaluated. These analyses included

- Time-dependent description of the currents injected by the DHZ charge impulse and propagated upward.

- Fourier description of the field contributions at a sensor from the vertical array of equivalent current elements.

- Fourier inversion of the sums of the field components to the time domain.

Again, these calculations were most sensitive to the tracking of the phase of the Fourier components. While I found it possible (and reasonable) to adopt various amplitude approximations, phase had to be meticulously defined. To that end, I again incremented the computations in the phase domain, using the eigenfunction formalism to define Fourier frequencies and their intervals, as dependent variables.

I also carried out some independent calculations of Local sensor signals from the casing-current distribution using a diffusion approximation, entirely in the time domain. This technique has certain limitations that make it principally useful as a verification of the Fourier scheme.

Here I interject a clarification. Uncased test emplacements behave electromagnetically like cased ones. In our fiducial case, a uniform, continuous, cylindrical, drill-hole casing constitutes the outermost conductor. In an uncased system, the uphole conductor includes the close-coupled combination of the suspension drill-pipe and the thick control/diagnostic cable bundle. The electromagnetic impedance $\left(Z_{c}\right)$ depends on the logarthm of the effective radial dimensions. Hence, $Z_{c}$ is a soft function, and it is not much different for the two situations.

\subsection{The Heaviside-Sundé Solutions}

The analysis of the propagation of current impulses (or "surges") along immersed conductors was originally carried out by Heaviside (circa 1870), as that concerned the performance of single-conductor trans-oceanic telegraph cables. It was further refined by Carson (1926) and definitively summarized by Sundé (1949), all at Bell Telephone Laboratories. ${ }^{* 5}$ Heaviside's ignorance of displacement is inconsequential, as long as $\sigma_{\mathrm{g}} \gg \kappa \kappa_{0} \omega$ within the transmissible frequency domain. That translates to a validity criterion for the description of the current distribution along a conductor: $x>\left(\mathrm{Z}_{0} \sigma_{\mathrm{g}} / \sqrt{\kappa}\right)^{-1} \approx 1.7 \mathrm{~m}$. The invalid region is thus buried within the source $E$-field zone.

Sundé defines two functions that are directly applicable:

- The surge impedance presented by a buried conductor to a step-function current generator;

- The current history along a buried conductor due to a unit step-function input current.

Figure 3-2 depicts the casing as a low-impedance conductor penetrating into the $E$, zone. I calculated the injected surface-current impulse by analogy to a long cable connected to a transient-voltage generator (or "pulsed battery"):

$I_{c}=\frac{V_{r}(t)}{R(t)+Z_{c}\left(t^{*}\right)}$

where $R(t)$ is the battery resistance between source and infinity, $Z_{c}\left(t^{*}\right)$ is the Sundé impedance presented by the long cable, $t^{*}$ indicates that $Z_{c}$ is reactive-it depends on past history so that $I_{c}$ must be calculated as a convolution-and $V_{r}(t)$ is the potential integral of $E_{r}$, as expressed in Section 2.

\footnotetext{
* This approach is analytically sımpler in companson to a complete, tıme-dependent, boundary-value exercise "starting from scratch", it jumps ahead by presuming that we know what the important parts of the answers will be, and assigning well-tested solutions to them
} 
I approximated the source resistance as that between a conducting sphere and infinity; that is a well-known elementary problem which obtains $R(t)=\left(4 \pi \sigma r_{a}\right)^{-1}$. I assigned $r_{\mathrm{a}}$-the saturation-transition radius-to this representation because contributions from smaller shells $\left(r<r_{a}\right)$ belong to much higher conductivities. Initially, the ambient source resistance would be $R \approx 50 \Omega$ (for $\sigma_{g}=0.0025$ ).

I transliterated Sundé's impedance expression as follows [see Ref. 5, Eq. (8.18), p. 264]:

$Z_{c}\left(t^{*}\right)=(\pi \sqrt{2 \pi} \sigma a)^{-1} \sqrt{s} \ln (1.12 / s)$

where $s=\beta T=\mu_{0} a^{2} \beta / 16, a$ is the casing radius, and $\beta$ is a rate-of-change, which has the consequence that each potential increment sees an impedance that depends on its instantaneous value. Calculated impedance values for typical $\beta$ ranges are 10 to $20 \Omega$.*

The input-current convolution was computed using

$I\left(t_{n}\right)=\sum_{m} \frac{\Delta V_{n-m}}{R_{n}+Z_{m}}$

As an auxiliary input for $Z_{m}, \beta_{m}$ was approximated as

$\beta_{m}=\frac{\Delta V_{n-m}}{\Delta V_{n-m} \Delta t}$

The behaviors of $Z_{\mathrm{c}}$ and $R$ through the gamma-pulse phases for our fiducial case are illustrated in Fig. 3-3; Fig. 3-4 shows the results of some input-current calculations. These figures illustrate how severely the peak phase is truncated in $I_{c}(t)$; it is now a mere pimple. The subsequent current diffusion up along the electromagnetic outer "skin" of the casing is so severely dispersive that even this structure is quickly lost. The current envelope beyond $\sim 20 \mathrm{~m}$ becomes proportional to the input time-integral-that is, to the injected charge; it is equally well expressed as an equivalent rectangular impulse:

$Q=\Sigma_{n} I\left(t_{n}\right) \Delta t=I_{0} T$

Here, $T$ is the width across the $I_{c}(t)$ "shoulder."

\subsection{Casing-Current Distribution}

Next in Sundé's notation, the current history at a point $x$ along a conductor-for a unit step-function input-is expressed as [see Ref. 5, Eq. (8.08), p. 259]

$$
\begin{aligned}
& P(x, t)=e^{-\alpha x}+\alpha x \int_{x}^{v t} \frac{e^{-\alpha u}}{W} I_{1}(\alpha W) d u \\
& \alpha=\frac{\sigma_{\mathrm{g}}}{2 \sqrt{\kappa}} Z_{0}, \quad W=\sqrt{u^{2}-x^{2}}
\end{aligned}
$$

where $x$ is the actual distance along the casing (in meters); $x=0$ at the current source; $\kappa=9$; and $Z_{0}=377 \Omega$. $I_{1}$ is the modified complex Bessel function of the first kind and first order; $u$ is a running one-dimensional line-variable of integration between the farthest propagation-time point $v t$ and the independent line

\footnotetext{
* The penetration of the $E_{r}$ zone by the casing may appear to "short-circuit the battery" to some degree around the edges-note Fig 3-2 A rough estimate of that shunting resistance is $\left(2 \pi a \sigma_{h}\right)^{\prime} \approx 100 \Omega$, such that the leakage current should be much less than the injected casing current furthermore, the penetration lies at the edge of the collimator shadow cone (Fig 2 -1) so that it is not as strongly affected by the gamma illumination Hence, I disregarded this second-order feature
} 
coordinate $x$ (n.b., this $\alpha$ is neither the exponential $\alpha$ nor the diffusion $\alpha$ ). In effect, the integral translates and disperses to the point $x$, all of the delayed current contributions that reach $x$ at time $t$ from all timeeligible line elements.

I describe the charge impulse into the casing conductor as a pair of equal and opposite step functions, of amplitude $I_{0}$, delayed by the rectangular impulse time $T$. Then, at line coordinate $x$, we see

$\left.I_{0} \Delta P_{1}(t)=I_{0} \mid P_{+}(x, t+T)-P_{-}(x, t)\right]$.

A sufficiently short impulse thus examines the waveform at $x$ as the derivative of the Sunde function.

Some mathematical manipulations lead to a simplified expression for the impulse residue. ${ }^{* 6} I$ also change the time frame to units of $10^{-8}$ s ("shakes"), so that $v=c / \sqrt{\kappa}=1 \mathrm{~m} / \mathrm{sh}$. That obtains

$i(x, t)=I_{0} \Delta P_{x}=0.40\left(\alpha^{2} x v\right) Q\left[\frac{1-0.375 \alpha W}{\alpha W \sqrt{ } \alpha W} e^{\alpha(W-t)}\right]$.

It is a straightforward computational exercise; Fig. 3-5 depicts a typical sequence of calculated current waveforms along the casing conductor, for a nominal injected charge of $Q=I_{0} T=0.05$ coulombs. Note the drift in peak time, as well as the attenuation/dispersion as the pulse makes its way up. These profiles can be analytically fitted by functions of the general class $\left(e^{-a t}-e^{-b t}\right)(b>a)$. Various of these impulse features are plotted as functions of casing coordinate in Fig. 3-6. The top of the casing ( $x=200 \mathrm{~m}$ in this fiducial example) represents a reflection discontinuity; almost as much current is reflected back down as is incident at SGZ.

\subsection{A Fictitious Casing-Current Dipole Moment}

Going on to the sensor signals, a meticulous calculation would Fourier-transform the current histories at an incremented sequence of casing points, and express the field contributions as those from corresponding dipole elements. They would be attenuated and dispersed to the sensor locations, and their sums would be inverse-transformed to obtain the "observed" pulse time structure. (All the while keeping meticulous track of phases!) Such an analysis obviously does not permit the specification of a single equivalent dipole moment.

As a matter of practicality, I sought an alternative approach that preserved the significant electromagnetic features with acceptable fidelity, yet offered a much less complicated arithmetic. The pragmatic justification for the adopted artifice is that it generates signal pulses similar in amplitude and waveform to those calculated by the diffusion approximation. (The latter formalism invokes assumptions of a different class.) The essence of the scheme is that (with appropriate phase constraints) the FT of the sums can be substituted for the sum of the FTs.

By analogy to the Compton dipole, I first calculated a fictitious current-dipole moment as the sum of the string of current elements $i_{m} \delta x$ located along the vertical conductor (per Fig. 3-1), expressed as $p(t)=\Sigma i_{m} \delta x=I_{0} L$, where $L$ is an equivalent dipole length. It is an instantaneous "snapshot," which assumes that the casing is short compared with the principal visible wavelengths $(d \gg v T)$. That also places $p$ near the center point $(d / 2)$. Figure 3-7 illustrates the results of this calculation for three $\sigma_{\mathrm{g}}$ values. Table 3-1 summarizes the coefficients for the principal slope component, now expressed as $p=I_{0} L_{0} e^{-a t}$, where $L_{0}$ is the $y$ intercept. This fictitious dipole moment is very strong: $p_{0}=I_{0} L_{0} \approx 2 \times 10^{5} \mathrm{~A} \cdot \mathrm{m}$.

Inspection of Fig. 3-5 shows at once that the dimensional criterion is violated along the lower part of the casing. Furthermore, the relative path times are completely ignored by the above approximation. The Fourier representation suggested a way to account for all that. But first, the Fourier transform for $p(t)$ remains functionally the same as for the individual dipole elements (and the Compton dipole):

\footnotetext{
* This include's factoring out the argument of the Sundé integrals so that $\int d u \rightarrow v T$, and introducing a Bessel expansion. ${ }^{6}$ There is also a validity criterion, easily satisfied over the time-space scale of our fiducial case. $\alpha W>3.75$ or $v t>x \sqrt{1}+(375 / \alpha x)^{2}$.

${ }^{\dagger}$ As in the Compton dipole, 1 suppressed the early rise Also, note that the slope ratio is invariant: $a / \omega_{\mathrm{c}}=5$ The reason is that $a$ tests the medium in the same way as $\omega_{\mathrm{c}}$, the EM energy resides in the same earth for both modes
} 
$\mathrm{FT}_{\omega}=\frac{a-i \omega}{a^{2}+\omega^{2}}$

The "small dipole" may now be located at different places along the casing, for different frequencies. As the Fourier transform entrains higher frequencies, I can assign their origins to successively lower "center points" along the casing. To do this, I introduce a normalized geometric "path factor" $R$, which measures the effective source depth for each Fourier frequency:

$R=1-\left(4+\omega / \omega_{c}\right)^{-1 / 2}$

where $\omega_{\mathrm{c}}=2 / \mu_{0} \sigma_{\mathrm{g}} d^{2}$. (The actual depth would be $R d=d-x_{\omega}$.) $R$ converges to $1 / 2$ (halfway down) for the lowest $\omega$, converges to 1 (all the way down) for the highest $\omega$, and displays a VH behavior in between.

\subsection{Sensor Signals}

In contrast to the Compton dipole (where everything always stays in the same place), I found it essential to split the path factors as to amplitude and phase. The basic reason for this is that in the signal considerations, the amplitude is attenuated only from an effective (and changing) dipole location to the sensor, whereas the phase must be counted from the origin at DHZ. It was the only specification that achieved excellent convergence in the Inverse Fourier Transforms.* For a Local sensor, the overall pathphase shift from DHZ is then defined by the normalized geometric phase factor $\boldsymbol{R}_{\phi}{ }^{\dagger}$ :

$R_{\phi}=(1-R)+\sqrt{1+R^{2}}$,

being the sum of the vertical and slant segments. For the Distant case, the paths converge to an up-andover definition, such that $R_{\phi} \equiv 1$.

The signal analysis proceeded from the same basic IFT prescription as defined earlier for the Compton dipole. I express the sensor fields for the fiducial case analogously:

$E(t)=E_{0} S_{\mathrm{e}}(t), \quad H(t)=H_{0} S_{\mathrm{h}}(t), \quad \dot{B}(t)=\mu_{0} H_{0}\left(\Delta S_{\mathrm{h}} / \Delta t\right)$.

$E_{0}$ and $H_{0}$ are amplitude coefficients for Local and Distant sensors, as follows:

Local: $\quad E_{0}=(1 / \pi) e_{0} G_{\mathrm{e}}\left(\omega_{\mathrm{c}} / a\right)=0.052 \mathrm{~V} / \mathrm{m}, \quad H_{0}=(1 / \pi) h_{0} G_{\mathrm{h}}\left(\omega_{\mathrm{c}} / a\right)=0.020 \mathrm{At} / \mathrm{m}$.

Distant: $\quad E_{0}=\left(\frac{2 \sqrt{ } 2}{\pi}\right) e_{0}\left(\frac{d}{p}\right)^{2}\left(\frac{\omega_{\mathrm{c}}^{2}}{\omega_{\mathrm{g}} a}\right)=0.016 \mu \mathrm{V} / \mathrm{m}, \quad H_{0}=\left(\frac{2}{\pi}\right) h_{0}\left(\frac{d}{p}\right)^{2}\left(\frac{\omega_{\mathrm{c}}^{2}}{\omega_{\mathrm{g}} a}\right)=6 \times 10^{-9} \mathrm{At} / \mathrm{m}$.

$\omega_{\mathrm{g}}=\sigma_{\mathrm{g}} / \kappa_{0}$ (for the air path), and $e_{0}$ and $h_{0}$ are the fictitious dipole amplitudes: $e_{0}=p_{0} / 4 \pi \sigma_{\mathrm{g}} d^{3}=1 \mathrm{~V} / \mathrm{m}$, and $h_{0}=p_{0} / 4 \pi d^{2}=0.5 \mathrm{At} / \mathrm{m} . G_{\mathrm{e}}$ and $G_{\mathrm{h}}$ are geometric terms (Local case only): $G_{\mathrm{e}}=\left(3 / R^{4} \sqrt{ }\left(\boldsymbol{R}^{2}-1\right) / R^{2}=\right.$ 0.82 and $G_{h}=1 / R^{3}=0.61 . R$ is the amplitude path factor. For the Local case, it follows the slant path: $R=\sqrt{1+R^{2}} \ddagger$ For the Distant case, it follows the "up-and-over" path; for which $R \equiv R . S_{\mathrm{e}}$ and $S_{\mathrm{h}}$ are the IFT integrals, expressed in consolidated form as an incremental sum:

$S_{\mathrm{eh}}=\sum_{m}\left[\frac{f(x)_{\mathrm{eh}}}{e^{R x} \sqrt{1+x^{4} / 25}}\right]_{m} \Delta_{m} \cos \theta_{m}$

Again, $x_{m}^{2}=\omega_{m} / \omega_{c}, \Delta_{m}=x_{m+1 / 2}^{2}-x_{m-1 / 2}^{2}$.

* This recovers the definition of Fourier phase, which was lost by the artificial collective treatment of the dipole elements for each frequency (The zero phase initialization at $t=0$ and $x-0$ (DHZ) was explicitly written into the IFTs)

${ }^{\dagger}$ Notation path factors are indicated by bold $\boldsymbol{R}$

$\ddagger$ Over the central range of significant contribution, $R$ covers a narrow value range $(\sim \pm 5 \%)$ I therefore substituted factorable centrold values in the attenuation and geometric terms $\bar{R}=065, \bar{R}=\sqrt{ } 14$ 
The Eigenfunctions are summarily defined in Appendix B. Again, $S_{\mathrm{e}}$ and $S_{\mathrm{h}}$ are not intrinsically normalized, so $E_{0}$ and $H_{0}$ do not indicate the true peak amplitudes. For the Local case, these functions turned out to be surprisingly similar to their Compton-dipole counterparts in form and magnitude, as illustrated by Fig. 3-8. Figure 3-9 depicts $S_{\mathrm{e}}$ and $S_{\mathrm{h}}$ for the Distant case.

The peak signal amplitudes are also indicated in those illustrations; obviously the Local signals should be comfortably visible $\left(0.3 \mathrm{~V} / \mathrm{m}\right.$ and $\left.0.005 \mathrm{~V} / \mathrm{m}^{2}\right)$. Figure $3-10$ depicts the $\dot{B}$ signals (Local and Distant). As calculated here, the Distant signals (at $5 \mathrm{~km}$ ) would be observationally marginal. They are surprisingly sharp-note the $10-\mu \mathrm{s} E$ crossover for $\sigma_{\mathrm{g}}=0.0025$. This feature seems exceptionally sensitive to earth conductivity; the $E$ crossover moves to $\sim 60 \mu$ s for $\sigma_{\mathrm{g}}=0.01$. Table 3-2 summarizes some quantitative signal features.

\subsection{Variations on the Theme}

In the course of investigation, I explored a variety of calculational procedures and physical models for the casing-current mode. For example, Fig. 3-10(a) shows a thick-band locus for $\dot{B}$; it indicates the spread of values calculated by three different computational schemes. Another variant was the so-called " $50-50$ mix," which consisted simply of an equipartition sum of the Local-slant computation and a range-scaled up-and-over contribution.* This generated even larger and sharper Local signals, as illustrated by Fig. 311. Figure 3-12 shows another incidental result-a typical calculated "anomalous $E_{\mathrm{z}}$ "一 the vertical $E$-field at ground surface and at $5 \mathrm{~km}$.

Figure 3-13 shows the "casing potential" for the three $\sigma_{\mathrm{g}}$ values. It represents the potential impulse that would be observed between the top of the casing (at SGZ) and "infinity"-i.e., a (low-resistance) ground probe some distance away $(\sim 200 \mathrm{~m})$. It was calculated assuming perfect discontinuity reflection, using a simpler convolution of the Sundé derivative.

Finally, there's the Diffusion Approximation; Figure 3-14 depicts the basic ideas. This calculation stays entirely in the time domain; its validity depends on the condition $\tau \gg \kappa \kappa_{0} / \sigma \approx 10^{-8} \mathrm{~s}$, where $\tau$ measures the time structure of the input impulse. (That is just satisfied within a few meters up the casing from DHZ.) Maxwell's equations then reduce to $\Delta^{2} H=h^{2} \partial H / \partial t$, where $h^{2}=\mu_{0} \sigma_{\mathrm{g}}=\pi \times 10^{-9}$. Taking account of geometry (and a few other incidentals), the induction signal from a step-function current element is the solution of

$\frac{\partial f}{\partial t}-2\left(\frac{1}{h r \sin \theta}\right)^{2} f=0$

Obviously, $f=e^{-2 \alpha t}$, where $\alpha=(h r \sin \theta)^{-2}$.

Fortuitously, the waveform parameter $(\alpha)$ is single-valued for all source points along the vertical line! ${ }^{\dagger}$ (That is, $r \sin \theta \equiv \rho$, a constant.) A source-point current function $f(t)$ can then be expressed at the sensor as a convolution of $H$-field step increments. The complete diffusion description is

$H_{\phi}=\sum \Delta H=h_{0} \frac{\Gamma}{2}\left[\sum_{1} I\left(x, t_{1}\right) G(x)\right](1-2 \alpha \delta t)$,

where $I$ is the Sundé (current) argument; $G$ describes the field distribution from a current element; the " $x$ " summation generates the casing-current distribution; the " 2 " summation adds the diffused fields; $t_{x}$ is defined indirectly by the Sundé variables; and $h_{0}$ is the "rabble" collection, as before $\left(h_{0}=112 \delta x \delta t\right)$.

Without further exploration of boring details, the outcome of this computation appears as Fig. 3-15. Considering the precarious basis for both calculations, the resemblance is almost embarrassingly good.

\footnotetext{
* Despite its empirical structure, the inital and final amplitude and phase convergences were exact for this model.

${ }^{\dagger}$ It is analogous to referencing Fourier phase to $\mathrm{DHZ}$, and it allows us to separate the time and space convolutions.
} 
Table 3-1. Fictitious casing-current-dipole parameters.

\begin{tabular}{|lrrrrc|}
\hline \multicolumn{1}{c}{$\sigma_{\mathrm{g}}$} & $\omega_{\mathrm{c}}$ & $Q$ & $L_{0}$ & $p_{0}$ & $a$ \\
\hline 0.0025 & 16000 & 0.036 & 2.6 & $0.24 \times 10^{6}$ & 80000 \\
0.005 & 8000 & 0.050 & 1.3 & $0.16 \times 10^{6}$ & 40000 \\
0.01 & 4000 & 0.065 & 0.64 & $0.10 \times 10^{6}$ & 20000 \\
\hline
\end{tabular}

\section{Table 3-2. Casing-current signal amplitudes and "cross-over" timings (X).}

\begin{tabular}{lccccccc|}
$\begin{array}{c}\sigma_{v} \\
(\mathrm{mho} / \mathrm{m})\end{array}$ & $\begin{array}{c}E \\
(\mathrm{~V} / \mathrm{m})\end{array}$ & $\begin{array}{c}X \\
(\mu \mathrm{s})\end{array}$ & $\begin{array}{c}H \\
(\Lambda \mathrm{t} / \mathrm{m})\end{array}$ & $\begin{array}{c}X \\
(\mu \mathrm{s})\end{array}$ & $\begin{array}{c}\dot{B}^{\prime} \\
\left(\mathrm{V} / \mathrm{m}^{2}\right)\end{array}$ & $\begin{array}{c}\dot{B}^{-} \\
\left(\mathrm{V} / \mathrm{m}^{2}\right)\end{array}$ & $\begin{array}{c}X \\
(\mu \mathrm{s})\end{array}$ \\
\hline Local & & & & & & & \\
\hline 0.0025 & 0.3 & 85 & 0.06 & $\sim 200$ & 0.005 & 0.0014 & 36 \\
0.005 & 0.1 & 170 & 0.036 & $\sim 400$ & 0.003 & 0.0008 & 72 \\
0.01 & 0.03 & 340 & 0.024 & $\sim 800$ & 0.002 & 0.0005 & 145 \\
Distant & & & & & & & \\
\hline 0.0025 & $2.2 \times 10^{-6}$ & 9.0 & $1.9 \times 10^{-7}$ & 14.5 & $8.2 \times 10^{-8}$ & $3.6 \times 10^{-8}$ & 7.0 \\
0.005 & $9.5 \times 10^{-8}$ & 21 & $18.5 \times 10^{-9}$ & 36 & $2.2 \times 10^{-8}$ & $8.0 \times 10^{-8}$ & 15 \\
0.01 & $4.6 \times 10^{-9}$ & 50 & $1.8 \times 10^{-9}$ & $\sim 95$ & $6.0 \times 10^{-9}$ & $2.0 \times 10^{-9}$ & 33.5 \\
\hline
\end{tabular}




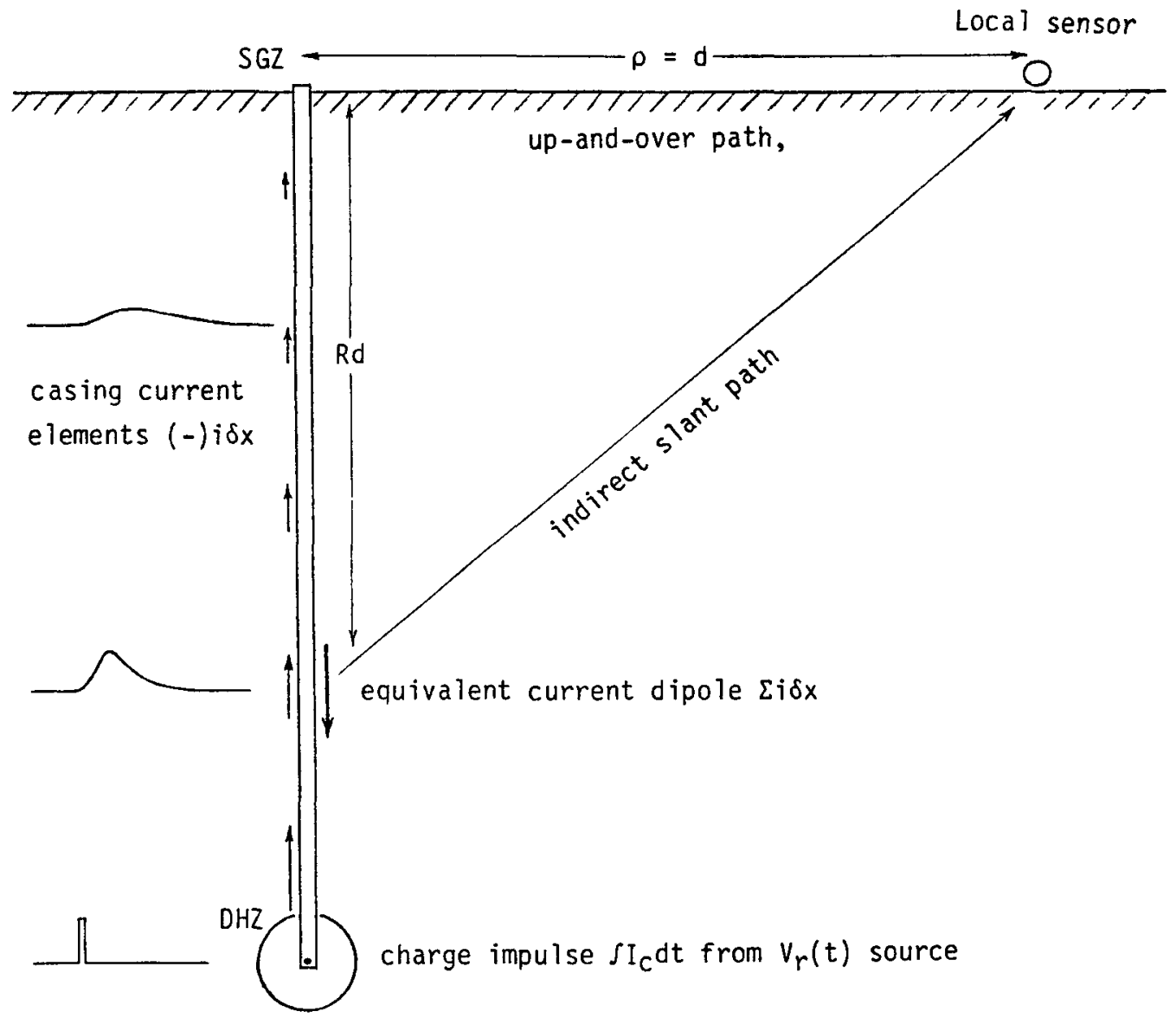

Figure 3-1. Casing-current representation (with fictitious dipole moment). 

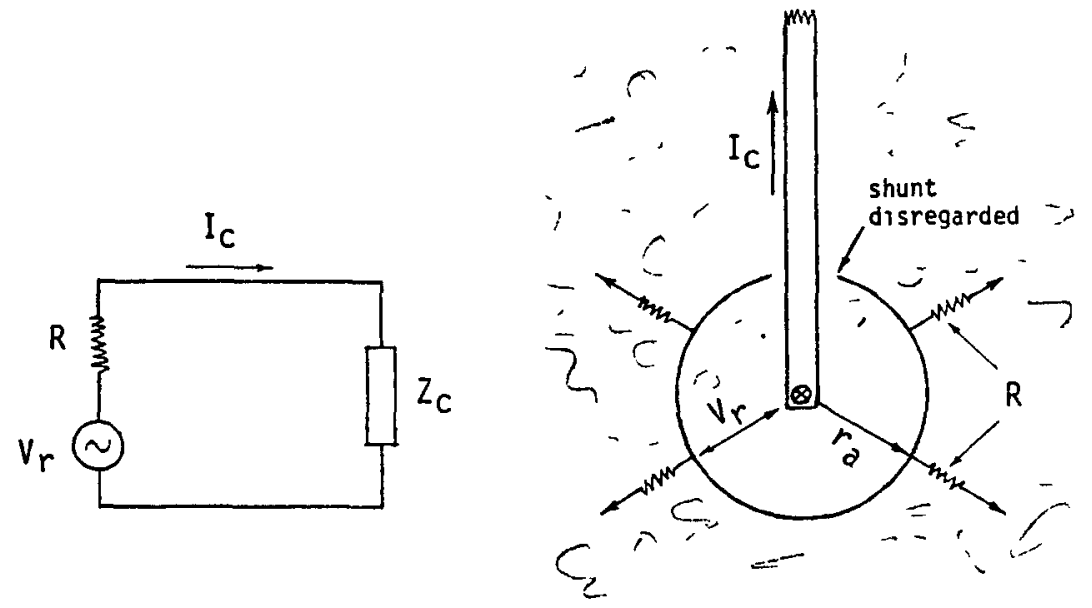

Figure 3-2. A simple equivalent-current model.

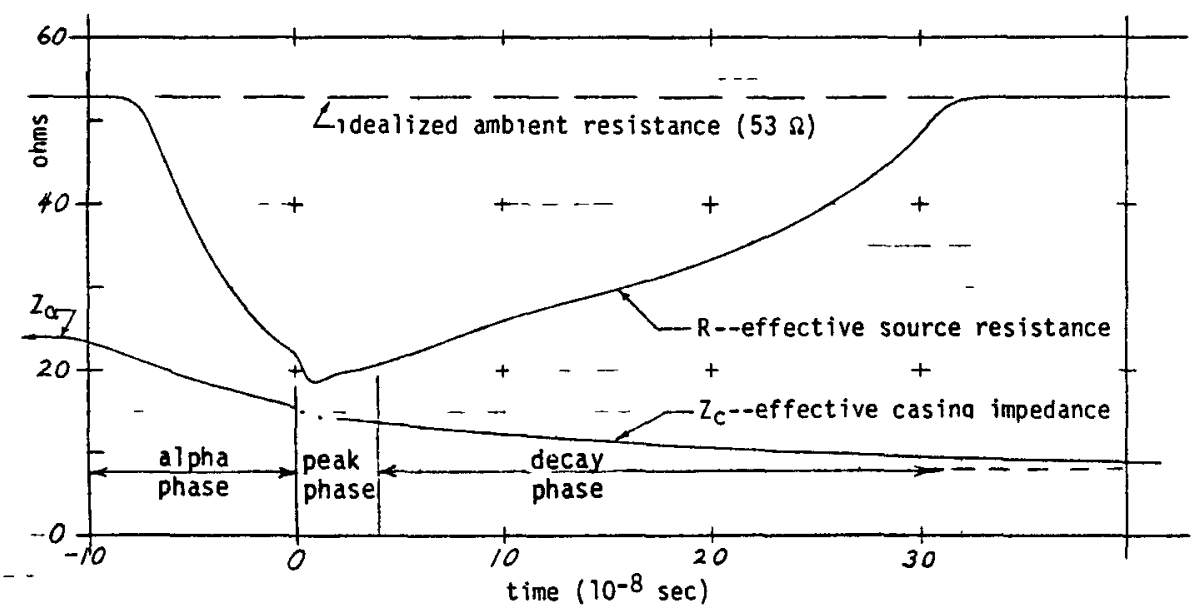

Figure 3-3. Equivalent-circuit parameters. 

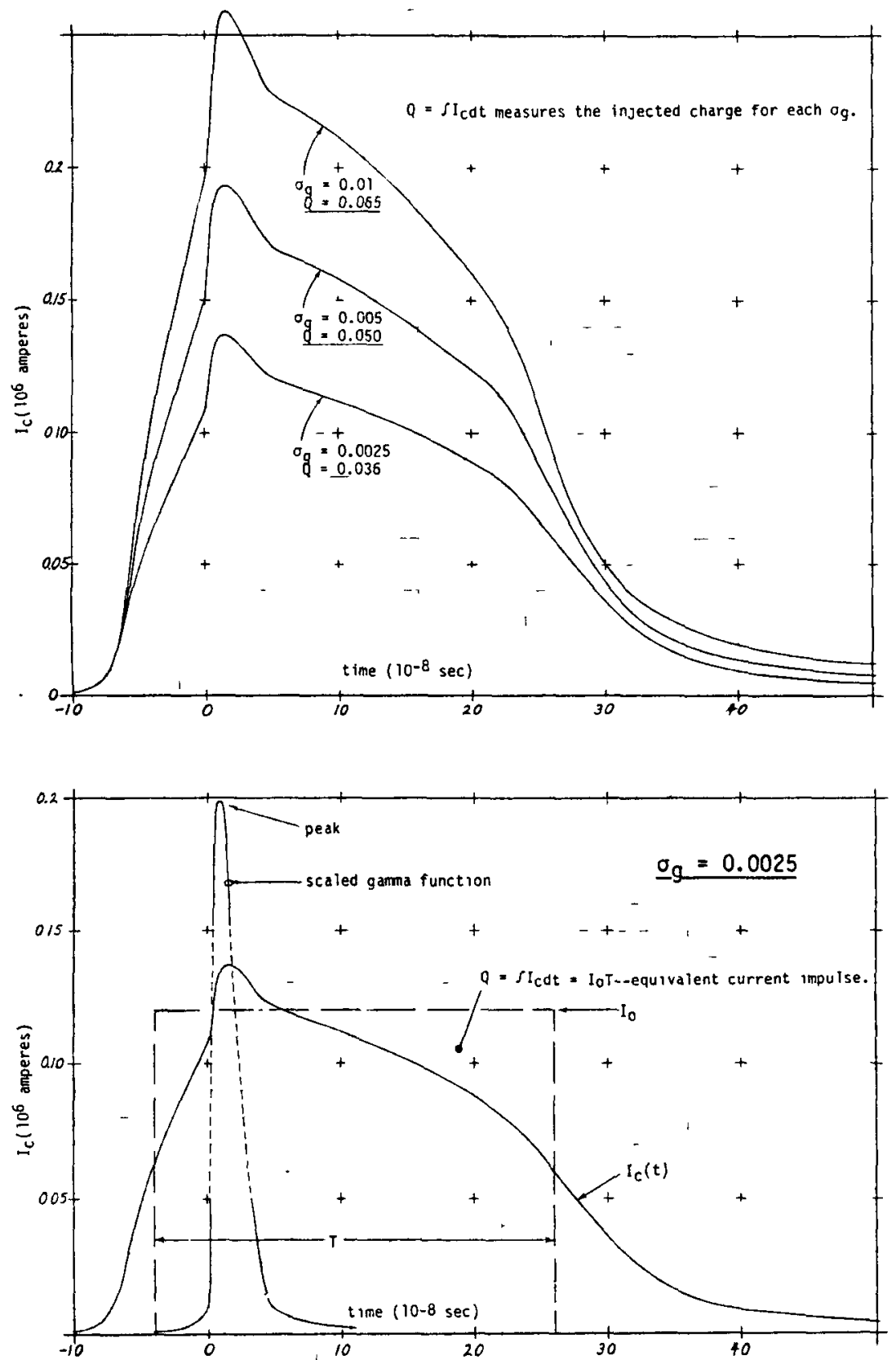

Figure 3-4. Casing-current input waveforms (at $\mathrm{DHZ}$ ). 


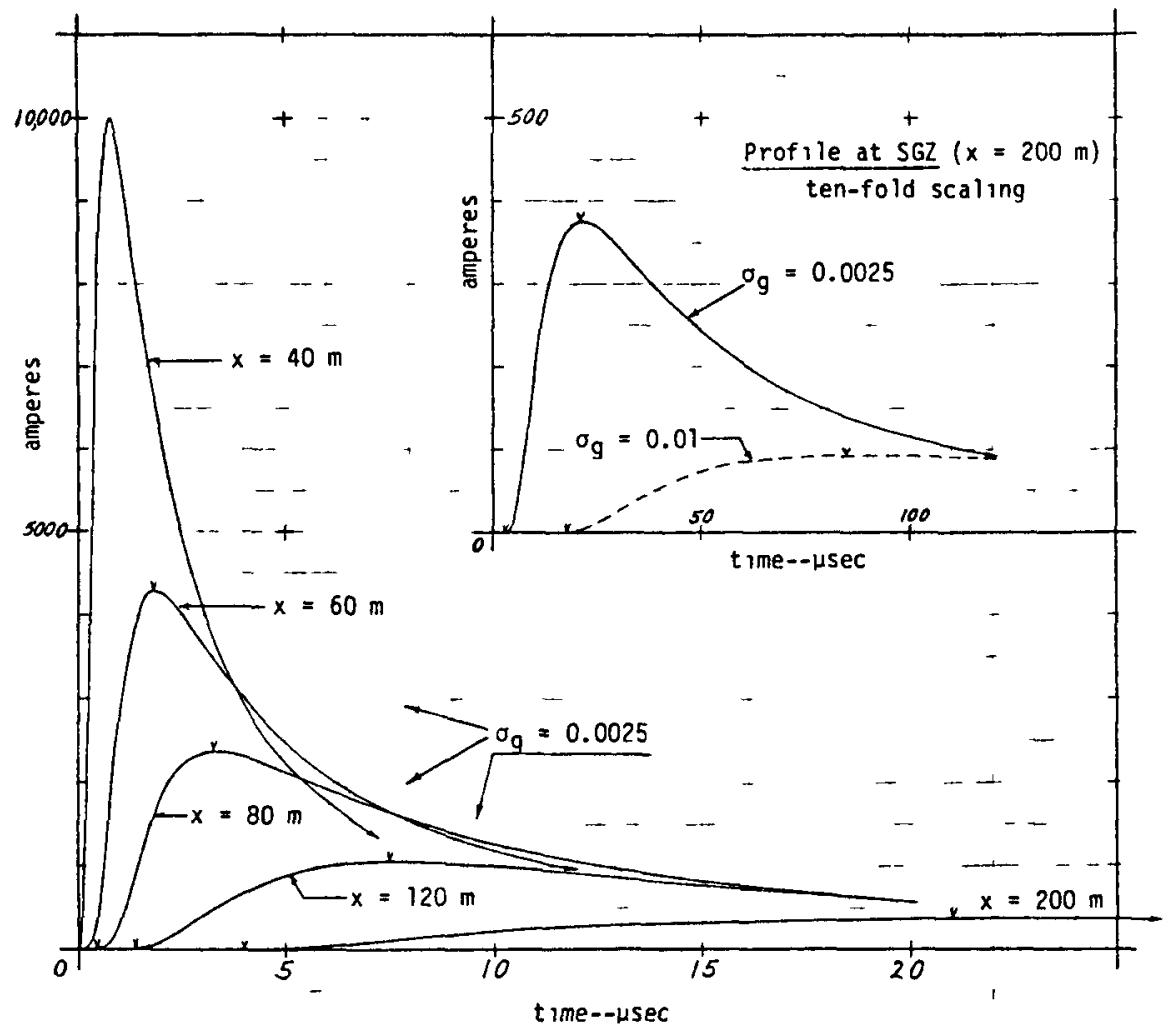

Figure 3-5. Casing-current profiles along casing; $\mathrm{Q}=.05$ coulomb input impulse. 

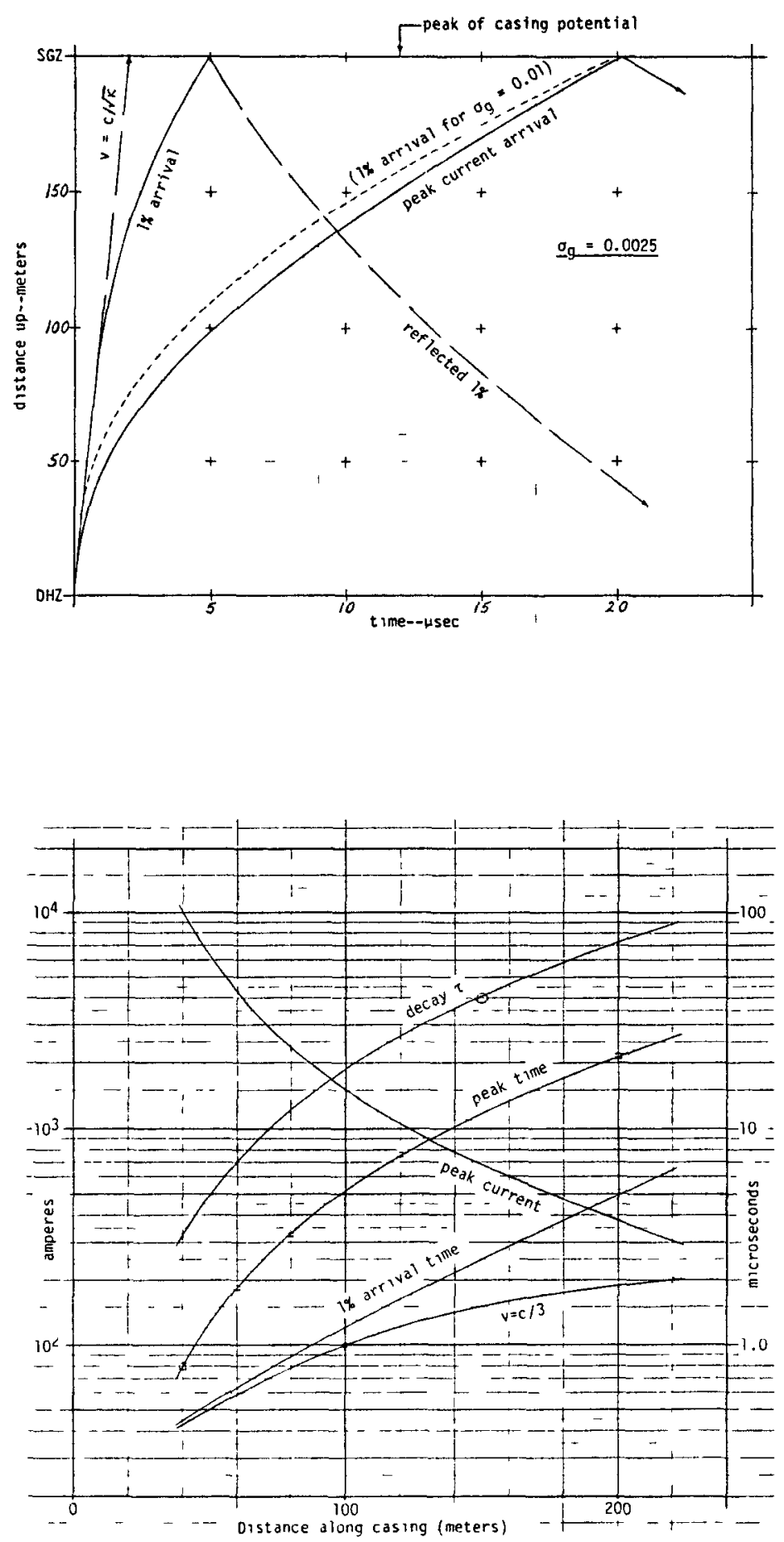

Figure 3-6. Some casing-current waveform features "going up." 


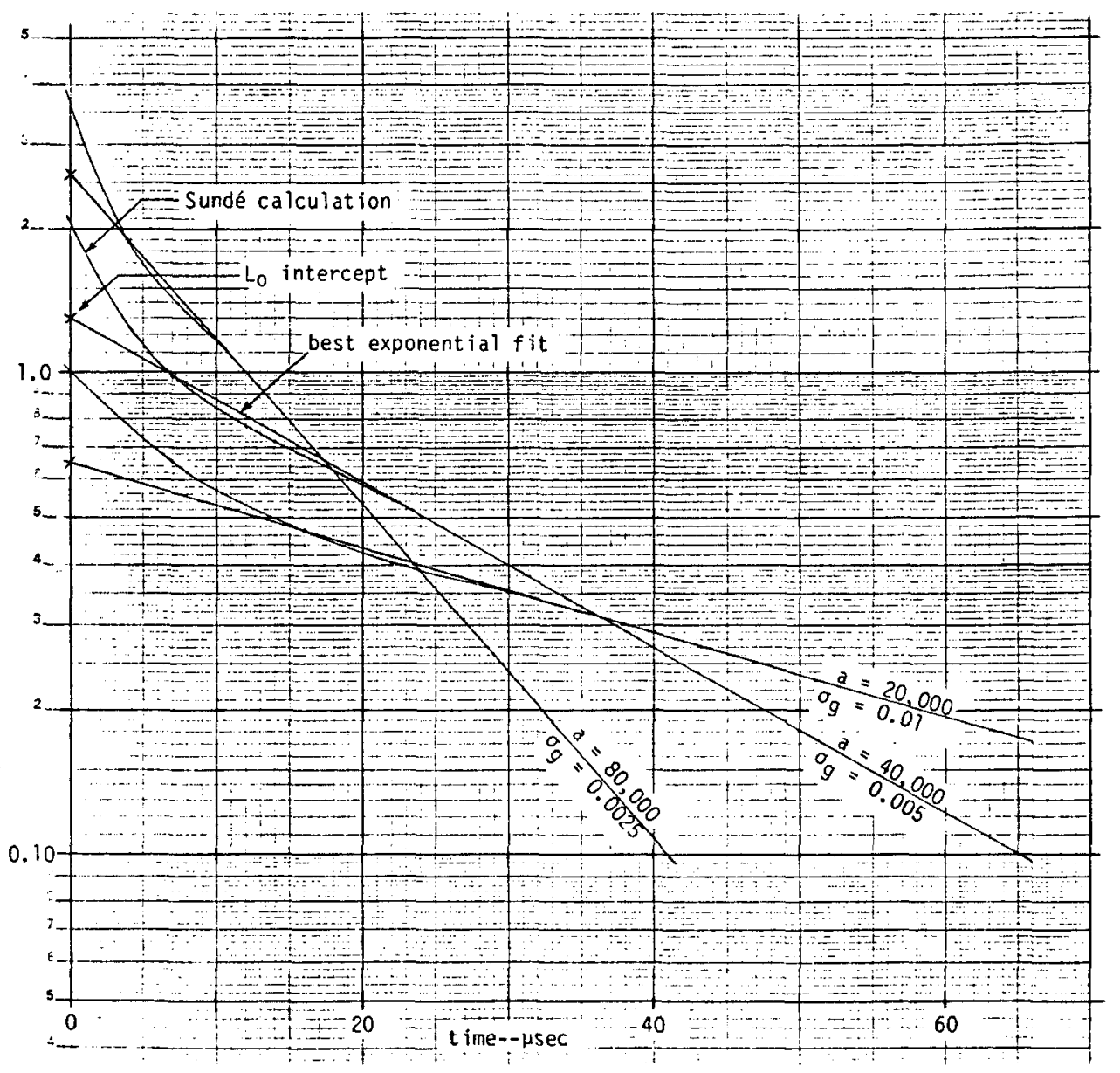

Figure 3-7. Fictitious casing-current dipole-moment histories-expressed as a dipole length parameter $L(t)$. 


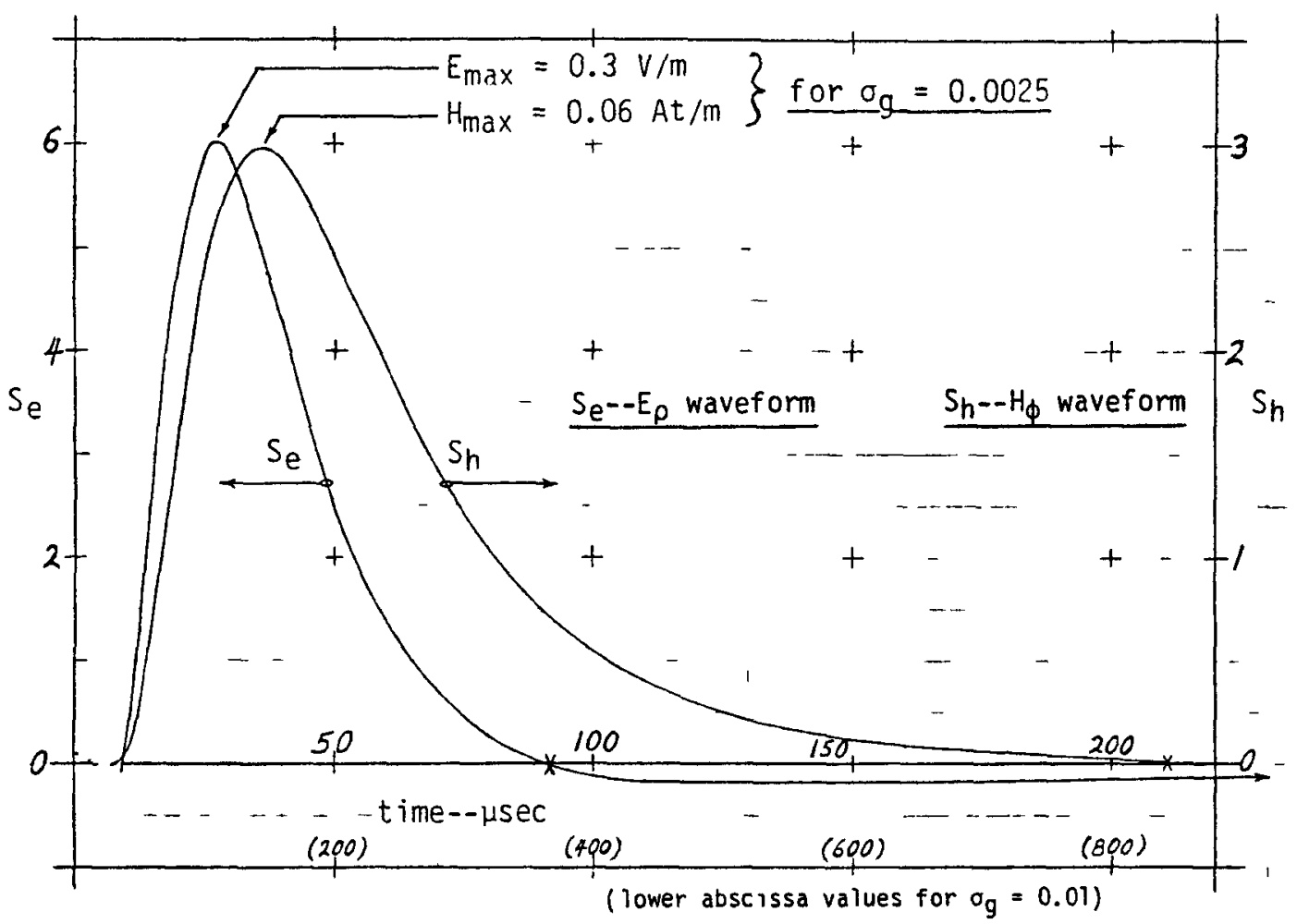

Figure 3-8. Local signal waveforms (IFT functions)-casing-current model. 


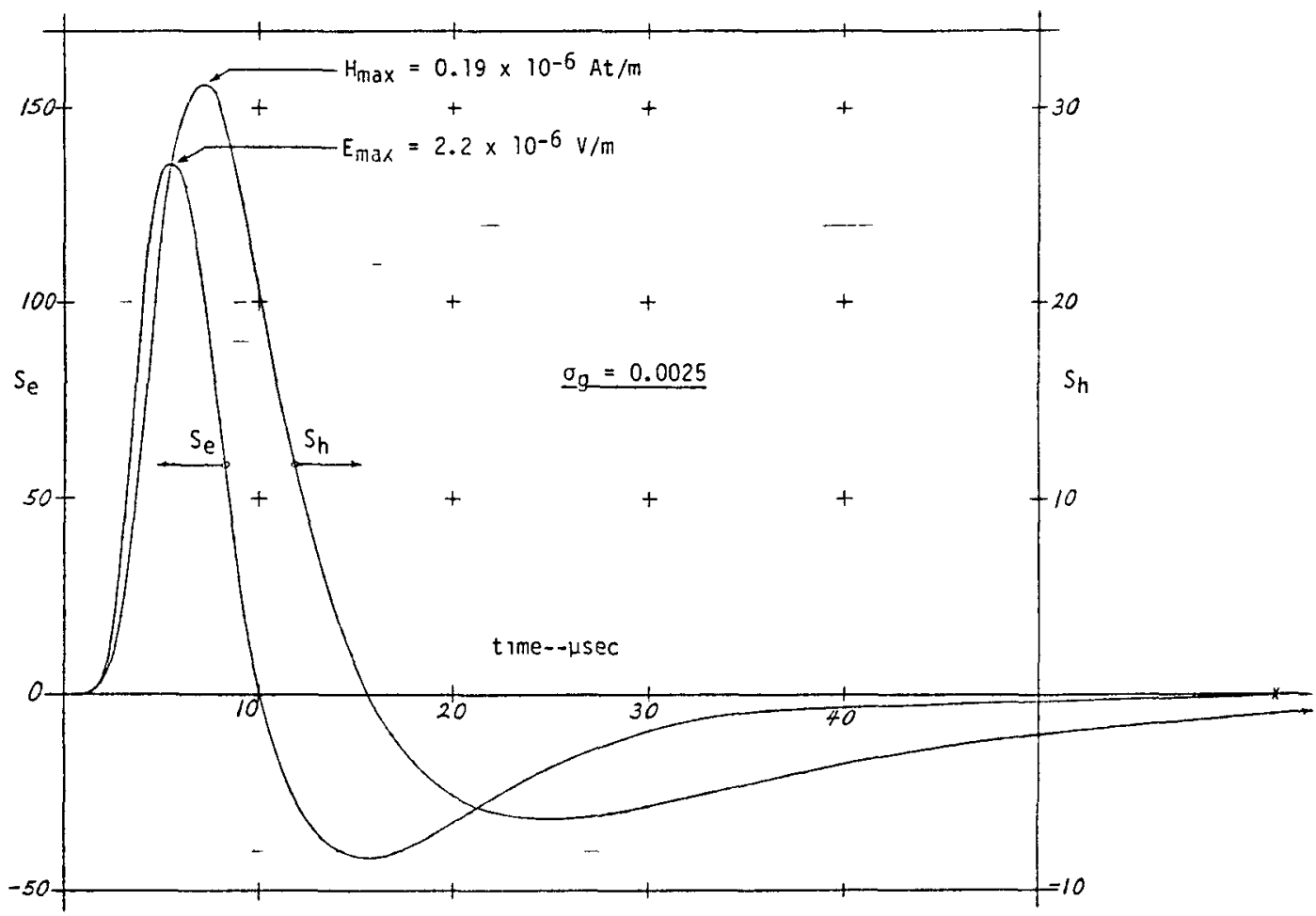

$S_{e^{-}-E_{\rho}}$ wave form $\quad S_{h}--H_{\Phi}$ waveform

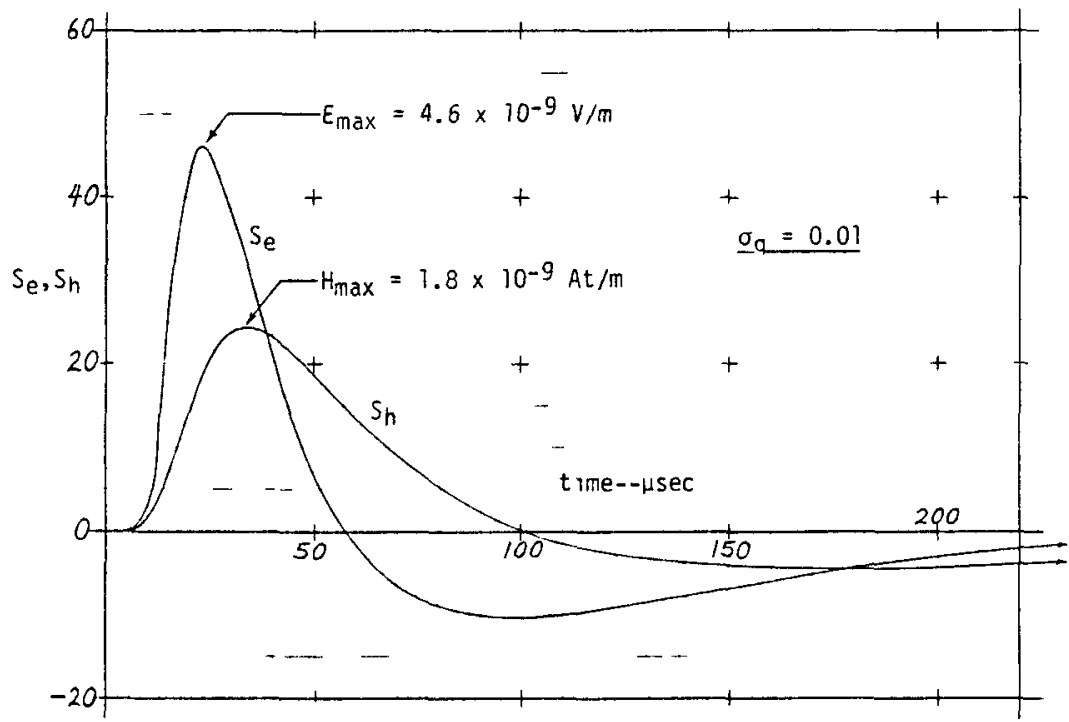

Figure 3-9. Distant signal waveforms (IFT functions)-casing-current model. 

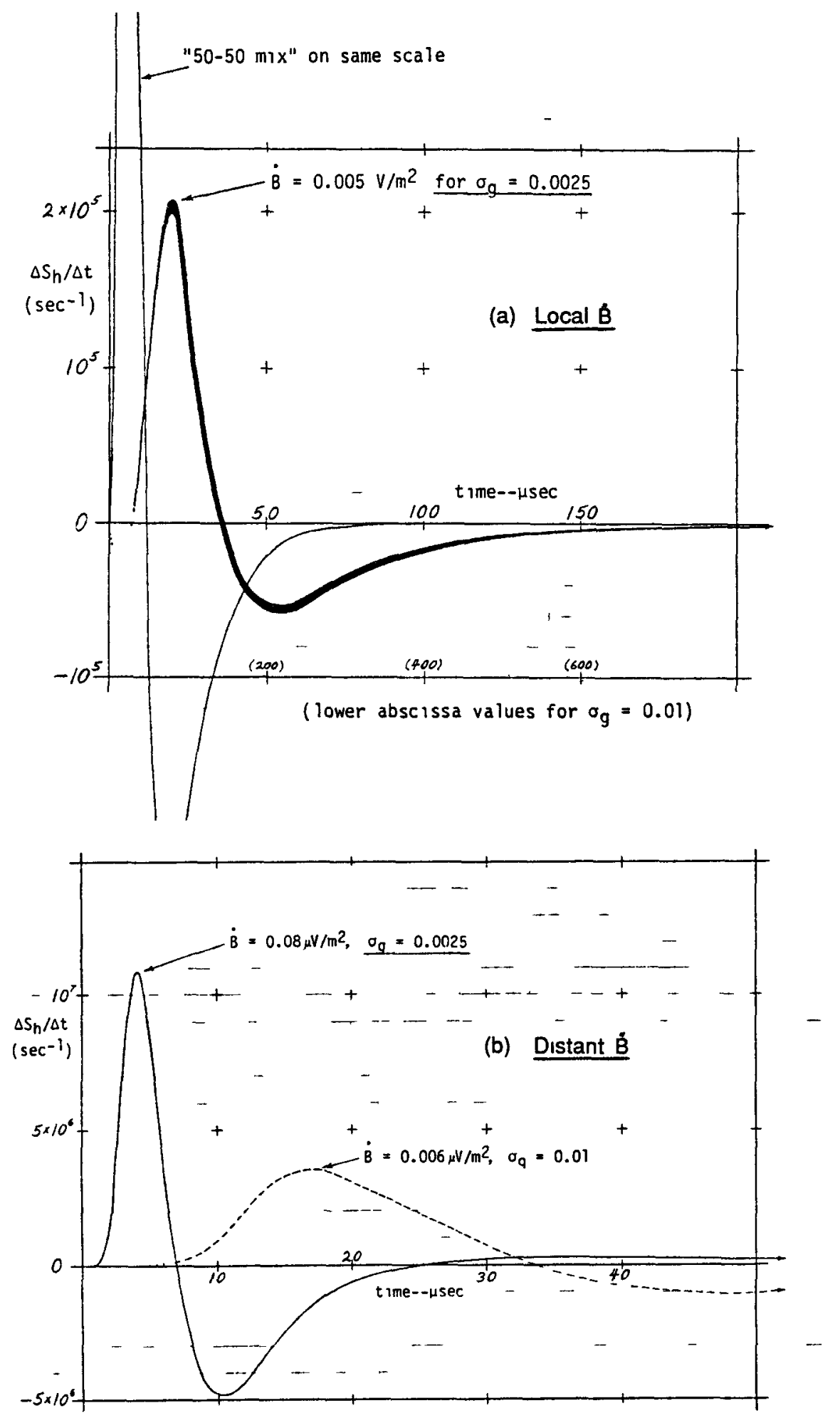

Figure 3-10. Local and distant B́ waveforms-casıng-current model. 

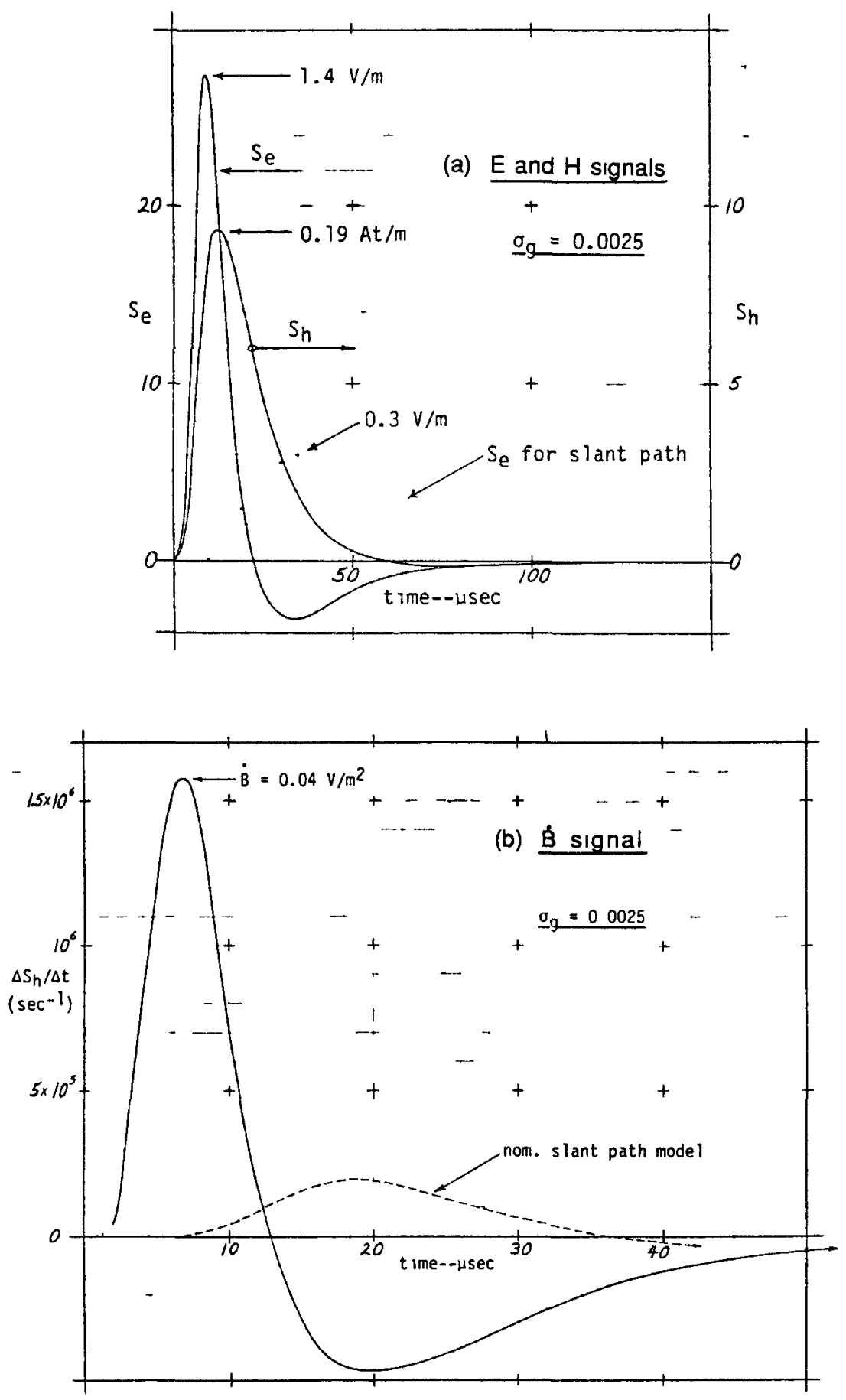

Figure 3-11. Local signal waveforms-"50-50 mix." 


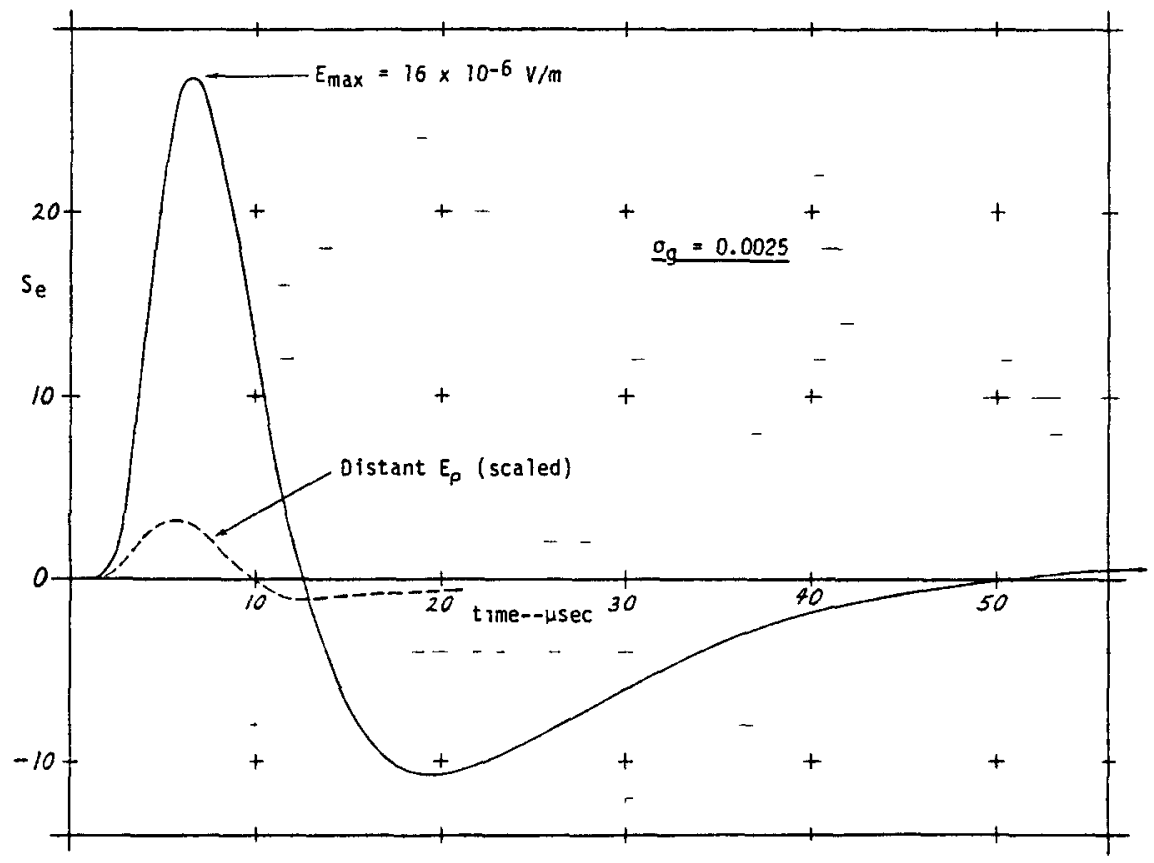

Figure 3-12. Distant waveform-anomalous $E_{z}$ mode.

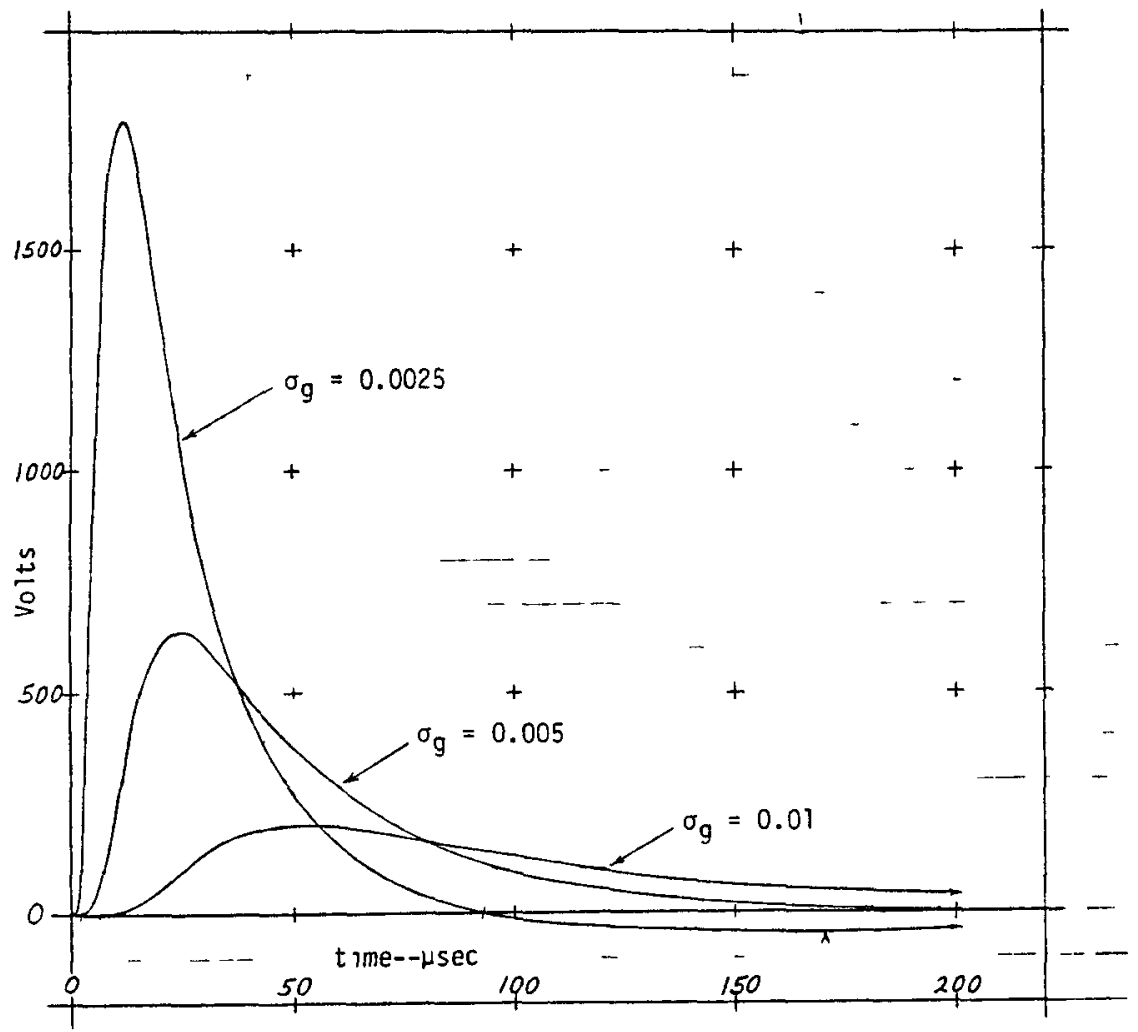

Figure 3-13. Casing potential waveforms (at SGZ). 

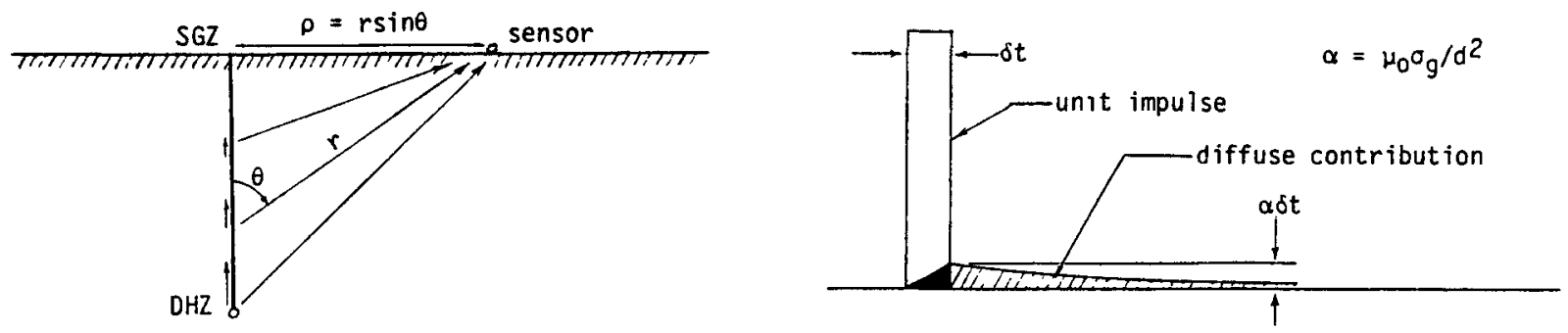

Figure 3-14. Diffusion approximation-basic concepts.

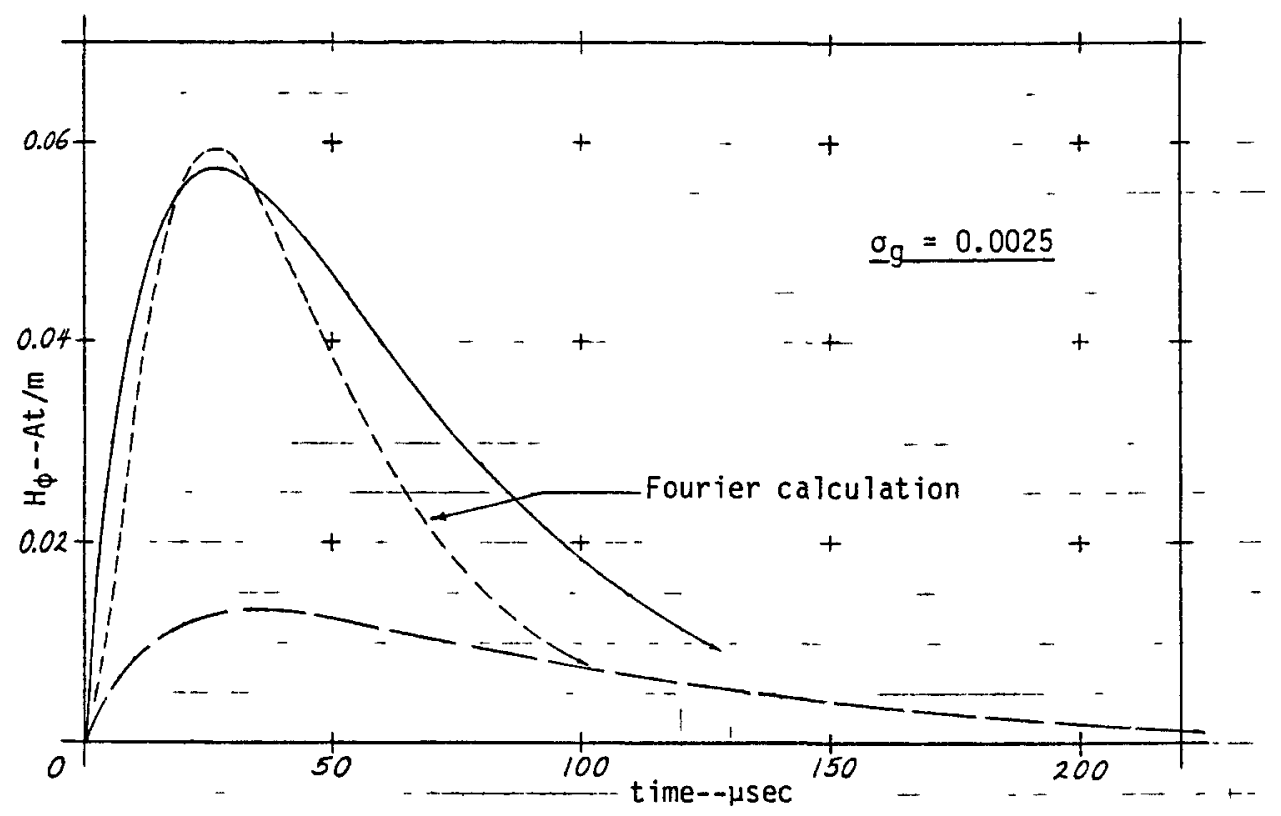

Figure 3-15. Local $\mathrm{H} \phi$ by diffusion approximation-casing-current model. 


\section{The Magnetic Bubble}

This model assumes that the highly conducting plasma in the early explosion cavity excludes the Earth's magnetic field as the cavity expands. ${ }^{7}$ As illustrated in Fig. 4-1, it behaves as a small diamagnetic sphere, to which I assigned an effective magnetic-dipole-moment history. This source definition involved three steps:

- Characterization of the history of cavity growth.

- Validation of a quasi-perfectly diamagnetic plasma.

- Expression of the dipole moment.

\subsection{Cavity History}

The analytic representation that I used for the cavity growth and "stabilization" was as follows:

$$
\begin{gathered}
n(t)=r_{\mathrm{c}}\left\lfloor 1+(x-1) e^{-\imath^{4}}\right\rfloor . \\
x=\left(r_{0} / r_{c}\right) W^{1 / 3}\left(t / W^{1 / 3}\right)^{2 / 5}=0.37(t / 2.15)^{0.4} \\
r_{\mathrm{c}}=R_{0}\left(\rho d_{0}\right)^{-1 / 4} W^{1 / 4}=32 \mathrm{~m}
\end{gathered}
$$

Time $(t)$ is in milliseconds, $W$ is the explosive yield in kilotons. The average density of earth is $\rho=$ $1.8 \mathrm{~g} / \mathrm{cm}^{3}$. The other parameters were normalized to the MERLIN data: $r_{0}=5.5 \mathrm{~m}, R_{0}=70 \mathrm{~m}, d_{0}=125 \mathrm{~m}$. This cavity history, $r(t)$, is depicted by Fig. 4-2.

The expansion of the ionized, highly-conducting cavity "gas" in the Earth's magnetic field induces a counter-current distribution in a plasma surface layer. Its thickness is measured by the EM depth of penetration: $\delta \approx 1 / \sqrt{ } \mu_{0} \sigma_{\mathrm{p}} \bar{\beta}$, where $\beta$ expresses the cavity expansion rate: $\beta \approx(1 / r)(\partial r / \partial t) \leq 200 \mathrm{~s}^{-1}$, and $\sigma_{\mathrm{p}}$ is the plasma conductivity: $\sigma_{\mathrm{p}}>\mathrm{Ne} \mu_{\mathrm{e}} \approx 2.5 \times 10^{4} \mathrm{mho} / \mathrm{m}$.

At or near cavity stabilization, the interior cavity temperature is still $\geq 10 \mathrm{eV}$. A primitive partition calculation showed that even then, the ionized gas is still multiply ionized. The value that I derived for $\sigma_{\mathrm{p}}$ is a lower bound, obtained by setting the free-electron density equal to the dissociated atomic density: $N_{\mathrm{e}} \approx N$. The electron mobility is $\mu_{\mathrm{e}} \approx(1 / 3)(e / m)(N \Sigma v)_{\mathrm{e}}^{-1} \approx 0.1 \mathrm{~m} / \mathrm{s}$ per $\mathrm{V} / \mathrm{m}$, using $\Sigma \approx 10^{-15} \mathrm{~cm}^{2}, N=$ $P V / k T \approx 3 \times 10^{18} \mathrm{~cm}^{-3} . P$ is the overburden pressure; $v$ and $T$ correspond to $10 \mathrm{eV}$. Finally, $\delta<0.4 \mathrm{~m}$ $\ll r_{c}$, so that we are justified in assuming that the expanding ionized cavity behaves like a quasi-perfect diamagnetic sphere.

\subsection{Magnetic Dipole Moment}

The distortion of the initially-uniform Earth's magnetic field by the diamagnetic "bubble," can be described as that due to an induced magnetic dipole of moment: $m(t)=(-) 2 \pi H_{\mathrm{e}} r^{3}{ }^{* 8}$ As $r \rightarrow r_{c}$, the asymptotic dipole strength becomes $\left|m_{0}\right|=2 \pi \mathrm{H}_{\mathrm{e}} r_{\mathrm{c}}^{3} \approx 8 \times 10^{6} \mathrm{~A} \cdot \mathrm{m}^{2}{ }^{+}$It is a strong EM source; the terminal energy content of the dipole induction field is about $150 \mathrm{~J}$.

\subsection{Sensor Signals}

At a sufficient distance $s \gg r_{\mathrm{c}^{\prime}}$ the observed induction signal due to that dipole moment is described by two orthogonal vector components: $h_{\rho}=h_{\mathrm{s}} f(t) \cos \psi$, and $h_{\theta}=(1 / 2) h_{\mathrm{s}} f(t) \sin \psi$, where $\psi$ is the azimuthal angle with respect to the Earth's field vector. $f(t)=\left(r / r_{c}\right)^{3}$, the signal time function; $h_{s}=H_{e}\left(r_{c} / s\right)^{3} \overline{A t}$, the amplitude coefficient. $\overline{A t}$ is a near-unity mean transmission that depends (weakly) on the choice of

\footnotetext{
${ }^{*} M_{1}=|(\mu-1) /(\mu+2)| 4 \pi H_{e} r^{3}$, where $\mu \rightarrow 0$.

'The induced moment vector necessarily opposes the Earth's field; hereafter I omit the polarity sign.
} 
propagation paths. The time function $f(t)$ is illustrated in Fig. 4-3 for our fiducial case, together with some possible restoring "tails" and a first-order half-sinusoid fitting function. Figures $4-4(a) /(b)$ illustrate the behavior of the dipole strength and time-structure as functions of yield, as obtained by scaling $r_{c}$.

The central tail in Fig. 4-3 corresponds to a relaxation of the Earth's field back into the explosion cavity for a time constant $\tau=\mu_{0} \sigma_{\mathrm{d}} r_{\mathrm{d}}^{2} \approx 0.03 \mathrm{~s} . r_{\mathrm{d}}$ is an intermediate shell thickness: $\delta<r_{\mathrm{d}}<r_{\mathrm{c}} . \sigma_{\mathrm{d}}$ is the late ionized-gas conductivity, which-following "recombination collapse"-becomes dominated by ionization due to the residual radioactivity in the cavity. By self-consistency, $\tau$ must be comparable to the mean period of (late) radioactive decay. That, in turn, is (approximately) the elapsed time to cavity stabilization.

The best-fitted half-sinusoid is a convenient and adequate analytic substitute: $\omega_{0}=20 \pi \mathrm{rad} / \mathrm{s}$ $(\sim 63 \mathrm{~Hz})$. Its Fourier transform produces significant components out to $u=3 \omega_{0}$, corresponding to about $200 \mathrm{~Hz}:$

$\mathrm{FT}_{\omega}=\left(2 / \omega_{0}\right)\left[1-\left(\omega / \omega_{0}\right)\right]^{-1} \cos \left(\frac{\pi \omega}{2 \omega_{0}}\right)$

(The apparent singularity at $\omega_{0}$ converges to a regular limit.)

Since $3 \omega_{0} \ll \omega_{\mathrm{c}} \approx 16000 \mathrm{rad} / \mathrm{s}$, the sensor waveform will look like the dipole time-function $f(t)$ (Fig. 4-3). This also allows us to contract the IFT entirely into an attenuation integral. The mean transmission factor $\overline{\mathrm{At}}$ was then defined as

$\overline{\mathrm{At}}=\frac{\int_{0}^{u} \mathrm{FT}_{\omega} \mathrm{At}_{\omega} e^{\jmath \omega t} d \omega}{\int_{0}^{u} \mathrm{FT}_{\omega} e^{\jmath \omega t} d \omega}$.

At ${ }_{\omega}$ is the Von Hippel attenuation: $\mathrm{At}_{\omega}=e^{-\beta \ell}$, where $\beta=\sqrt{\mu_{0} \sigma \omega / 2}$, and $\ell$ is the appropriate path length. Representative values for $\mathrm{At}_{\omega}$ and $\overline{\mathrm{At}}$ appear in Table 4-1.

At a Local sensor position $(\rho=d=200 \mathrm{~m})$, the peak signal can be expressed as $\Delta H=$ $h_{\mathrm{s}} \sqrt{\left(\cos ^{2} \psi\right)+\left(\sin ^{2} \psi\right) / 4}$. For our fiducial case, $h_{\mathrm{s}}=0.052 \mathrm{At} / \mathrm{m}$. (Here, $s$ is the slant-path length through the earth medium, in $h_{\mathrm{s}}$.) This magnetic signal was actually calculated in terms of three orthogonal $\Delta H$ components (with respect to a surface reference frame) at each of six "interesting" locations. The inclination of the Earth's field brings on a complexion of tedious factors in projective geometry that I will not further belabor here (values in range 0.1 to 1.0). Peak and vector-sum values are listed in Table 4-2, along with numbers for $\dot{B}$ and $E . \dot{B}$ was estimated using the elementary form: $\dot{B} \approx \mu_{0} \omega_{0}|\Delta H|{ }^{*}$ The $E$-field values were estimated using a Gaussian-integral approximation: $E \approx\left(\gamma^{2} d / 2 \sigma\right) /|\Delta H| \rightarrow(d / 2) \dot{B}$. Except for the smaller equatorial values, it is a surprisingly mild distribution. The entire array is presumed to follow the dipole time function $f(t)$. Figure 4-5 depicts two (normalized) sensor signal functions, with representative peak values. These are "visible" values. [The highest (on-axis) amplitudes are $8 \mu \mathrm{V} / \mathrm{m}^{2}$ and $800 \mu \mathrm{V} / \mathrm{m}$.]

For the Distant-sensor cases $(\rho=5 \mathrm{~km})$, I substituted a fictitious (and vector-parallel) dipole moment at the surface (as developed by Wait and Keller). Now the attenuation path is "straight up," such that the effective dipole moment strength becomes $m_{0}^{*}=\overline{\mathrm{At}}{ }^{*} m_{0} \approx 7 \times 10^{6} \mathrm{At} \cdot \mathrm{m}^{2}$. I applied the Kraichman-Zablocki formalisms to its vertical and horizontal components (as $m_{z}=m_{0}^{*} \cos 30^{\circ}, m_{\rho}=m_{0}^{*} \sin 30^{\circ}$ ) to calculate the Distant surface fields. ${ }^{\$ 3,4}$ (The propagation factor $|\gamma \rho|$ appears in higher order, for which I entered the values at $\omega_{0}$. Otherwise, the $\mathrm{VH}$ factor is essentially unity over ground surface.) These calculations obtained an almost flat vector-sum distribution (around the vertical LOS axis) for the magnetic signals: $\bar{\Delta} \bar{H} \approx 22 \times 10^{-6} \mathrm{At} / \mathrm{m}$. As before, peak $\dot{B} \approx \mu_{0} \omega_{0} \bar{\Delta} \bar{H}=2 \times 10^{-9} \mathrm{~V} / \mathrm{m}^{2}$. I estimated $E$ from induced earth currents, using an intrinsic-impedance formalism: $E=\mid \gamma / \sigma H \rightarrow \sqrt{\mu_{0} \omega_{0}} / \sigma_{\mathrm{g}} \Delta H=0.18 \Delta H \approx 4 \mu \mathrm{V} / \mathrm{m}$. The waveforms resemble Fig. 4-5; these are marginally visible signal values, at best.

\footnotetext{
* The rate multiplier turns out to be close to $\omega_{0}$, however the problem is approached.

${ }^{\dagger}$ Asymptotic VMD components: $H_{0}^{z}=\left(m_{z} / 2 \pi \rho^{3}\right) ; H_{z}^{v}=\left(9|\gamma \rho|^{2}\right) H_{0}^{\mathrm{v}} ; H_{\rho}^{\mathrm{v}}=(4 /|\gamma \rho|) H_{0}^{z}$.

Asymptotic HMD components: $H_{0}^{\rho}=\left(m_{\rho} / 2 \pi \rho^{3}\right)=H_{\phi}^{\mathrm{h}}(\mathrm{E}-\mathrm{W}) ; H_{z}^{\mathrm{h}}=\left(-4|\gamma \rho|^{2}\right) H_{0}^{\rho} ; H_{\rho}^{\mathrm{h}}=2 H_{0}^{\rho}(\mathrm{N}-\mathrm{S})$.
} 
Table 4-1. EM transmissions in the magnetic-bubble domain.

\begin{tabular}{|lllll|}
\hline \multicolumn{1}{|c}{$\sigma_{\mathrm{g}}$} & $\omega_{0} / 3$ & $\omega_{0}$ & $3 \omega_{0}$ & $\overline{\mathrm{At}}$ \\
\hline Vertical path $(r=d)$ & & & & \\
\hline 0.01 & 0.903 & 0.882 & 0.805 & 0.89 \\
0.0025 & 0.964 & 0.939 & 0.897 & 0.94 \\
Slant path $(r / d=\sqrt{2})$ & & & & \\
\hline 0.01 & 0.903 & 0.838 & 0.736 & 0.85 \\
0.0025 & 0.950 & 0.915 & 0.858 & 0.92 \\
\hline
\end{tabular}

Table 4-2. Magnetic-bubble local-signal amplitudes. ${ }^{a}$

\begin{tabular}{|llccccc|}
\hline Location & $\begin{array}{c}\Delta H_{z} \\
(\mathrm{At} / \mathrm{m})\end{array}$ & $\begin{array}{c}\Delta H_{p} \\
(\mathrm{At} / \mathrm{m})\end{array}$ & $\begin{array}{c}\Delta H_{\phi} \\
(\mathrm{At} / \mathrm{m})\end{array}$ & $\begin{array}{c}\Delta H \\
(\mathrm{At} / \mathrm{m})\end{array}$ & $\begin{array}{c}\dot{B} \\
\left(\mu \mathrm{V} / \mathrm{m}^{2}\right)\end{array}$ & $\begin{array}{c}E \\
(\mu \mathrm{V} / \mathrm{m})\end{array}$ \\
\hline North & 0.0087 & 0.029 & 0 & 0.0305 & 2.4 & 240 \\
East & 0.0098 & 0.038 & 0.0087 & 0.040 & 3.15 & 315 \\
South & 0.033 & 0.044 & 0 & 0.055 & 4.3 & 430 \\
West & 0.0098 & 0.038 & 0.0087 & 0.040 & 3.15 & 315 \\
On-axis (south) & 0.088 & 0.051 & 0 & 0.102 & 8.0 & 800 \\
Equatorial (north) & 0.0050 & 0.0085 & 0 & 0.010 & 0.78 & 78 \\
\hline ambient $H_{\mathrm{e}} \approx \mathbf{4 0} \mathrm{At} / \mathrm{m}$. & & & & & & \\
\hline
\end{tabular}




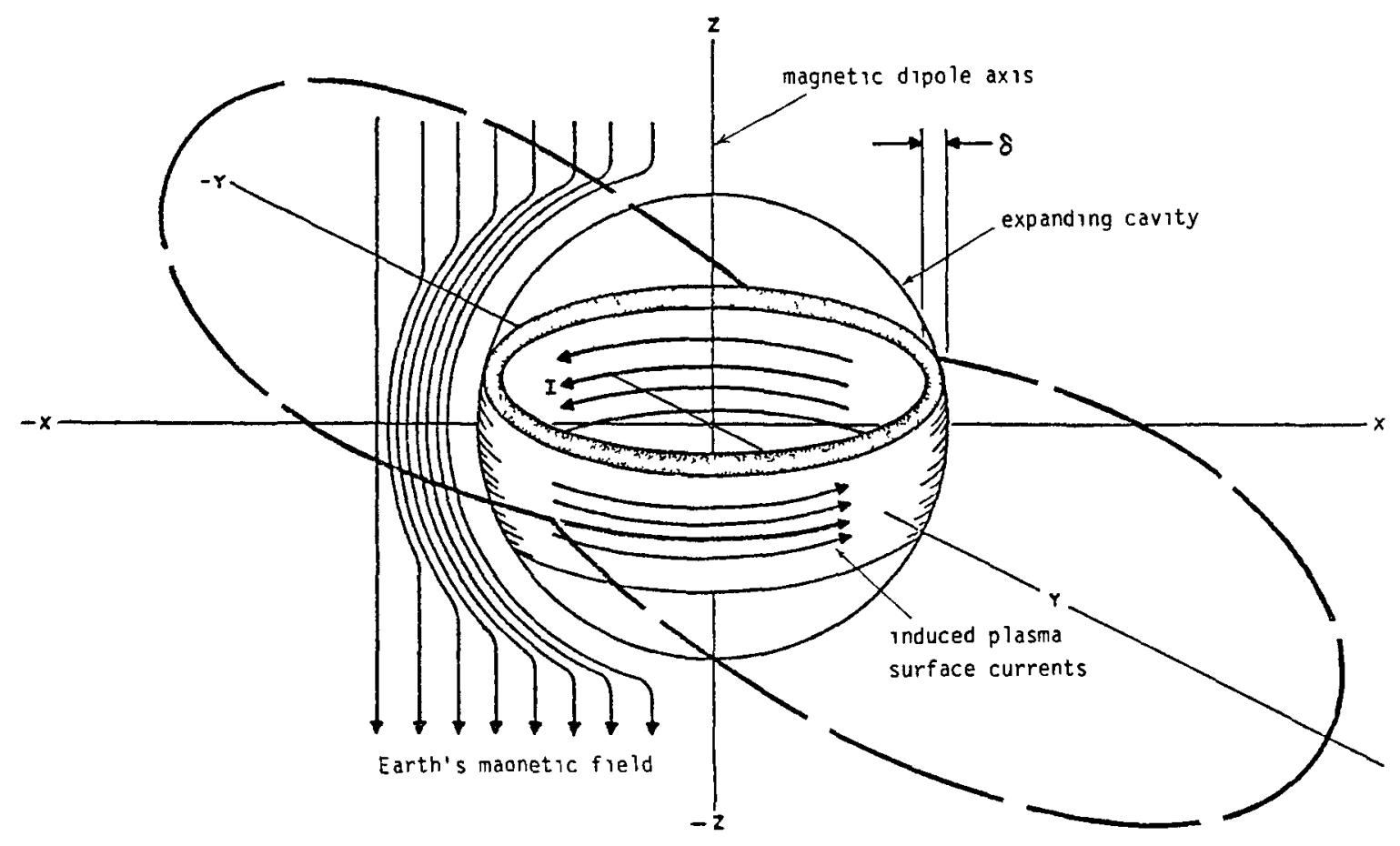

Figure 4-1. The magnetic bubble.

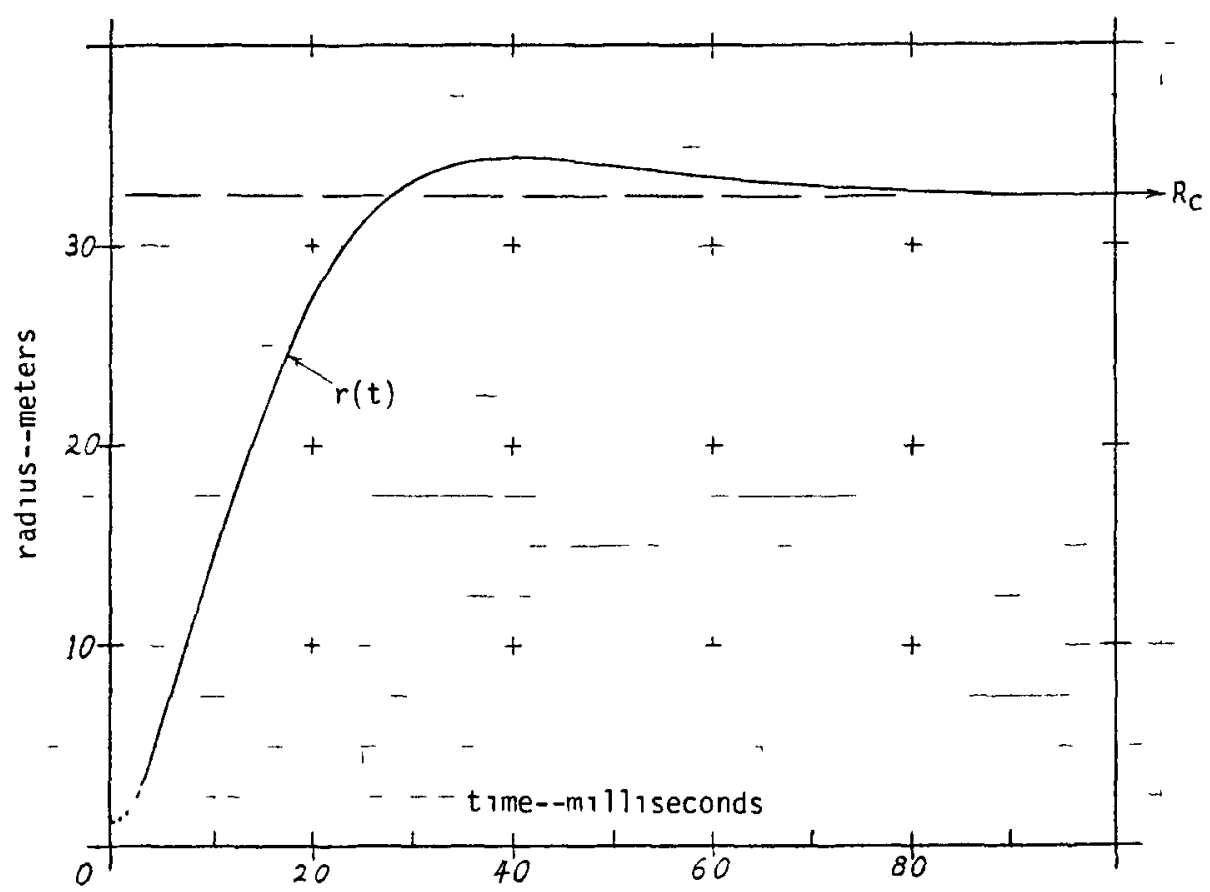

Figure 4-2. Assumed cavity-growth history. 


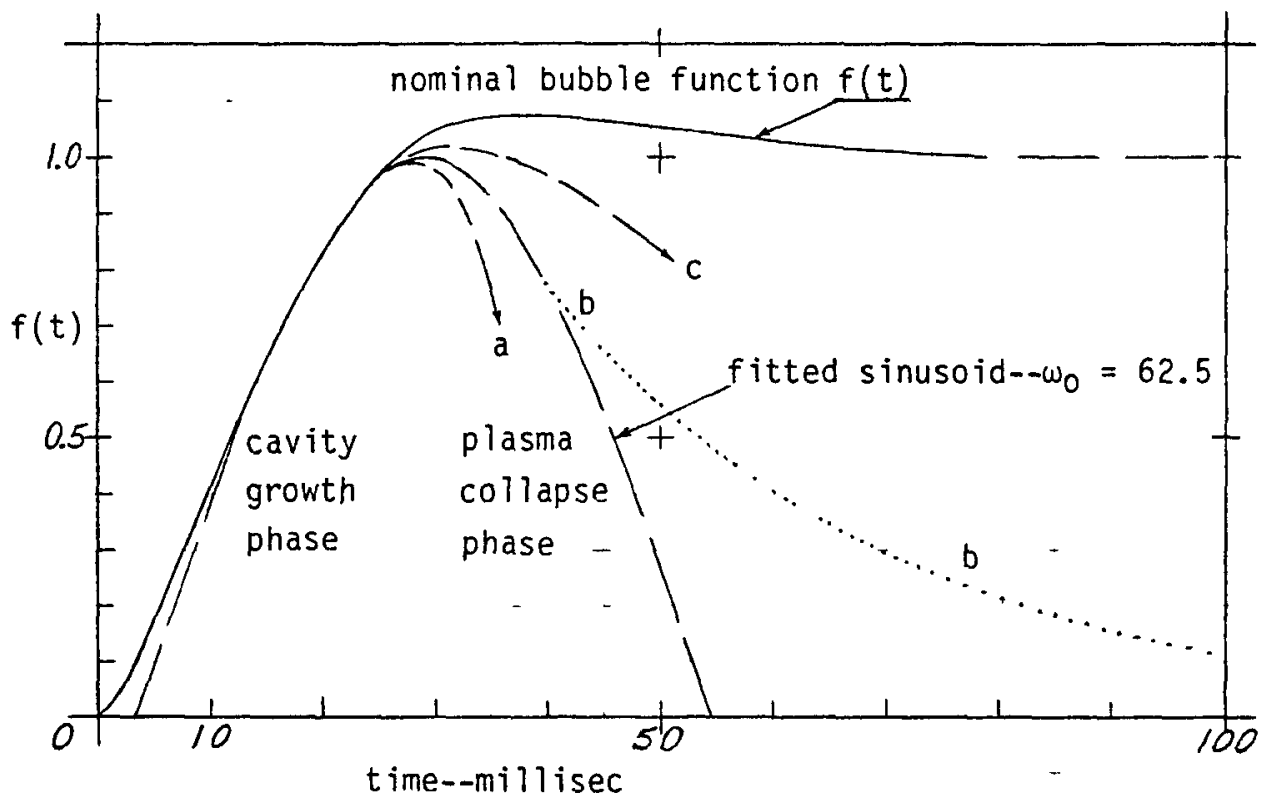

Figure 4-3. Magnetic-bubble dipole-moment history.
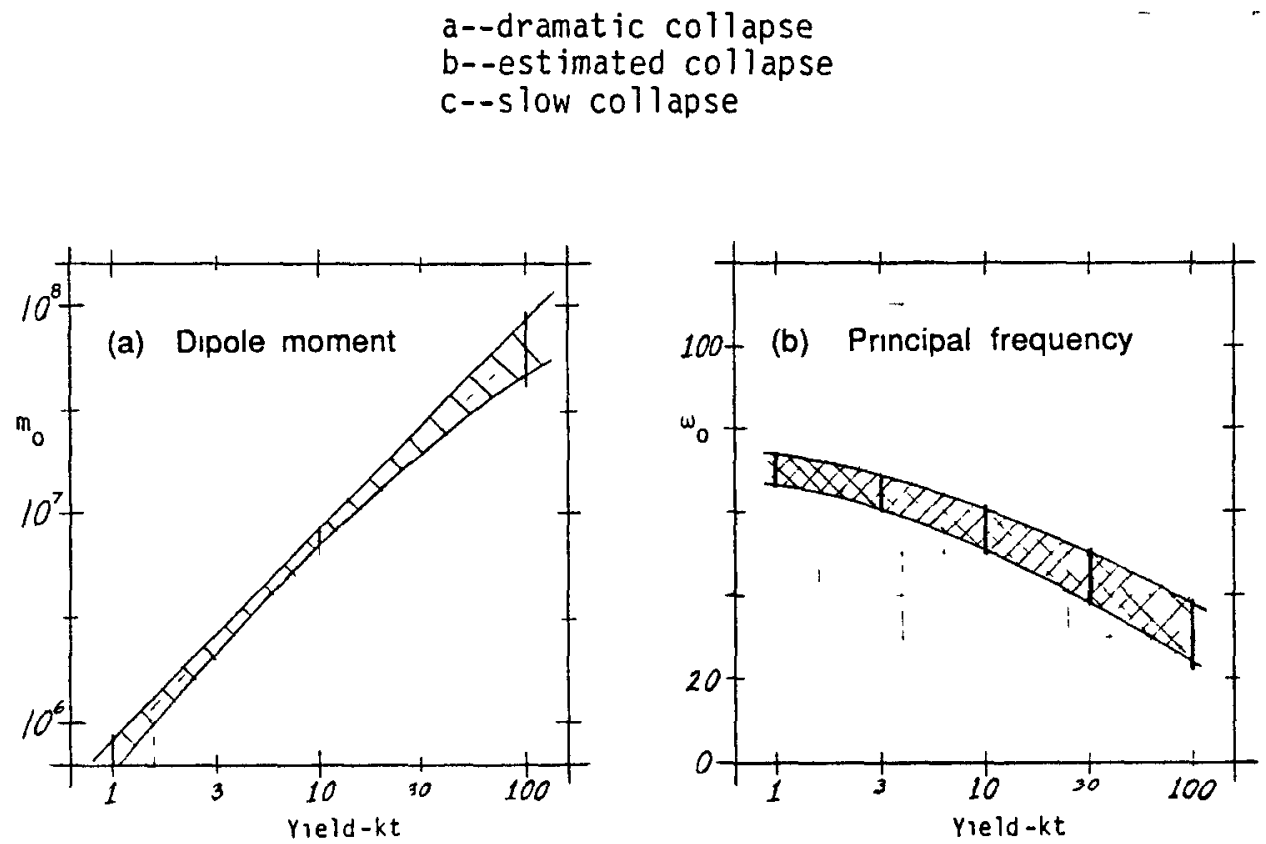

Figure 4-4. Magnetic-bubble dipole features as functions of yield. 


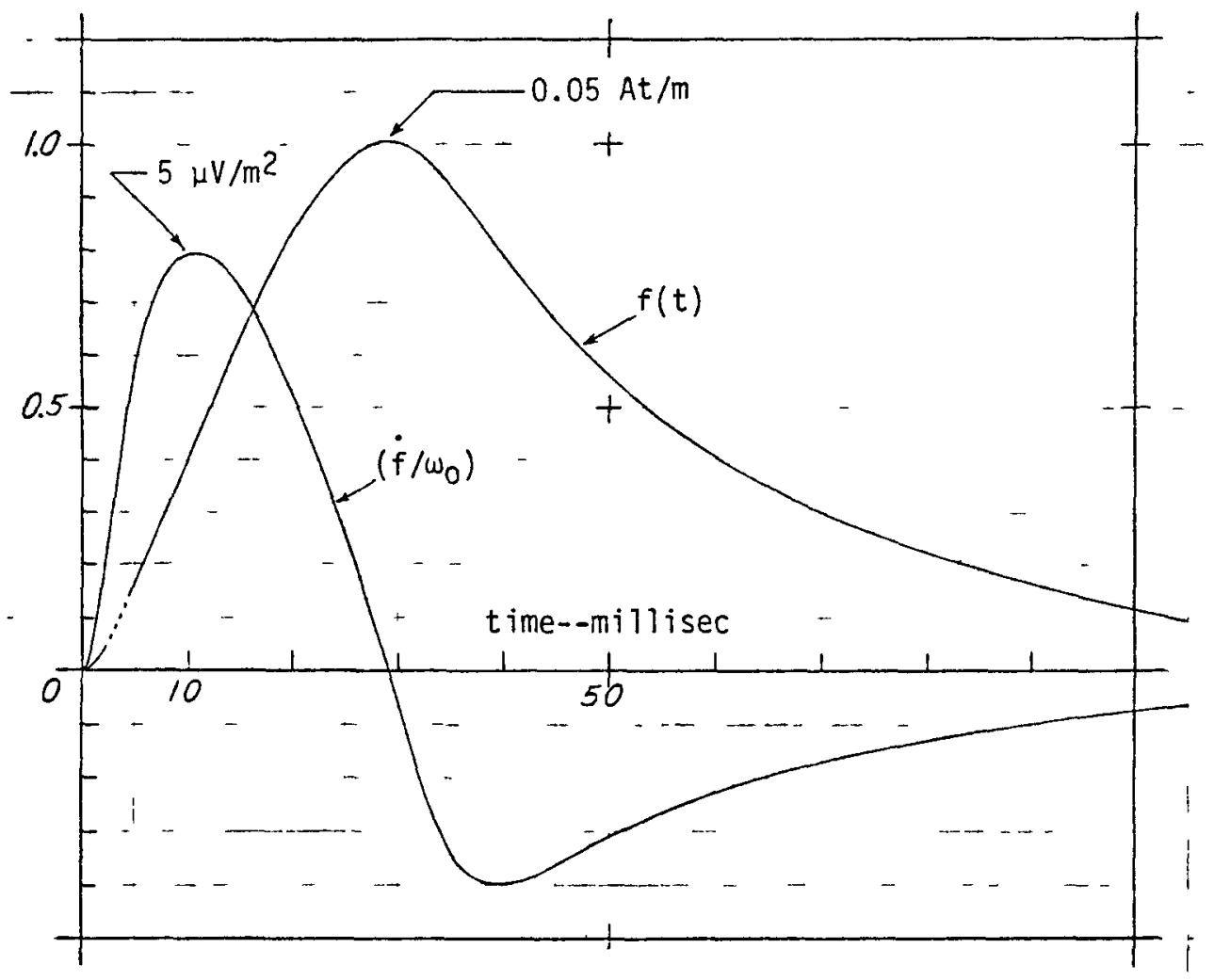

Figure 4-5. Magnetic-bubble signal waveforms. (Local values.) 


\section{The Magneto-Acoustic Wave}

The operative mechanism here is the interception of the Earth's magnetic field by the large-scale ground-motion or "acoustic wave" that follows cavity expansion. It generates an expanding, azimuthal, spherical-shell current distribution by Faraday induction, as depicted in Fig. 5-1. To evaluate this model, I calculated the surrogate magnetic-dipole moment for the infinite-medium case. It obtains extremely small signal predictions as an upper-limit bound for the real, ground-surface case.

\subsection{Magnetic Dipole Moment}

This calculation integrates the contributions from the "bicycle tire" elements that comprise the current shell. This involves three steps:

- Definition of the Faraday current elements.

- Characterization of the particle motion of the loop elements.

- Expression of the dipole moment.

The induced emf around a loop element must satisfy the circuital integral; that obtains $E_{\phi}=$ $|\dot{r} \times B|=r \dot{B}_{\mathrm{e}} \sin \theta$. For the fiducial example at $r=d(\mathrm{dob}): r \approx 2 \mathrm{~m} / \mathrm{s}$, whence $E_{\phi} \approx 10^{-4} \mathrm{~V} / \mathrm{m}$ (max). The corresponding emf around an equatorial loop is $V=\pi d E_{\phi} \approx 0.06 \mathrm{~V}$, and the peak induced-current density in the earth medium is $J_{\phi}=\sigma_{\mathrm{g}} E_{\phi} \approx 0.5 \mu \mathrm{A} / \mathrm{m}^{2}$. The dipole-moment contribution from a particular loop element is $\delta m=\delta i A_{r}=J_{\phi} \delta a A_{r} .\left(\delta a, A_{r}\right.$ are indicated in Fig. 5-1.)

The moment integral must sum over $r$ as well as $\theta$, because the instantaneous current shell includes the (thick) radial space of significant particle-velocity distribution $r(r)$. The local ground-motion velocity $\left(\dot{r}_{\mathrm{m}} \approx 1\right.$ to $\left.10 \mathrm{~m} / \mathrm{s}\right)$ is much less than the acoustic velocity $\left(v_{\mathrm{s}} \approx 2000 \mathrm{~m} / \mathrm{s}\right)$. I can then separately specify the local $r(t)$ function and the peak-amplitude distribution as $\dot{r}=r_{\mathrm{m}} f\left(t^{*}\right)$, and $r_{\mathrm{m}}=\dot{r}_{\mathrm{c}}\left(r / r_{\mathrm{c}}\right)^{-10 / 3}$, where $t^{*}$ is the relative time given by $t^{*}=t-r / v_{\mathrm{s}}=t-\left(r / r_{\mathrm{c}}\right) \tau^{*}$ and $r_{\mathrm{c}}$ is a fictitious initial particle velocity referenced back to the final cavity radius. The instantaneous particle-velocity history is described by

$f\left(t^{*}\right)=\sin \left(\omega_{0} t^{*}\right) e^{-\gamma t^{*}}$

as illustrated by Fig. 5-2. In this equation, $\omega_{0}$ is a "preferred frequency" related to the acoustic velocity and the shear modulus of the medium. ( $\gamma=\omega_{0} / 2 \pi, \omega_{0} / \pi, 2 \omega_{0} / \pi$-not critical.)

The dipole-moment integral becomes

$m=\iint_{1} \delta m=\frac{4 \pi}{3} \sigma_{\mathrm{g}} B_{\mathrm{e}} \int\left(\dot{r} r^{3}\right) d r=m_{\mathrm{c}} F_{0}(t)$

where

$m_{\mathrm{s}}=V_{\mathrm{c}} h_{\mathrm{c}}=\left(\frac{4 \pi}{3} r_{\mathrm{r}}^{3}\right)\left(\sigma_{\mathrm{g}} B_{\mathrm{a}} r_{\mathrm{c}} r_{\mathrm{r}}\right)$

and

$F_{0}(t)=\sum_{r_{\mathrm{c}}}^{v_{\mathrm{b}} t}\left(r_{\mathrm{c}} / r\right)^{1 / 3} \sin \left(\omega_{0} t^{*}\right) e^{-\gamma \gamma^{*}}\left(\delta r / r_{\mathrm{c}}\right)$

As before, the ground-motion coefficient values were chosen to fit the MERLIN data: $r_{\mathrm{c}}=32 \mathrm{~m}$, $\dot{r}_{\mathrm{c}}=175 \mathrm{~m} / \mathrm{s}, \omega_{0}=10 \mathrm{rad} / \mathrm{s}$ (corresponding to $1.6 \mathrm{~Hz}$ ), $\tau^{*}=r_{\mathrm{c}} / v_{\mathrm{s}}=0.016 \mathrm{~s}$. The dipole-moment amplitude coefficient is then $m_{c}=100 \mathrm{~A} \cdot \mathrm{m}^{2}$. The calculated dipole-moment histories [as $F_{0}(t)$ ] are shown in Fig. 5-3, for the several $\gamma$ values. $F_{0}$ is not intrinsically normalized; its maximum value is $F_{\mathrm{m}} \approx 6$, so that $m_{0} \approx 600 \mathrm{~A} \cdot \mathrm{m}^{2}$. It is little affected by the choice of damping coefficient $\gamma$. This is a much weaker and slower source, as compared to the magnetic bubble. 
At late times, this dipole-moment calculation extends into geologic domains that, in practice, seriously depart from the assumed uniform, infinite, ideal case. The decrement truncation (as $e^{-\gamma t^{*}}$ ) is thus essential to a practical cutoff, and it is also authenticated by the remote MERLIN ground-motion data. (Note that $v_{\mathrm{s}} / \gamma \approx 600 \mathrm{~m}$, and $v_{\mathrm{s}} / \omega_{0} \approx 200 \mathrm{~m}$.) Hence, the surrogate magnetic dipole stands in for a relatively large current distribution; it is a valid source model for the Distant-sensor locations only $(\rho=5 \mathrm{~km})$. The Local sensors $(\rho=200 \mathrm{~m})$ are, in effect, immersed in the source.

\subsection{Sensor Signals}

In determining an upper-bound magnitude for the Local signals, I considered just one sensor location: near the South-surface intercept of the dipole axis, per Fig. 5-1. Prima facie, $\dot{r} \times B_{\mathrm{e}}$ appears to be very small so near the axis. However, the acoustic reflection has a large orthogonality, such that its vector product is almost at maximum value.

I addressed three possible Local-signal components:

- Dipole-moment field at very early times-before the acoustic wave reaches the sensor point.

- Gaussian approximation at peak motion-as the acoustic wave passes by the sensor point.

- Solenoidal field at late times-when the acoustic wave is well past the sensor point.

The second contribution overwhelms the other two (by $\times 100$ ). It derives from the Faradayinduction $E$-field through $\left[E \approx \vec{\lambda} \vec{B} \approx \mu_{0} H v_{\mathrm{s}}\right]=\left[\dot{r} B_{\mathrm{e}} \sin \psi=E_{\mathrm{m}} \sin \psi f(t)\right] . E_{\mathrm{m}}$ is the peak potential gradient: $E_{\mathrm{m}}=\left(B_{\mathrm{e}} \dot{r}_{\mathrm{c}}\right)\left(r_{\mathrm{c}} / s\right)^{10 / 3}$. [Now $\psi$ is specifically the Local angle between the Earth's magnetic field and the (reflected) particle-velocity vector; $s$ is the distance to the sensor point.]

Finally, the magnetic signal is

$\Delta H=H_{\mathrm{m}} \sin \psi f(t)$,

where $H_{\mathrm{m}}=E_{\mathrm{m}} / \mu_{0} v_{\mathrm{s}}$. Then $E_{\mathrm{m}} \approx 11 \mu \mathrm{V} / \mathrm{m}, H_{\mathrm{m}} \approx 0.0045 \mathrm{At} / \mathrm{m}$. The waveforms follow the acoustic signal, as shown in Fig. 5-2.

The Distant magnetic signal obtains from the dipole moment by the same "up-and-over" formalism as for the magnetic bubble. I can describe it via three orthogonal components of general form: $\Delta H_{i}=$ $\left(m_{\mathrm{c}} / 4 \pi \rho^{3}\right) G_{i}(\phi) F_{0}(t)$. $\left(G_{i}\right.$ is an angular function of the first order.) The peak magnitude coefficient is $H_{\mathrm{m}}=$ $\left(m_{0} / 4 \pi \rho^{3}\right) \approx 4 \times 10^{-10} \mathrm{At} / \mathrm{m}$. This is so far below "visibility" as to justifiably abandon further consideration. 


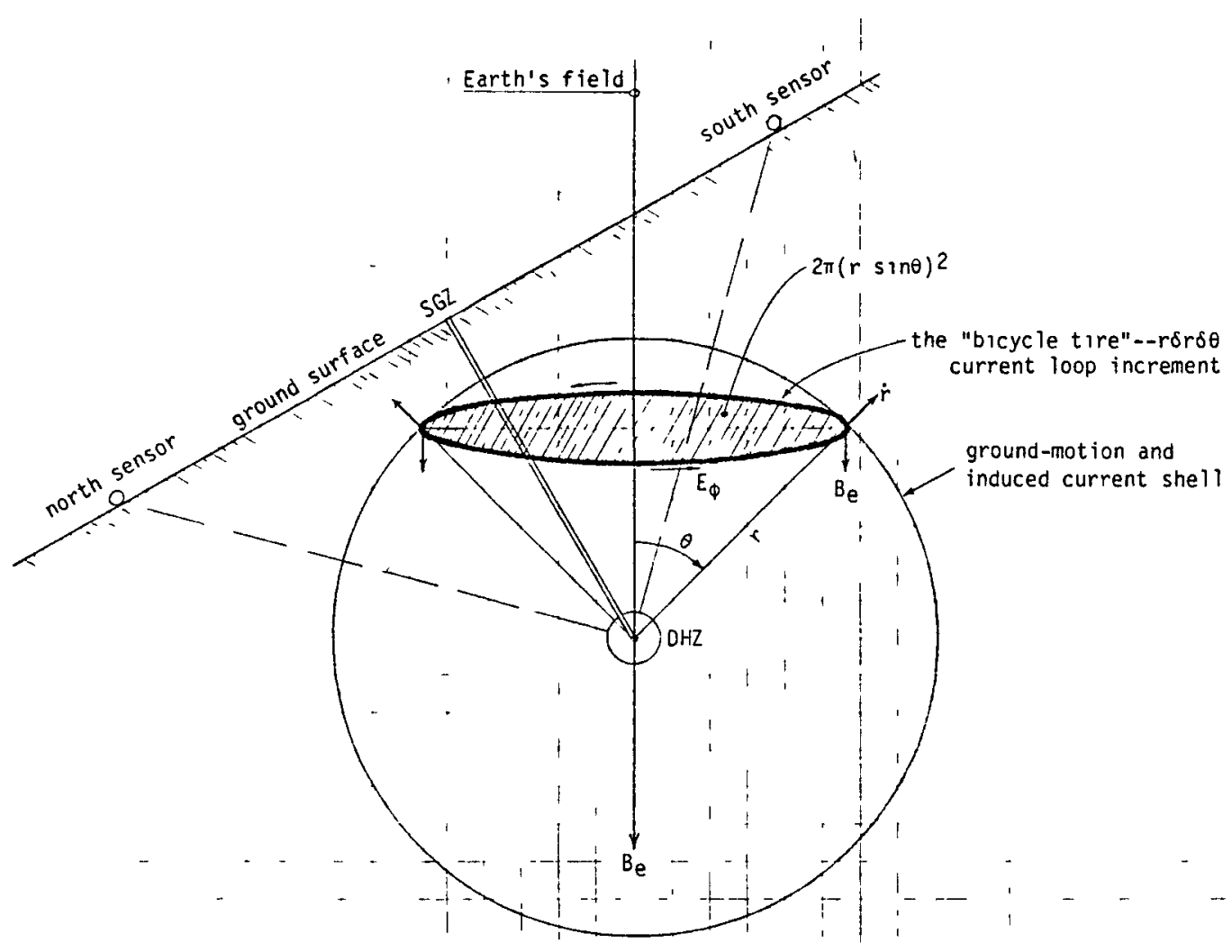

Figure 5-1. Ground-motion model-Earth's field frame. 


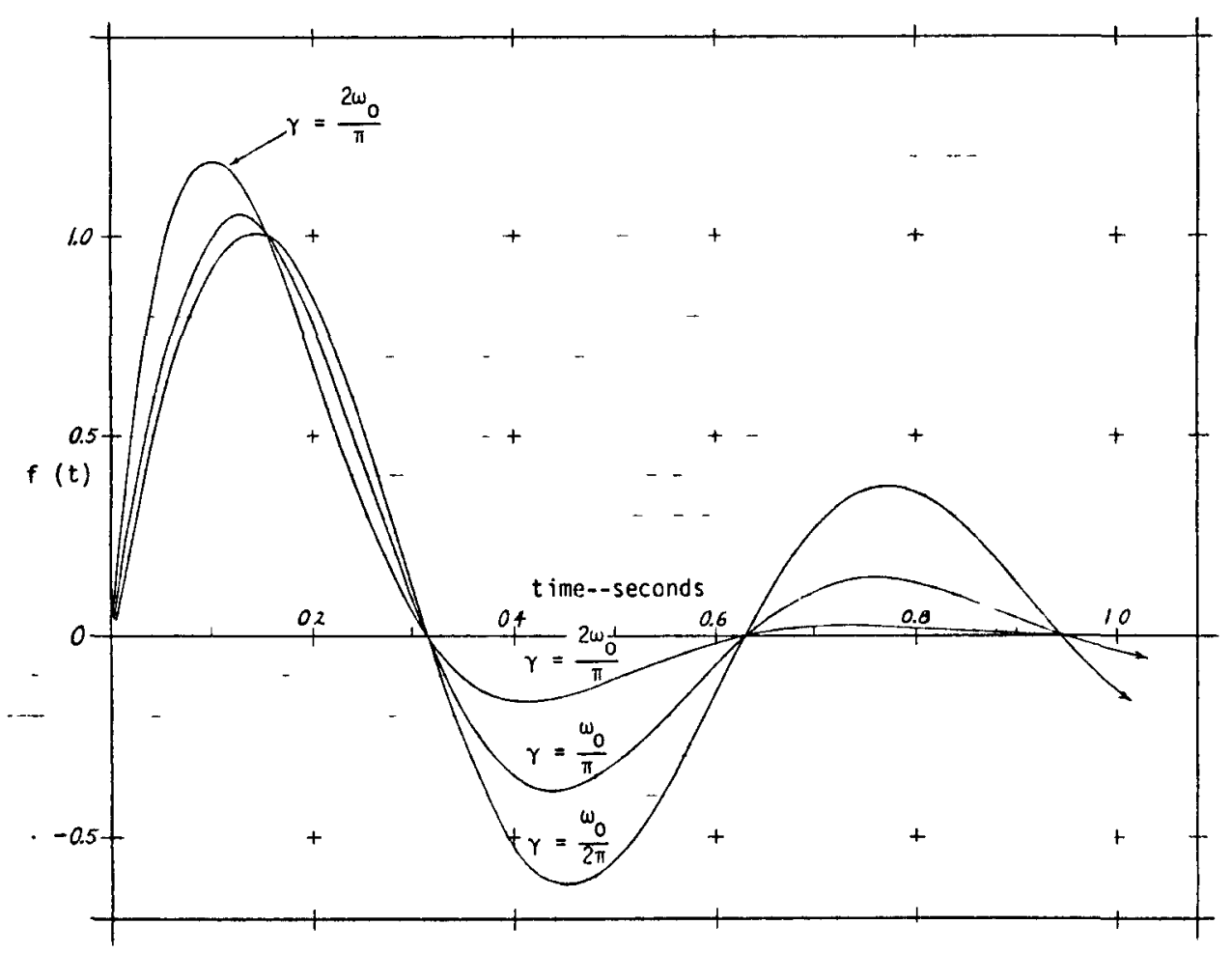

Figure 5-2. Assumed ground-motion function and "magneto-acoustic" waveform.

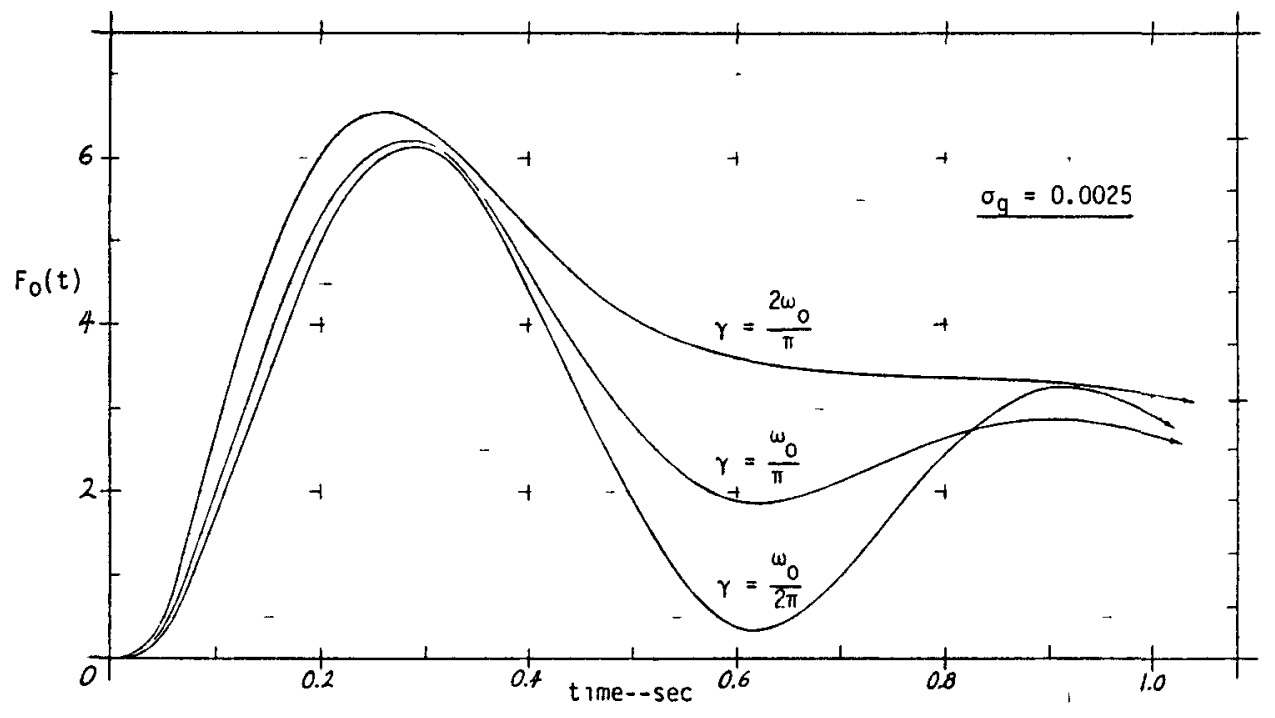

Figure 5-3. Ground-motion magnetic-dipole-moment history. 


\section{References}

1. W. Perret and R. C. Bass, Free-Field Ground Motion Free-Field Ground Motion Induced by Underground Explosions, Sandia National Laboratories, Albuquerque, N.M., SAND 74-0252 (1974).

2. S. Glasstone, The Effects of Nuclear Weapons, 3rd Ed. (U.S. GPO, Washington, D.C., 1974).

3. C. J. Zablocki, "Electrical Transients Observed During Underground Nuclear Explosions," J. Geophys. Res. 71(14), 3523 (1966).

4. M. B. Kraichman, Handbook of EM Propagation in Conducting Media, NAVMAT P-2302 (U.S. GPO, Washington, D.C., 1970).

5. E. D. Sundé, Earth Conduction Effects in Transmission Systems, (Dover Publications, New York, 1968).

6. Handbook of Mathematical Functions, National Bureau of Standards, Washington, D.C., AMS-55 (1972).

7. L. F. Wouters, Magnetic Field Effects from Underground Detonations, Lawrence Livermore National Laboratory, Livermore, Calif., UCID-4381 (1960).

8. W. R. Smythe, Static and Dynamic Electricity, 3rd Ed. (McGraw-Hill, New York, 1968), p. 351. 


\section{Appendix A \\ Compton-Dipole IFTs (Local Case Only)}

\section{Eigen Equations}

Phase:

$$
\left(\omega_{c} T\right) x_{m}^{2}-\sqrt{2} x_{m}-\left(m \phi_{m}+\psi_{m-1}\right)=0, \text { where } \phi_{m}=\phi_{m-1}+\delta \phi, \text { and } x_{m}^{2}=\omega_{m} / \omega_{\mathrm{c}} .
$$

Figenfrequencies:

$$
\begin{aligned}
x_{m}= & \frac{\sqrt{ } 2}{2\left(\omega_{\mathrm{c}} T\right)}\left[1+\sqrt{\left.1+2\left(\omega_{\mathrm{c}} T\right)\left(m \phi_{m}+\bar{\psi}_{-1}\right)\right] .}\right. \\
& \left\{\begin{array}{l}
\psi_{-1}^{\mathrm{e}}=\left[-\arctan \left(\frac{x^{2}}{4}\right)+\arctan (\sqrt{ } 2 x)\right]_{m-1} \\
\psi_{-1}^{\mathrm{h}}=\left[-\arctan \left(\frac{x^{2}}{4}\right)+\arctan \left(\frac{\sqrt{2} x}{1+\sqrt{2} x}\right)\right]_{m-1}
\end{array}\right\} \text { retrograde values }
\end{aligned}
$$

IFTs: $S_{\mathrm{e}^{\prime}} S_{\mathrm{h}}$ as in text.*

* Consolidated form not possible here (NB: casing-current II.T). 


\section{Appendix B \\ Casing-Current IFTs}

\section{Eigen Equations}

Phase:

$$
\left(\omega_{\mathrm{c}} T\right) x_{m}^{2}-R_{\phi} x_{m}-\left(m \theta_{m}+\xi_{m-1}\right)=0, \text { where } \theta_{m}=\theta_{m-1}+\delta \theta, \text { and } x_{m}^{2}=\omega_{m} / \omega_{\mathrm{c}} .
$$

Eigenfrequencies:

$$
\begin{aligned}
& x_{m}=\frac{R_{\phi}}{2\left(\omega_{c} T\right)}\left[1+\sqrt{1+\frac{4\left(\omega_{c} T\right)}{R_{\phi}^{2}}\left(m \theta_{m}+\xi_{m-1}\right)}\right] . \\
& \xi_{m}=\left[-\arctan \left(\frac{x^{2}}{5}\right)+\psi\right]_{m}
\end{aligned}
$$

IFTs (consolidated form): $S_{\text {eh }}$ as in text.

\section{Location Parameters*:}

$\psi, f(x)$ characterize $\mathrm{E}-\mathrm{H}$, and Local-Distant cases as follows:

Local fields $(\rho=200 \mathrm{~m}): R=\sqrt{ } 1.4, R_{\phi}=\sqrt{ } 2.4$

$$
\begin{array}{ll}
\psi^{\mathrm{e}}=\arctan R x & f^{\mathrm{e}}(x)=(1+R x) \sqrt{1+R^{2} x^{2}} \\
\psi^{\mathrm{h}}=\arctan \frac{\boldsymbol{R} x}{1+\boldsymbol{R} x} & f^{\mathrm{h}}(x)=\sqrt{1+2 R x+2 \boldsymbol{R}^{2} x^{2}}
\end{array}
$$

Distant fields $(\rho=5 \mathrm{~km}): R=\left[1-\left(4+x^{2}\right)^{-1 / 2}\right], R_{\phi}=1$

$$
\begin{array}{lll}
\psi^{\mathrm{e}}=\pi / 4 & f^{\mathrm{e}}(x)=x^{3} & f^{\mathrm{e}^{*}}=g^{-1} x^{5} \\
\psi^{\mathrm{h}}=0 & f^{\mathrm{h}}(x)=x^{2} & f^{\mathrm{h}^{*}}=g^{-1} x^{4}
\end{array}
$$

$f^{\mathrm{e}^{*}}$ and $f^{\mathrm{h}^{*}}$ identify the separate $\left(\gamma_{0} \rho\right)$ Fourier propagation terms in the Kraichman expressions, ${ }^{4}$ for which $\begin{array}{ll}\sigma_{\mathrm{g}} & =0.0025 \\ g & 3.75\end{array}$ 0.005 7.5 0.01 15

* These did not show up explicitly for the Compton dipole because all frequencies originated from the same place 
